University of Tennessee Health Science Center

UTHSC Digital Commons

\title{
The Heme-Regulated Inhibitor Pathway Modulates Susceptibility of Poor Prognosis B-Lineage Acute Leukemia to BH3-Mimetics
}

\author{
Kaitlyn Hill Smith \\ University of Tennessee Health Science Center
}

Follow this and additional works at: https://dc.uthsc.edu/dissertations

Part of the Hemic and Lymphatic Diseases Commons, Medical Cell Biology Commons, Medical Molecular Biology Commons, Neoplasms Commons, and the Oncology Commons

\section{Recommended Citation}

Smith, Kaitlyn Hill (https://orcid.org/0000-0002-9981-7883), "The Heme-Regulated Inhibitor Pathway Modulates Susceptibility of Poor Prognosis B-Lineage Acute Leukemia to BH3-Mimetics" (2021). Theses and Dissertations (ETD). Paper 550. http://dx.doi.org/10.21007/etd.cghs.2021.0529. 


\title{
The Heme-Regulated Inhibitor Pathway Modulates Susceptibility of Poor Prognosis B-Lineage Acute Leukemia to BH3-Mimetics
}

\begin{abstract}
Anti-apoptotic MCL1 is one of the most frequently amplified genes in human cancers and its elevated expression confers resistance to many therapeutics including the BH3-mimetic agents ABT-199 and ABT-263. The anti-malarial, dihydroartemisinin (DHA) translationally represses MCL-1 and synergizes with BH3-mimetics. To explore how DHA represses MCL-1, a genome-wide CRISPR screen identified that loss of genes in the heme synthesis pathway renders mouse BCR-ABL+B-ALL cells resistant to DHA-induced death. Mechanistically, DHA disrupts the interaction between heme and the elF2 $\alpha$ kinase heme regulated inhibitor (HRI) triggering the integrated stress response. Genetic ablation of Eif2ak1, which encodes HRI, blocks MCL-1 repression in response to DHA treatment and represses the synergistic killing of DHA and BH3-mimetics compared to wild-type leukemia. Furthermore, BTdCPU, a small-molecule activator of HRI, similarly triggers MCL-1 repression and synergizes with BH3-mimetics in mouse and human leukemia including both $\mathrm{Ph}+$ and $\mathrm{Ph}$-like B-ALL. Lastly, combinatorial treatment of leukemia bearing mice with both BTdCPU and a BH3-mimetic extended survival and repressed MCL-1 in vivo. These findings reveal that the HRI-dependent cellular heme-sensing pathway can modulate apoptosis in leukemic cells by repressing MCL-1 and increasing their responsiveness to BH3-mimetics. This signaling pathway could represent a generalizable mechanism for repressing MCL-1 expression in malignant cells and sensitizing them to available therapeutics.
\end{abstract}

\section{Document Type}

Dissertation

\section{Degree Name}

Doctor of Philosophy (PhD)

\section{Program}

Biomedical Sciences

Research Advisor

Joseph T. Opferman, Ph.D

Keywords

BH3-mimetics; HRI; MCL-1

\section{Subject Categories}

Diseases | Hemic and Lymphatic Diseases | Medical Cell Biology | Medical Molecular Biology | Medical Sciences | Medical Specialties | Medicine and Health Sciences | Neoplasms | Oncology 


\section{UNIVERSITY OF TENNESSEE HEALTH SCIENCE CENTER}

DOCTORAL DISSERTATION

\section{The Heme-Regulated Inhibitor Pathway Modulates Susceptibility of Poor Prognosis B-Lineage Acute Leukemia to BH3-Mimetics}

Author:

Kaitlyn Hill Smith
Advisor:

Joseph T. Opferman, Ph. D.

A Dissertation Presented for The Graduate Studies Council of

The University of Tennessee Health Science Center

in Partial Fulfillment of the Requirements for the Doctor of Philosophy degree from The University of Tennessee

in

Biomedical Sciences Program: Microbiology, Immunology, \& Biochemistry Track College of Graduate Health Sciences

March 2021 
Chapters 2 and 3 @ 2020 by American Association for Cancer Research All other material $\odot 2021$ by Kaitlyn Hill Smith.

\author{
All rights reserved.
}




\section{ACKNOWLEDGEMENTS}

I would like to acknowledge those who have helped and supported me throughout my graduate studies. I would first like to thank my advisor, Dr. Joseph Opferman. His guidance, patience, encouragement, and positive attitude have made my time in the lab a positive and productive experience. I am thankful that he has challenged me to think critically about experiments, come up with new ideas, and generate reasonable hypotheses. His mentorship and support have helped make me a better scientist. I would also like to thank all of the members of the Opferman lab; they have all supported my studies and research project, and given me advice throughout my time in the lab. I would especially like to thank Dr. Amit Budhraja and Dr. Meghan Turnis McGehee for their support and mentorship; they have taught me many techniques that have helped me complete various experiments and taught me how to properly present my data.

Additionally, the many scientific discussions Amit and I have had through the years have helped teach me how to think about science and experimental design.

I would like to thank my committee members, Dr. Shondra Miller, Dr. David Nelson, Dr. Stacey Ogden, and Dr. Gerard Zambetti. They have all supported me and given me advice throughout my project. They have asked questions which challenged me to think critically about parts of my project that I may have not originally focused on. Their advice and support have helped me grow as a scientist.

I would also like to thank my collaborators Dr. John Schuetz and Dr. John Lynch for their assistance with various experiments and helpful discussions. Additionally, I would like to thank the members of the Center for Advanced Genome Engineering, especially Dr. Patrick Connelly, for their direction and assistance with CRISPR technologies.

Finally, I would like to thank my family for their love and support throughout my time in graduate school. My husband, Chase, has supported me through the times of studying for tests, going to the lab super early or staying super late to finish experiments, stressing because an experiment didn't work, publishing my first first-author paper, and everything in-between. His constant encouragement, support, and fun-loving attitude have helped make my graduate school experience an enjoyable one. I would also like to thank my parents for their love, support, and encouragement through all of my years in school and for teaching me the importance of education. 


\begin{abstract}
Anti-apoptotic MCL1 is one of the most frequently amplified genes in human cancers and its elevated expression confers resistance to many therapeutics including the BH3-mimetic agents ABT-199 and ABT-263. The anti-malarial, dihydroartemisinin (DHA) translationally represses MCL-1 and synergizes with BH3-mimetics. To explore how DHA represses MCL-1, a genome-wide CRISPR screen identified that loss of genes in the heme synthesis pathway renders mouse $\mathrm{BCR}-\mathrm{ABL}^{+} \mathrm{B}-\mathrm{ALL}$ cells resistant to DHAinduced death. Mechanistically, DHA disrupts the interaction between heme and the eIF2 $\alpha$ kinase heme regulated inhibitor (HRI) triggering the integrated stress response. Genetic ablation of Eif2ak1, which encodes HRI, blocks MCL-1 repression in response to DHA treatment and represses the synergistic killing of DHA and BH3-mimetics compared to wild-type leukemia. Furthermore, BTdCPU, a small-molecule activator of HRI, similarly triggers MCL-1 repression and synergizes with BH3-mimetics in mouse and human leukemia including both $\mathrm{Ph}^{+}$and $\mathrm{Ph}$-like B-ALL. Lastly, combinatorial treatment of leukemia bearing mice with both BTdCPU and a BH3-mimetic extended survival and repressed MCL-1 in vivo. These findings reveal that the HRI-dependent cellular heme-sensing pathway can modulate apoptosis in leukemic cells by repressing MCL-1 and increasing their responsiveness to BH3-mimetics. This signaling pathway could represent a generalizable mechanism for repressing MCL-1 expression in malignant cells and sensitizing them to available therapeutics.
\end{abstract}




\section{TABLE OF CONTENTS}

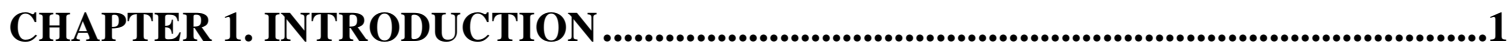

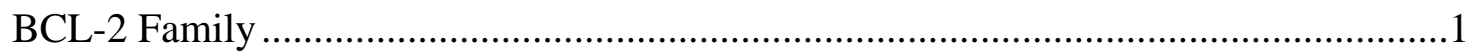

BCL-2 Family in Cancer................................................................................

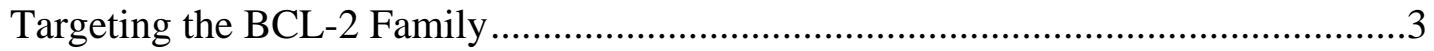

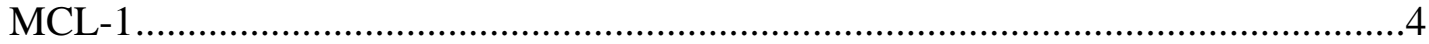

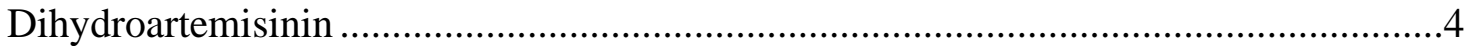

DHA as an Anti-Malarial Drug........................................................................

DHA and Anti-Cancer Activity ........................................................................

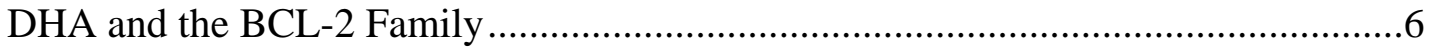

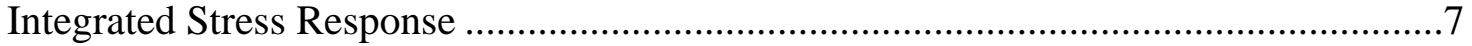

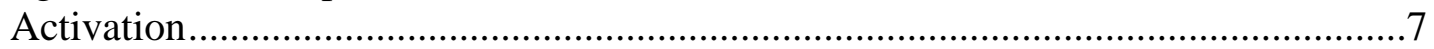

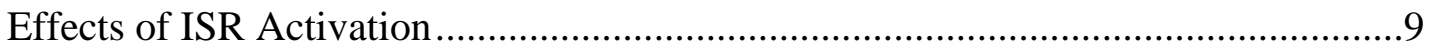

B-Lineage Acute Lymphoblastic Leukemia .............................................................. 11

Philadelphia Chromosome Positive B-ALL ....................................................... 11

Philadelphia Chromosome-Like B-ALL ..........................................................12

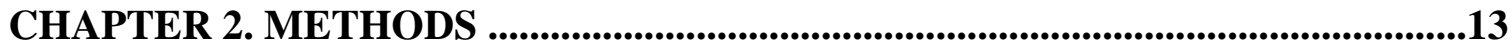

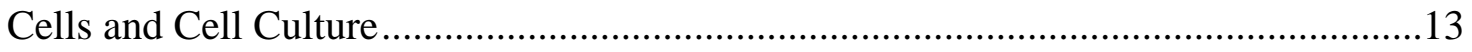

Genome-Wide CRISPR Screen ......................................................................... 13

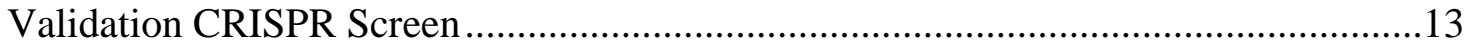

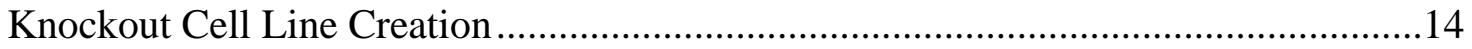

Immunoblotting and Antibodies ........................................................................ 14

Measuring Interaction Between HRI and Heme ...................................................14

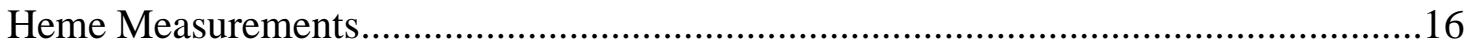

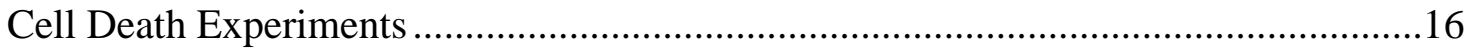

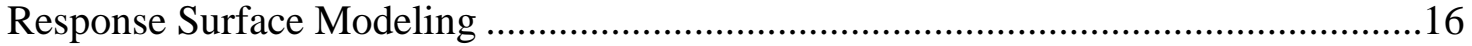

Patient-Derived Xenograft (PDX) Leukemia ........................................................ 17

Treatment of Leukemia in Recipient Mice .................................................................. 17

Pathology and Immunohistochemistry ......................................................... 17

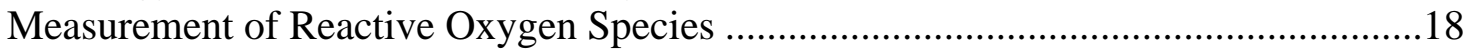

CHAPTER 3. RESULTS......................................................................................................19

CRISPR Screen Identifies Pathways Required for DHA-Induced Apoptosis ...............19

DHA Induces an HRI-Mediated eIF2 $\alpha$ Phosphorylation and MCL-1 Repression ........24

HRI Is Required for the Synergistic Response of DHA and BH3-Mimetics ...............28

Direct Activation of HRI Represses MCL-1 and Synergizes with BH3-Mimetics .......28

HRI Activation Synergizes with BH3-Mimetics in ALL Cell Lines............................32

HRI Activation Synergizes with BH3-Mimetics in Primary Patient-Derived

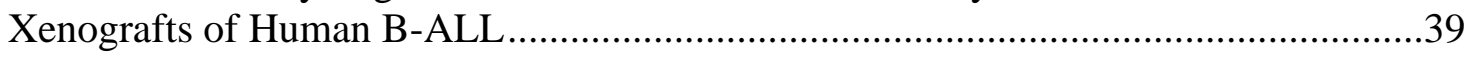

HRI Activation Represses MCL-1 and Synergizes with BH3-Mimetics in vivo ..........39 


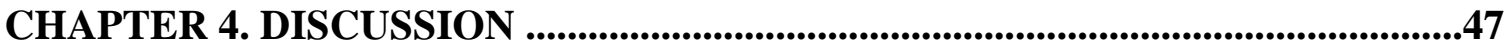

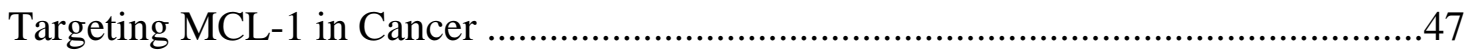

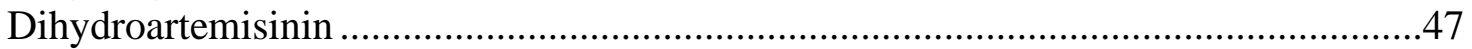

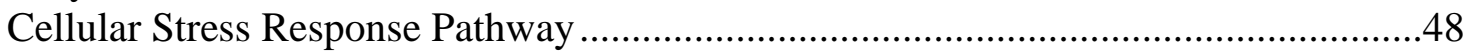

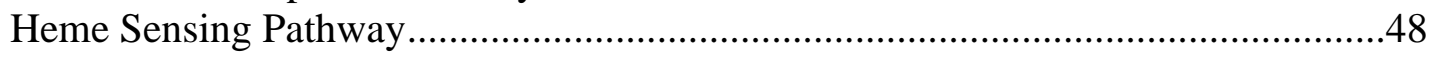

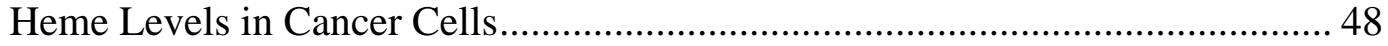

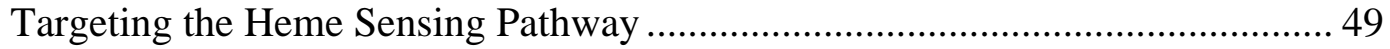

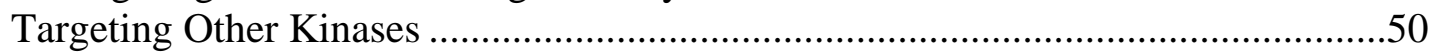

Potential for Combinatorial Uses.........................................................................50

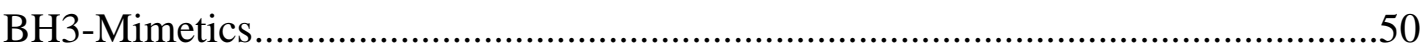

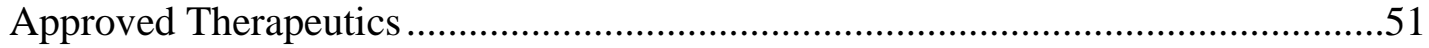

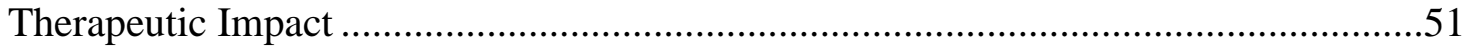

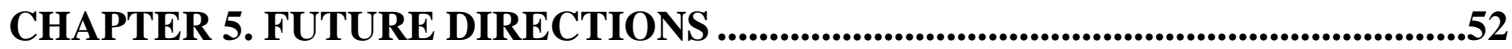

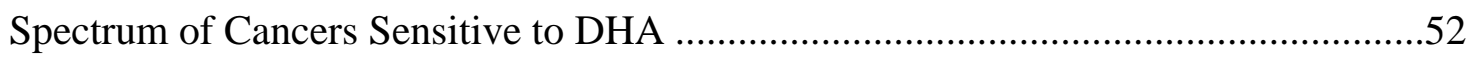

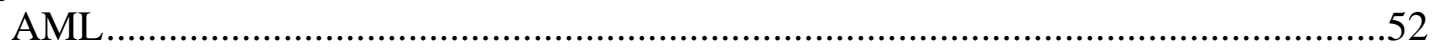

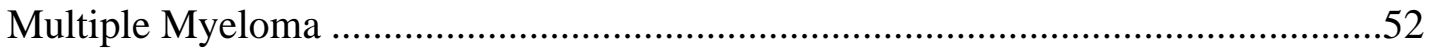

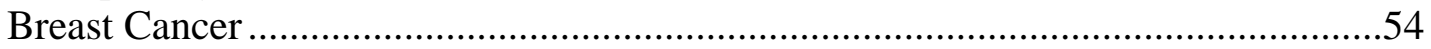

The Separation of ISR Activation and Apoptosis ..............................................54

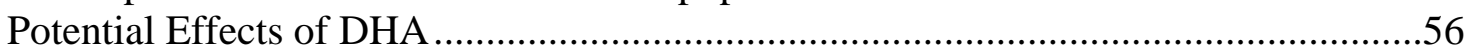

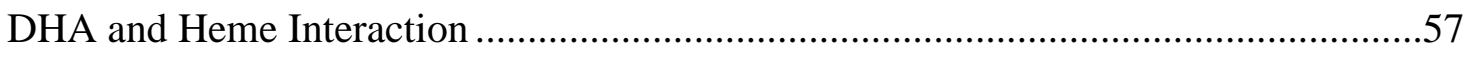

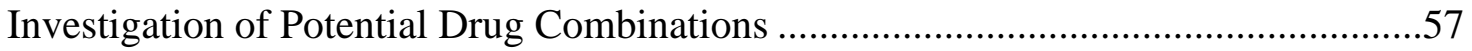

LIST OF REFERENCES ........................................................................................59

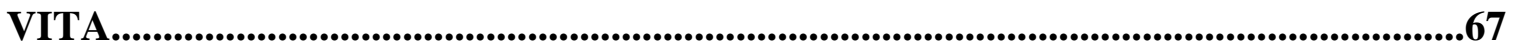




\section{LIST OF TABLES}

Table 2-1. $\quad$ sgRNA sequences for knockout cell lines .............................................15

Table 3-1. Top 35 hits from genome wide CRISPR screen, determined by

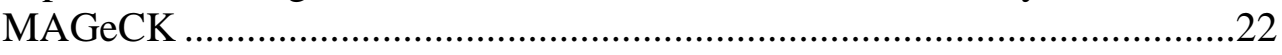




\section{LIST OF FIGURES}

Figure 1-1. Regulation of apoptosis by the BCL-2 family ........................................2

Figure 1-2. Integrated stress response signaling ...............................................

Figure 1-3. A model of the activation of HRI by multiple autophosphorylation in

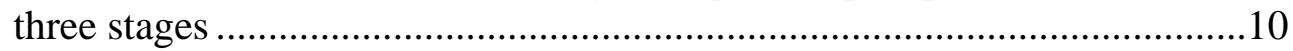

Figure 3-1. Stress pathways in cellular response to DHA ..........................................20

Figure 3-2. CRISPR screen identifies the requirement of the heme synthesis pathway for DHA-induced apoptosis ...................................................21

Figure 3-3. Control steps in heme metabolism.........................................................23

Figure 3-4. Effects of heme synthesis alterations on cellular response to DHA ...........25

Figure 3-5. HRI is required for DHA-induced MCL-1 repression ..............................26

Figure 3-6. HRI is required for the synergistic response of DHA and BH3-mimetics ...29

Figure 3-7. Direct activation of HRI represses MCL-1 and synergizes with BH3mimetics

Figure 3-8. HRI activation represses MCL-1 in human $\mathrm{Ph}^{+} \mathrm{BV}-173$ cells ....................33

Figure 3-9. HRI activation synergizes with $\mathrm{BH} 3$-mimetics in human $\mathrm{Ph}^{+} \mathrm{BV}-173$

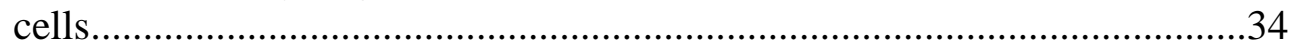

Figure 3-10. HRI activation synergizes with $\mathrm{BH} 3$-mimetics in human $\mathrm{Ph}^{+}$cell lines ......36

Figure 3-11. HRI activation represses MCL-1 and induces the integrated stress response in Ph-like ALL cell lines .........................................................

Figure 3-12. HRI activation synergizes with BH3-mimetics in Ph-like cell lines ...........40

Figure 3-13. HRI activation synergizes with BH3-mimetics and represses MCL-1 in PDX ALL cells

Figure 3-14. HRI activation represses MCL-1 and synergizes with BH3-mimetics in vivo

Figure 3-15. Cells do not acquire resistance to HRI activation combined with BH3mimetics in vivo

Figure 5-1. DHA represses MCL-1 in a panel of human AML cell lines .53 
Figure 5-2. DHA-induced apoptosis is ROS independent .........................................55 


\section{LIST OF ABBREVIATIONS}

\begin{tabular}{ll}
$\mu$ Ci/ml & Microcurie per milliliter \\
$\mu$ M & Micromolar \\
A1 & BCL-2 related protein A1 \\
ABL1 & Tyrosine-protein kinase ABL1 \\
ABL2 & Tyrosine-protein kinase ABL2 \\
ALA & Aminolevulinic acid \\
ALAD & Delta-aminolevulinic acid dehydratase \\
ALK & Anaplastic lymphoma receptor tyrosine kinase \\
AML & Acute myelogenous leukemia \\
ANOVA & Analysis of variance \\
APAF1 & Apoptotic protease-activating factor 1 \\
APC & Allophycocyanin \\
Arf & Cyclin dependent kinase inhibitor 2A \\
ATF4 & Activating transcription factor 4 \\
BAD & BCL-2 antagonist of cell death \\
BAK & BCL-2 antagonist/killer \\
B-ALL & B-cell acute lymphoblastic leukemia \\
BAX & BCL-2-associated protein \\
BCL-2 & B cell lymphoma 2 \\
BCL-W & BCL-2-like protein 2 \\
BCL-xL & BCL-2-like protein 1 \\
BCR-ABL & Breakpoint cluster region-Abelson tyrosine-protein kinase 1 \\
BH domain(s) & Bcl-2 homology domains \\
BID & BH3-interacting-domian death agonist \\
BIK & BCL2 Interacting Killer \\
BIM & BCL-2-interacting mediator of cell death \\
BiP/ GRP78 & Binding immunoglobulin protein \\
BMF & Bc12 modifying factor \\
bp & Basepairs \\
BRAF & B-rapidly accelerated fibrosarcoma \\
BTdCPU & 1-(benzo[d][1,2,3]thiadiazol-6-yl)-3-(3,4-dichlorophenyl)urea \\
C & Control \\
CDK4 & Cyclin dependent kinase 4 \\
CDK9 & Cyclin dependent kinase 9 \\
CHOP & C/EBP homologous protein \\
CHX & Cycloheximide \\
CKIT & Tyrosine-protein kinase KIT \\
CLL & Chronic lymphocytic leukemia \\
CML & Chronic myelogenous leukemia \\
CPOX & Coproporphyrinogen oxidase \\
CRISPR & Clustered regularly interspaced short palindromic repeats \\
CRLF2 & Cytokine receptor like factor 2 \\
DCFH-DA & Dichloro-dihydro-fluorescein diacetate \\
& \\
\hline
\end{tabular}




\begin{tabular}{ll} 
DFO & deferoxamine \\
DHA & Dihydroartemisinin \\
DHE & Dihydroethidium \\
DMSO & Dimethyl sulfoxide \\
DNA & Deoxyribonucleic acid \\
DP & DHA combined with piperaquine \\
DPI & Diphenyleneiodonium \\
EBF1-PDGFRB & Early B-cell factor 1-platelet-derived growth factor receptor- $\beta$ \\
EDB & Enzyme dilution buffer \\
EDTA & Ethylenediaminetetraacetic acid \\
EGFR & Epidermal growth factor receptor \\
eIF2B & Eukaryotic translation initiation factor 2B \\
eIF2 $\alpha$ & Eukaryotic translation initiation factor 2 $\alpha$ \\
ER & Endoplasmic reticulum \\
FDA & U.S. food and drug administration \\
FDR & False discovery rate \\
FECH & Ferrochelatase \\
FISH & Fluorescence in situ hybridization \\
GADD34 & Growth arrest and DNA damage-inducible protein \\
GCN2 & General control nonderepressible 2 \\
GDP & Guanosine diphosphate \\
GFP & Green fluorescent protein \\
GPx4 & Glutathione peroxidase 4 \\
GTP & Guanosine triphosphate \\
H\&E & Hematoxylin and eosin \\
HCl & Hydrochloric acid \\
HMBS & Hydroxymethylbilane synthase \\
HPLC & High-performance liquid chromatography \\
HRI & Heme-regulated inhibitor \\
HRK & Harakiri, BCL2 interacting protein \\
HRP & Horseradish peroxidase \\
IRE1 & Inositol-requiring enzyme 1 \\
ISR & Integrated stress response \\
ISRIB & Integrated stress response inhibitor \\
JAK2 & Janus kinase 2 \\
JAK-STAT & Janus kinase-signal transducer and activator of transcription \\
KO & Knock-out \\
MAGeCK & Model-based Analysis of genome-wide CRISPR-cas9 knockout \\
MCL-1 & Myeloid cell leukemia 1 \\
MET & MET proto-oncogene, receptor tyrosine kinase \\
Met-tRNA & Methionine transfer RNA \\
mg/kg & Milligrams per kilogram \\
mM & Millimolar \\
MnTBAP & Mn(III) tetrakis (4-benzoic acid) porphyrin \\
MnTE-2-PyP & Manganese (III) meso-tetrakis N-ethylpyridinium-2-yl porphyrin \\
MnTMPYP & Manganese(III) tetrakis(1-methyl-4-pyridyl)porphyrin \\
\hline &
\end{tabular}




\begin{tabular}{|c|c|}
\hline MOI & Multiplicity of infection \\
\hline MOMP & Mitochondrial outer membrane permeabilization \\
\hline NAC & $\mathrm{N}$-acetyl cysteine \\
\hline $\mathrm{NF}-\kappa \mathrm{B}$ & Nuclear factor $-\kappa B$ \\
\hline NGS & Next-generation sequencing \\
\hline $\mathrm{nM}$ & Nanomolar \\
\hline NOXA & Phorbol-12-myristate-13-acetate-induced protein 1 \\
\hline $\mathrm{OE}$ & Over-expressing \\
\hline PAX5 & Paired box 5 \\
\hline PAX5-JAK2 & Paired box 5-janus kinase 2 \\
\hline PCR & Polymerase chain reaction \\
\hline PDGFRB & Platelet-derived growth factor receptor \\
\hline PDX & Patient-derived xenograft \\
\hline P-eIF $2 \alpha$ & Phosphorylated eukaryotic translation initiation factor $2 \alpha$ \\
\hline PERK & PKR-like ER kinase \\
\hline $\mathrm{Ph}+$ & Philadelphia chromosome positive \\
\hline Ph-like & Philadelphia chromosome-like \\
\hline $\mathrm{PI} 3 \mathrm{~K} / \mathrm{AKT}$ & phosphoinositide 3-kinase/Protein kinase B \\
\hline PKR & Protein kinase RNA-activated \\
\hline pmol & Picomolar \\
\hline Pos $\mid \mathrm{fdr}$ & Positive false discovery rate \\
\hline Pos | lfc & Positive log fold change \\
\hline Pos $\mid \mathrm{p}$-value & Positive $\mathrm{p}$-value \\
\hline Pos | rank & Positive rank \\
\hline Pos $\mid$ Score & Positive score \\
\hline PPOX & Protoporphyrinogen oxidase \\
\hline PUMA & p53 upregulated modulator of apoptosis \\
\hline Ras & Rat sarcoma \\
\hline Ras-MAPK & Rat sarcoma mitogen-activated protein kinase \\
\hline RCSD1-AB1 & RCSD domain containing 1-Abelson tyrosine-protein kinase 1 \\
\hline RCSD1-ABL2 & RCSD domain containing 1-Abelson tyrosine-protein kinase 2 \\
\hline RNA & Ribonucleic acid \\
\hline RNP & Ribonuclear proteins \\
\hline ROS & Reactive oxygen species \\
\hline RT-PCR & Real time polymerase chain reaction \\
\hline SA & Succinylacetone \\
\hline SEM & Standard error of the mean \\
\hline sgRNA & Single guide RNA \\
\hline siRNA & Small interfering RNA \\
\hline SJCRH & St. Jude Children's Research Hospital \\
\hline SJCRHACUC & $\begin{array}{l}\text { St. Jude Children's Research Hospital animal care and use } \\
\text { committee }\end{array}$ \\
\hline SLL & Small lymphocytic lymphoma \\
\hline TEM & Transmission electron microscopy \\
\hline Thp & Thapsigargin \\
\hline TKI & Tyrosine kinase inhibitor \\
\hline
\end{tabular}


TM

UROD

UROS

WBC

WT
Transmembrane

Uroporphyrinogen decarboxylase

Uroporphyrinogen III synthase

White blood cells

Wild-type 


\section{CHAPTER 1. INTRODUCTION}

\section{BCL-2 Family}

The BCL-2 family of proteins regulates the intrinsic pathway of apoptosis through a balance of pro- and anti- apoptotic members [1]. In the presence of a cellular stress the pro-apoptotic BH3-only proteins, such as NOXA, PUMA, or BIM, are activated. These proteins then bind to specific anti-apoptotic proteins, such as MCL-1, BCL-2, or BCL-xL [1]. The inhibition of the anti-apoptotics by the BH3-only pro-apoptotics allows for the pro-apoptotic effectors, such as BAX and BAK, to become activated. Upon activation, $\mathrm{BAX}$ and BAK oligomerize and generate pores in the outer mitochondrial membrane (known as the point of no return), allowing for cytochrome $c$ release (Figure 1-1B) [2] . Cytochrome $c$, along with APAF1 and Caspase 9, then form the apoptosome. The apoptosome activates procaspase 3; the activation of these caspases then leads to proteolysis and eventual cell death [1].

All members of the BCL-2 family contain BCL-2 homology (BH) domain(s). The anti-apoptotic members (BCL-2, BCL-xL, MCL-1, BCL-W and A1) and the proapoptotic effectors (BAX, BAK, and BOK) share BH1-4 domains. The pro-apoptotic BH3-only proteins (NOXA, PUMA, BIM, BID, BAD, BMF, HRK, and BIK), contain only the BH3 domain (Figure 1-1A) [2]. The pro-apoptotic proteins bind to antiapoptotic proteins with different affinities [3] . For example, NOXA binds only to MCL1, but BIM, BID, and PUMA, can bind to all the anti-apoptotics [3] . These domains and interactions are important when it comes to the role the BCL-2 family plays in cancer and has informed how we can target this family, especially when it comes to drug design to target specific proteins [4].

\section{BCL-2 Family in Cancer}

The BCL-2 family is known to play a role in many types of cancer, including both solid tumors and hematological malignancies [5]. The resistance to cell death is one of the hallmarks of cancer as described by Hanahan and Weinberg []], so as one can imagine, misregulation of the BCL-2 family can lead to the development of cancer. In fact, the association of members of the BCL-2 family and cancer dates back as far as the 1980s $[\underline{7}, \underline{8}]$. The upregulation of anti-apoptotic proteins can occur through multiple mechanisms including translocations [9], gene amplifications [5], and increased gene translation [10] all of which can lead to the initiation or survival of cancer cells.

Another important role the BCL-2 family plays in cancer is in the sensitivity to cancer therapeutics. Montero, et al. showed that silencing MCL1 sensitized a variety of cancer cell lines to their respective targeted chemotherapeutic, including BRAF, CKIT, EGFR, MET, and ALK inhibitors [11]. This group went on to show that this was because these drugs lead to the destabilization of NOXA mRNA, which generates a dependence on MCL-1 [11]. This highlights the way in which interactions between 
A.

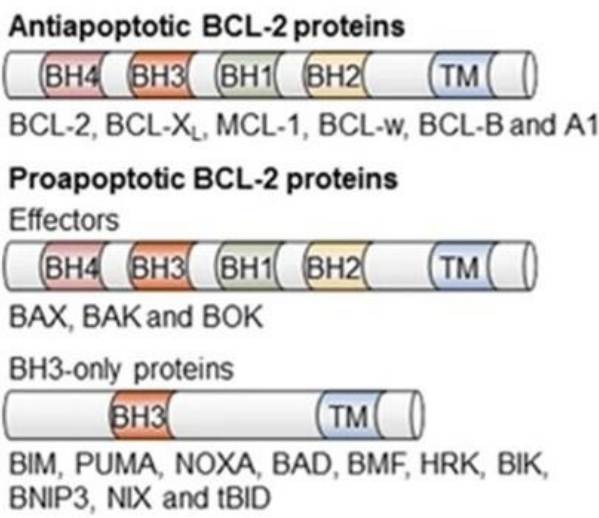

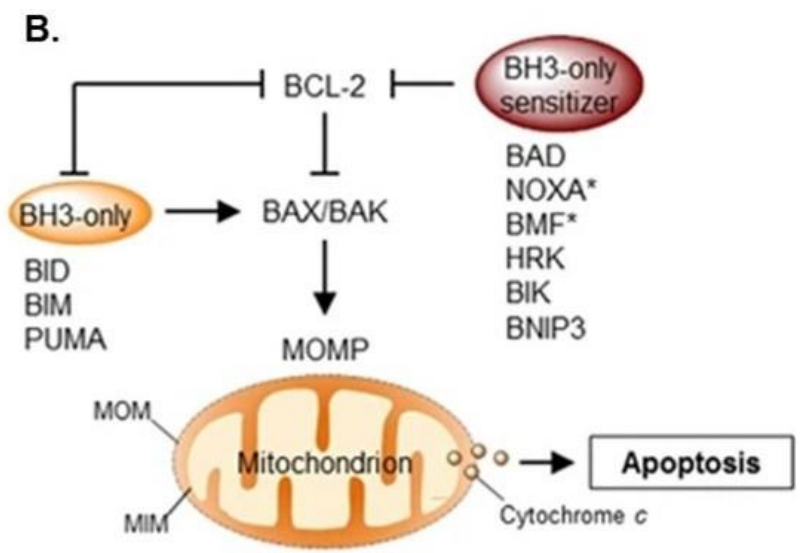

\section{Figure 1-1. Regulation of apoptosis by the BCL-2 family}

(A) The BCL-2 family of proteins is divided into three groups based on their functional role in the regulation of apoptosis and the number of BCL-2 homology $(\mathrm{BH})$ domains they bear. (B) The BCL-2 proteins regulate the permeabilization of the mitochondrial outer membrane (MOMP) and apoptosis through a specific network of heterodimeric interactions. Reprinted with permission from Springer Nature. Pihan, P., A. CarrerasSureda, and C. Hetz, BCL-2 family: integrating stress responses at the ER to control cell demise. Cell Death Differ, 2017. 24(9): p. 1478-1487. 
members of this family are important in drug sensitivity. Other examples of the role of the BCL-2 family in drug resistance have been seen in neuroblastoma, where silencing MCL1 sensitized neuroblastoma cells to standard chemotherapeutics (etoposide and doxorubicin); and osteosarcoma, where suppressing MCL-1 sensitized cells to cisplatin and doxorubicin $[\underline{12}, \underline{13}]$. It is also known that MCL-1 can play a role in resistance to drugs aimed at targeting other members of the BCL-2 family [14]. Because the upregulation of anti-apoptotic proteins is known to be involved in both the initiation and survival of cancer cells, and that this protein family is involved in chemo resistance, there have been efforts to specifically target this pathway.

\section{Targeting the BCL-2 Family}

BH3-mimetics are a class of drugs designed to target the BCL-2 family. As the name suggests, they mimic the $\mathrm{BH} 3$-only proteins and bind and inhibit the anti-apoptotic proteins. The first BH3-mimetic developed was ABT-737 which targets BCL-2, BCL$\mathrm{XL}$, and BCL-W [15]; years later, this was further developed into ABT-263 (navitoclax) which targets the same proteins with improved pharmacokinetics [16]. ABT-263 was the first $\mathrm{BH} 3$-mimetic to reach the clinic; after promising pre-clinical results it was found that it induced a dose-limiting toxicity of thrombocytopenia due to the on-target inhibition of BCL-XL [17]. ABT-199 (venetoclax) was then developed to target BCL-2 specifically and was the first BH3-mimetic to gain FDA approval. Venetoclax was first approved for the treatment of adults with chronic lymphocytic leukemia (CLL) with a $17 p$ deletion, who had failed prior therapy in 2016. Venetoclax has since been approved for the treatment of CLL (with or without 17p deletion) and small lymphocytic lymphoma (SLL), and in combination with other chemotherapies in adults with acute myelogenous leukemia (AML) [18].

Development of MCL-1 inhibitors has been slower, partially because it is more challenging to target the surface features and it has a long shallow hydrophobic binding pocket [19]. The first selective MCL-1 inhibitor reported to have in vivo activity was S63845 in 2016 [20]. Some MCL-1 inhibitors, including S63845 (MIK665), AMG397, AMG176, and AZD5991 have reached clinical trials, for treatment of patients with various types of cancer including multiple myeloma, AML, non-Hodgkin's lymphoma, and diffuse large B-cell lymphoma [21, 22]. Although the pre-clinical data was encouraging the clinical trials have experienced some setbacks, as the trials on both Amgen inhibitors (AMG176, and AMG397) were placed on hold due to safety concerns of cardiac toxicity. Cardiac toxicity should not come as too much of a surprise due to the known role of MCL-1 in cardiomyocytes [23, 24]. The difficulty of MCL-1 specific inhibitor development coupled with the possibility of on-target toxicity highlights the need for alternative strategies to target this protein. 


\section{MCL-1}

Just as other members of the BCL-2 family are involved in cancer, MCL-1 is no exception. MCL1 is one of the most frequently amplified genes in human cancer [5]; highlighting the importance of mechanisms to target it. Although MCL-1 is most commonly known as an essential anti-apoptotic protein, it does have other functions aside from is role in apoptosis, which raises concern for possible toxicities associated with direct inhibition of the protein. As mentioned above, MCL-1 is known to be important in cardiomyocytes. Using a cardiac specific inducible $\mathrm{Mcll}$ deletion model, it was shown that deletion of $\mathrm{Mcll}$ in the adult mouse cardiac muscle induced fatal cardiomyopathy within weeks of deletion [23, 24]. Further, this need for Mcll in cardiomyocytes was not solely due to apoptotic functions; when Bax and Bak were deleted in combination with Mcll, although there was some rescue of the lethality, there were still mitochondrial abnormalities. These abnormalities included fragmented cristae structure as shown by TEM, and respiration defects as shown by the oxygen consumption rate.

MCL-1 is also known to be involved in various processes in different tissues. It is required for the survival of hematopoietic stem cells and deletion of $\mathrm{Mcll}$ results in the ablation of bone marrow [25]. MCL-1 has also been shown to be involved in various cell types of the immune system, including the survival of B and T lymphocytes [26], the survival of activated B cells and memory B cells [27], and the survival of natural killer cells [28]. Another important function of MCL-1 is seen in hepatocytes; the loss of Mcll in hepatocytes was shown to induce liver damage and resulted in smaller livers compared to littermate controls and fostered the formation of hepatocellular carcinomas [29]. These roles of $\mathrm{Mcll}$ in various cell types suggest a major barrier in the direct inhibition of MCL-1 due to possible heart, liver, hematopoietic, and immune complications.

Because the direct inhibition of MCL-1 may trigger unacceptable toxicities, it is important to find other ways of targeting this protein. One aspect of MCL-1 that is different than the other anti-apoptotic proteins is that it has a much shorter half-life. The half-life of MCL-1 is estimated to be around two hours where BCL-2, BCL-W, and BCLXL have half-lives of around 20 hours [30]. This suggests that a drug which could prevent the synthesis of MCL-1 would result in the rapid repression of the protein due to its rapid degradation. A number of studies have identified alternative strategies to target MCL-1 indirectly, one of which is through cyclin-dependent kinase 9 (CDK9) inhibition. CDK9 is a regulator of transcription, and it has been shown that the CDK9 inhibitor AZD4573 results in the rapid repression of MCL-1 and induction of apoptosis in a panel of cancer cell lines [31]. Another example of alternative ways to target MCL-1 includes the repression of translation by the anti-malarial drug dihydroartemisinin [32].

\section{Dihydroartemisinin}

Dihydroartemisinin (DHA) is an approved, well tolerated, artemisinin-based antimalarial drug [33]. Artemisinin is derived from an ancient Chinese herbal medicine; the sweet wormwood plant Artemisia annua was used as a Chinese herbal medicine for 
patients with fever dating back 2000 years [34]. It wasn't until the 1960s that scientists found this compound to have antimalarial activity [34]. Since then, many synthetic derivatives of artemisinin have been developed, including DHA, artesunate, and artemether.

\section{DHA as an Anti-Malarial Drug}

DHA is generally used as an anti-malarial drug in combination with piperaquine. DHA combined with piperaquine (DP) treatment is quite effective and well tolerated, with a cure rate of approximately $98 \%$, and only a few, mild side effects including gastrointestinal events, headache, or dizziness [33]. The dosing of DP is generally 2-10 $\mathrm{mg} / \mathrm{kg}$ of DHA with $16-27 \mathrm{mg} / \mathrm{kg}$ of piperaquine daily for 3 days [35]. The mechanism of DHA as an antimalarial involves the formation of free radicals. Artemisinins contain an endoperoxide bridge which is broken to generate the free radicals that are toxic to the malarial parasite. This is done through interactions with intraparasitic heme-iron which activates the artemisinin [36]. Artemisinins form a covalent interaction with heme which has been shown by the isolation of heme-artemisinin adducts [37]. Further, it was shown that artemisinin binds to hemoproteins, including hemoglobin, but does not react with heme-free globin [38]. These studies indicate that the presence of heme is required for DHA to act as an anti-malarial drug.

\section{DHA and Anti-Cancer Activity}

In addition to being an anti-malarial drug, DHA has also been shown to have anticancer activity in various cancers, and there have been a wide variety of mechanisms proposed for how DHA can trigger cancer cell killing [39]. One example of the anticancer activity of DHA can be seen in Philadelphia chromosome positive acute lymphoblastic leukemia. It was shown that DHA is potent against these leukemic cells, including those resistant to tyrosine kinase inhibitors; however, a mechanism of how DHA could target these cells was unclear, though it was speculated to involve a downregulation of c-MYC [40]. One commonly proposed mechanism is that DHA is toxic to cancer cells through the induction of reactive oxygen species (ROS). This mechanism fits with the way DHA works as an antimalarial because of the known production of free radicals. It has been shown that DHA can induce autophagy through the generation of ROS in CML (K562) cells [41]. This has also been shown in AML cells where DHA treatment induced ROS generation, measured by DCFH-DA staining [42]. Various other publications show that DHA can induce ROS generation in cancer cells including glioma cells [43], lung adenocarcinoma cells [44], and ovarian cancer cells [45]. Although DHA treatment can lead to the generation of ROS it is unclear if this is the only mechanism of toxicity because many studies indicate that this ROS generation is responsible for sensitization to other therapies, not that it alone leads to the death of cancer cells. For example, in the case of lung adenocarcinoma cells, the ROS generation by DHA sensitized the cells to arsenic trioxide, but had little effect on cell death on its own [44]. Additionally, there are studies which show that DHA induces apoptosis 
completely independent of ROS generation. DHA was shown to induce superoxide anion in promyelocytic leukemia cells; however, when the ROS induction was prevented by a superoxide anion scavenger (MnTE-2-PyP) there was no rescue of cell death, indicating that the death was independent of ROS generation [46]. These studies suggest that although DHA may induce ROS generation in cancer cells, it may not be the sole mechanism responsible for the DHA induced toxicity.

There have been other proposed mechanisms as to how artemisinin and its derivatives can target cancer cells. One of these is through the inhibition of angiogenesis; which was suggested to be through the suppression of vascular endothelial growth factor and angiopoietin-1 expression in multiple myeloma cells [47]. Another proposed mechanism of artemisinins is the induction of cell cycle arrest. An example of this was shown in endometrial cancer cells, where artemisinin inhibited cell proliferation by inducing a G1 cell cycle arrest through a repression of CDK4 gene expression by disruptions in NF- $\kappa \mathrm{B}$ signaling [뇨] . Another interesting theory of how DHA, or other artemisinins, can target cancer cells is through ferroptosis, which is the iron dependent cell death due to accumulation of lipid-based ROS [49]. This has been shown in head and neck carcinoma cells, where DHA induced cell death which was rescued by the addition of the iron chelator deferoxamine (DFO). DHA also reduced levels of GPx4 and Ras in these cells, which are believed to lead to the induction of ferroptosis when inactive [50]. Although this study did provide some evidence of the induction of ferroptosis, it did go on to show that ferroptosis may not be the sole mechanism of cell death induction and that apoptosis was also involved, as evidenced by the dependence on caspases.

\section{DHA and the BCL-2 Family}

In addition to the various proposed mechanisms of how DHA can act to induce apoptosis it can also induce alterations in the BCL-2 family in cancer cells. It was recently shown that DHA can translationally repress MCL-1 and induce apoptosis in $\mathrm{BCR}-\mathrm{ABL}^{+} \mathrm{B}-\mathrm{ALL}$ cells; the induction of apoptosis was proven by the rescue of cell death by deletion of Bax and Bak, or the inhibition of caspases [32]. The repression of MCL-1 is perhaps of even more importance and interest because of the known role MCL1 plays in a variety of cancer types. Here, the authors showed that MCL-1 is translationally repressed, as evidenced by the lack of change in mRNA, the substantial decrease in MCL-1 protein, the repression of global protein synthesis, and the lack of change in MCL-1 degradation time. Interestingly, none of the other anti-apoptotic BCL2 family members were repressed by DHA. The repression of MCL-1 by DHA was also shown to be highly synergistic with the BH3-mimetic ABT-263, which targets BCL-2, BCL-XL, and BCL-W. The synergy between DHA and ABT-263 was observed in a mouse derived cell line, human $\mathrm{Ph}^{+}$cell lines, and PDX cells. Additionally, MCL-1 was repressed in vivo by DHA in as short as $8 \mathrm{~h}$, and the combination of DHA and ABT-263 prolonged survival in leukemia bearing mice without any overt toxicities highlighting potential uses of DHA as a therapeutic. 
How, mechanistically, DHA triggers MCL-1 repression was unclear, but several possibilities were tested. One of these possibilities was that the MCL-1 repression was due to the induction of ROS. DHA did induce ROS in these cells; however, when the ROS was scavenged by either MnTMPyP or DPI there was no rescue of the MCL-1 repression, indicating that ROS production was not responsible for the DHA induced MCL-1 repression. By microarray analysis it was found that DHA induced a gene expression signature consistent with that of induction of ER stress. Again, the induction of stress response genes was shown to be independent of ROS induction. This study highlights a novel mechanism of how DHA could be used to target cancer cells through repression of a protein known to play a role in initiation and survival of many types of cancers; however, further work was needed to determine the mechanism triggering MCL1 repression in response to DHA.

\section{Integrated Stress Response}

\section{Activation}

The integrated stress response is a cellular stress response pathway that can be activated by a variety of stressors. This pathway leads to cellular responses, which aim to help the cell adapt and survive the stress; however, when the stress is too severe the pathway will induce signaling leading to cell death [51]. There are four known kinases that are the regulators of this pathway, each of which respond to distinct stressors (Figure 1-2). PKR-like ER kinase (PERK), is activated upon the induction of endoplasmic reticulum (ER) stress; double-stranded RNA-dependent protein kinase (PKR) is activated by viral infection; general control non-depressible protein 2 (GCN2) is activated by nutrient deprivation; and heme regulated inhibitor (HRI) is activated by cellular heme deprivation [51]. These are known to be the only four eIF2 $\alpha$ kinases; the deletion of all four at once led to a lack of induction of the integrated stress response pathway by a panel of stressors [52].

The PERK kinase is found in the ER lumen and is activated upon ER stress, due to the accumulation of unfolded proteins. In unstressed cells PERK is bound to the BiP ER chaperone protein (also known as GRP78) which keeps the kinase inactive. Upon ER stress, BiP dissociates from PERK, allowing it to oligomerize and autophosphorylate to become fully active [53]. The GCN2 kinase has mostly been studied in the context of budding yeast. When a cell is under amino-acid starvation conditions uncharged tRNAs accumulate and bind to GCN2; this causes a conformational change that leads to activation of the kinase [54]. The activation of PKR is in response to viral infection and is dependent on the presence of double stranded RNA. The dsRNA, which is believed to require a minimum of $30 \mathrm{bp}$, binds to two PKR monomers causing them to dimerize and autophosphorylate to become active [55].

The HRI kinase is generally activated in response to a cellular heme deprivation. This kinase has 2 heme binding sites, one in the N-terminal domain, which is a stable 


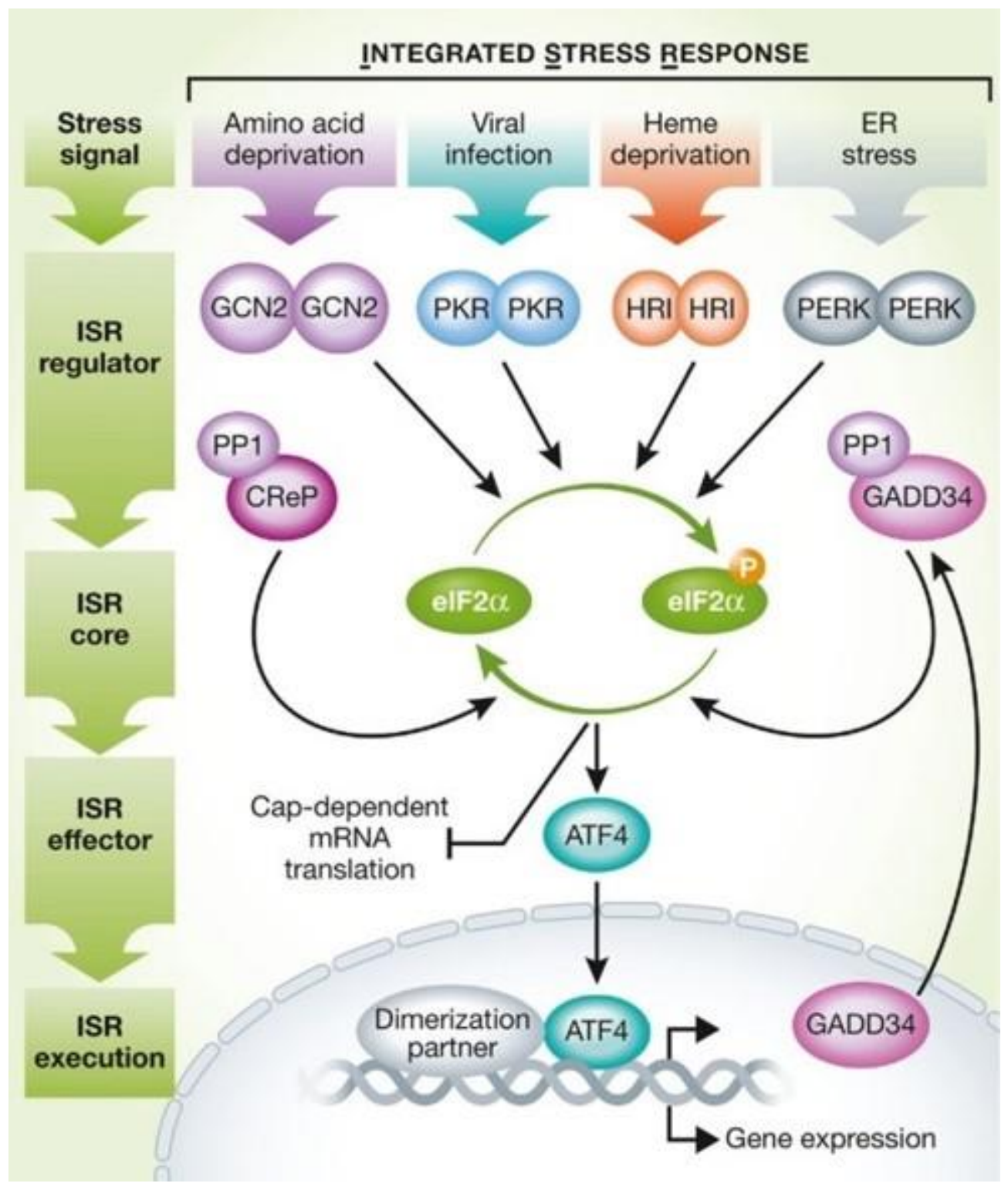

Figure 1-2. Integrated stress response signaling

ER stress, viral infection, and other cellular stress signals activate PERK, PKR, HRI, and GCN2 kinases that converge on phosphorylation of eIF2 $\alpha$, the core of ISR. This leads to global attenuation of Cap-dependent translation while concomitantly initiates the preferential translation of ISR-specific mRNAs, such as ATF4. ATF4 is the main effector of the ISR. Termination of the ISR is regulated by the constitutively expressed CReP and stress-inducible phosphatase GADD34 that dephosphorylate eIF2 $\alpha$. Arrows denote activation or induction, while blunt lines indicate inhibition. Reprinted with permission from John Wiley and Sons, Inc. Pakos-Zebrucka, K., et al., The integrated stress response. EMBO Rep, 2016. 17(10): p. 1374-1395. 
binding site, and one in the kinase insertion region, which is where heme regulates the kinase activity. HRI requires multiple autophosphorylation events to become fully activated. When HRI is synthesized, it dimerizes, binds heme at the stable N-terminus site, and undergoes two autophosphorylation steps. At this point, heme availability regulates the kinase activity of HRI. When present heme binds HRI in the kinase insertion region it leads to the formation of a disulfide bond, keeping the kinase inactive; this is a reversible process. When heme is unavailable, or the binding between heme and HRI is disrupted, HRI then undergoes a third autophosphorylation at threonine 485 resulting in the irreversible activation of the kinase (Figure 1-3) [ㄷ6, 57].

\section{Effects of ISR Activation}

Activation of any of the kinases involved in the ISR pathway leads to the phosphorylation of eIF2 $\alpha$; this phosphorylation leads to the inhibition of global capdependent protein translation (Figure 1-2). Generally, eIF $2 \alpha$ forms a complex with GTP and Met-tRNA which, along with other initiation factors, forms the $43 \mathrm{~S}$ pre-initiation complex and binds the 5' cap of mRNA. This allows for the recognition of initiation codon by the tRNA ${ }^{\text {Met; }}$; which leads to the GTP hydrolysis of eIF2 $\alpha$ and its dissociation from the $48 \mathrm{~S}$ complex. The eIF $2 \alpha$ can then be used to form another pre-initiation complex and begin a new round of translation; this process requires the guanine nucleotide exchange by eIF2B to convert eIF2-GDP to eIF2-GTP [58]. When the ISR pathway is active and eIF2 $\alpha$ is phosphorylated the exchange of GDP for GTP by eIF2B is blocked, preventing formation of the $43 \mathrm{~S}$ pre-initiation complex and ultimately global cap-dependent translation [51].

Although global protein translation is inhibited by the phosphorylation of eIF2 $\alpha$, this phosphorylation still leads to the translation of a small subset of mRNAs of stress response genes, such as ATF4 (Figure 1-2). When the cell is unstressed and eIF2 $\alpha$ is not phosphorylated, the ribosomes scan and initiate translation at an open reading frame that is out of frame with the ATF4 coding sequence, preventing its translation. Upon eIF2 $\alpha$ phosphorylation ribosomes recruit another ternary complex downstream of the AUG start codon that leads to the selective translation of ATF4 [59]. ATF4 then acts as a transcription factor, inducing stress response genes, such as DDIT3 (CHOP). Again, although global protein translation is shut down, these genes are translated by a capindependent mechanism of translation involving the recruitment of ribosomes to internal ribosome entry sites [우] .

The inhibition of translation for a short amount of time generally helps the cell survive the stress. For example, when heme levels are low in erythroid cells, the inhibition of translation until heme may become available, prevents the accumulation and aggregation of globin, which can result in anemia and apoptosis of erythroid precursors [61]. The short-term activation of the ISR can be shut-down by the dephosphorylation of eIF2 $\alpha$ by the GADD34 phosphatase [62]. When the cellular stress is too much for the cell to adapt, the ISR can lead to an induction of cell death. The ISR can induce apoptosis through transcriptional changes of members of the BCL-2 family. It has been 


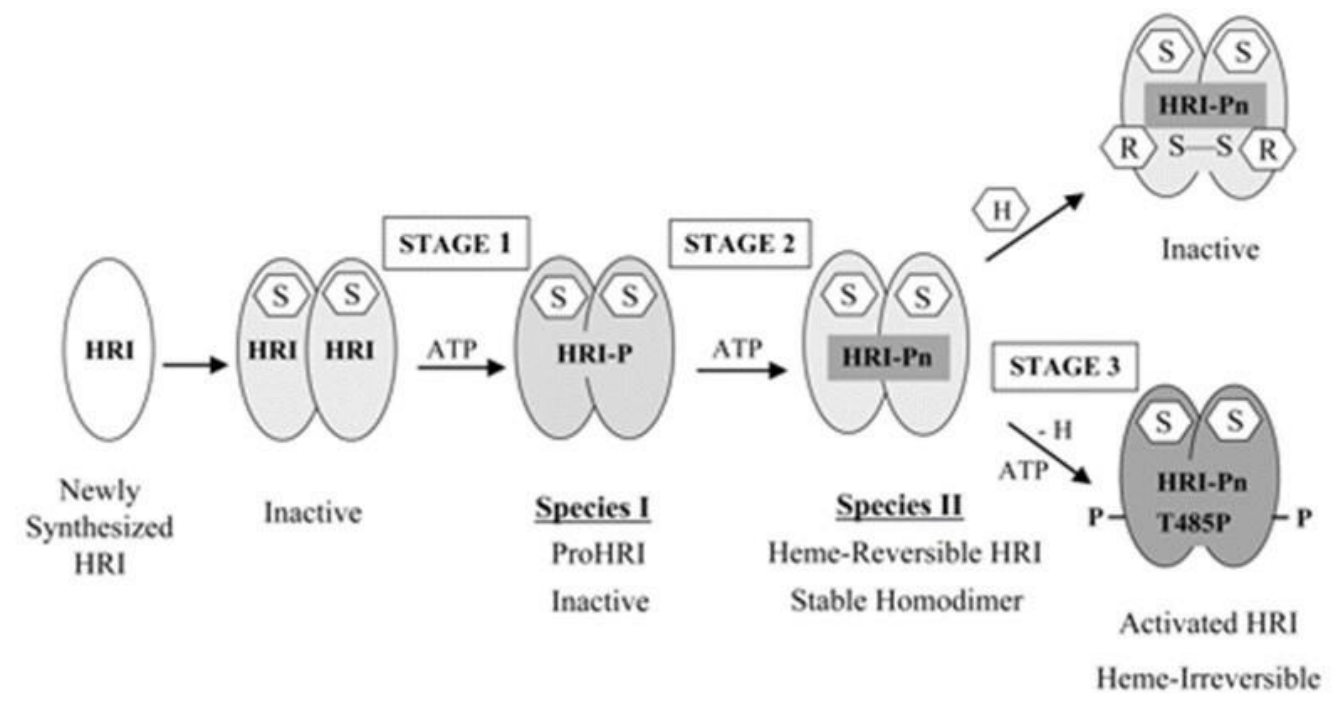

S Stable Heme; R Reversible Heme; P, Autophosporylation

Figure 1-3. A model of the activation of HRI by multiple autophosphorylation in three stages

Reprinted with permission from Rafie-Kolpin, M., A.P. Han, and J.J. Chen, Autophosphorylation of threonine 485 in the activation loop is essential for attaining eIF2alpha kinase activity of HRI. Biochemistry, 2003. 42(21): p. 6536-44. Copyright 2003 American Chemical Society. 
shown that CHOP induction leads to a transcriptional upregulation of Bcl2ll1 (encodes BIM) in response to ER stress [63]. NOXA and PUMA induction have also both been indicated as a consequence of ISR activation $[64,65]$. Although these may not be the only mechanisms through which the ISR can induce cell death, they highlight the possibility of exploiting the ISR pathway to target cancer cells.

\section{B-Lineage Acute Lymphoblastic Leukemia}

Acute lymphoblastic leukemia is a hematologic malignancy affecting both children and adults [66]. The estimated rate of new cases is 1.7 per 100,000 [67], and it is the most common form of childhood acute leukemia [66]. This disease arises from the proliferation and accumulation of $\mathrm{B}$ - or $\mathrm{T}$ - lineage lymphoid progenitors in the bone marrow [66]. B-ALL in children now has a cure rate of greater than $90 \%$; however, in those over 40 the cure rate is less than 50\% [68]. B-ALL can be classified into various subtypes based on aneuploidy, chromosomal rearrangements, or expression of fusion oncoproteins [68]. These can be further broken down based on prognosis, with Philadelphia chromosome positive $\left(\mathrm{Ph}^{+}\right)$and Philadelphia chromosome-like (Ph-like) both being classified as poor prognosis [68].

\section{Philadelphia Chromosome Positive B-ALL}

Philadelphia chromosome positive B-ALL results from a translocation between chromosomes $9 \mathrm{q} 34$ and 22q11 resulting in the fusion of the BCR-ABL oncoprotein (p185); this is a constitutively active tyrosine kinase $[69,70]$. The BCR-ABL protein leads to a variety of alterations in cellular pathways, including the Ras-mitogen-activated protein kinase, Janus-activated kinase-STAT, and phosphoinositide 3-kinase/AKT pathways, leading to increased proliferation and survival of the leukemic cells $[71,72]$. Although the prognosis of $\mathrm{Ph}^{+}$ALL patients has historically been poor, there have been improvements in recent years with the use of tyrosine kinase inhibitors (TKIs). Despite this improvement, many patients relapse and development of TKI resistance remains a challenge [71]. The prognosis of $\mathrm{Ph}^{+}$patients remains very poor after failure of TKIs, with a two-year overall survival of $20 \%$, highlighting the need for treatment improvements [71].

MCL-1 is important in many types of cancer and $\mathrm{Ph}^{+} \mathrm{B}-\mathrm{ALL}$ is no exception [5] . It is known that MCL-1 is required for the initiation and survival of $\mathrm{Ph}^{+} \mathrm{B}-\mathrm{ALL}$ cells [69]. After deletion of $M c l l$ the outgrowth of p185-transformed pre-B cells was delayed compared to that of $\mathrm{Mcll}$ wild-type cells, indicating the requirement of MCL-1 for in vitro leukemia initiation [69]. Further, Cre mediated deletion of Mcll resulted in the expansion of $\mathrm{p} 185^{+} \mathrm{B}-\mathrm{ALL}$ cells expressing $\mathrm{Mcll}^{\mathrm{wt}} \mathrm{cDNA}$, but not vector expressing cells indicating that MCL-1 is also required for the survival of these cells. This dependence on MCL-1 supports the idea that targeting MCL-1 in $\mathrm{Ph}^{+} \mathrm{B}-\mathrm{ALL}$ could be a reasonable treatment option. 


\section{Philadelphia Chromosome-Like B-ALL}

$\mathrm{Ph}$-like $\mathrm{B}-\mathrm{ALL}$ is characterized by a transcriptional profile similar to that of $\mathrm{Ph}^{+}$ B-ALL; however, these patients lack the expression of the BCR-ABL protein [68]. A variety of rearrangements, mutations, and copy number alterations are seen in this subtype that lead to activations of tyrosine kinases or cytokine receptor signaling. Most commonly, activations in JAK-STAT or ABL signaling pathways are seen; some examples of these mutations include rearrangements of CRLF2 or JAK2, or fusions in ABL class genes such as ABL1, ABL2, or PDGFRB [73]. Ph-like ALL is associated with a poor prognosis and is known to have a poorer 5-year event free survival when compared to non Ph-like ALL patients [74]. One challenge in treating these patients begins with the ability to accurately identify the kinase-activating alteration; this involves performing many assays (such as RT-PCR, FISH, or flow cytometric analyses) after identifying that the patient is classified as Ph-like [73]. Although treatment of these patients with TKIs may seem rational there is the potential challenge of development of resistance. Resistance to TKIs has already been a problem in treating $\mathrm{Ph}^{+}$ALL patients due to mutations such as the BCR-ABL T315I mutation [75]. Additionally, there has already been a PDGFRB mutation identified which confers resistance to ABL TKIs in $\mathrm{Ph}$-like ALL [76]. In addition to having a poor prognosis, this subtype of ALL also has a high prevalence, accounting for over $20 \%$ of adult ALL cases, further highlighting the need for improved treatment options of these patients [74]. 


\section{CHAPTER 2. METHODS ${ }^{1}$}

\section{Cells and Cell Culture}

Mouse p185 $4 r f$-null B-ALL (hereafter referred to as BCR-ABL ${ }^{+} \mathrm{B}-\mathrm{ALL}$ ) [69] and PAX5-JAK2, RCSD1-AB1, and RCSD1-ABL2 fusion-expressing Ph-like Ba/F3 cells [78] were grown in RPMI with 10\% fetal bovine serum, $55 \mu \mathrm{M}$ 2-mercaptoethanol, 2 $\mathrm{mM}$ glutamine, penicillin, and streptomycin (Invitrogen). Navitoclax and Venetoclax were obtained from Selleckchem. DHA was obtained from AvaChem Scientific. BTdCPU was obtained from Millipore and synthesized by the Department of Chemical Biology and Therapeutics (St. Jude Children's Research Hospital, SJCRH). The human $\mathrm{Ph}^{+}$leukemia cell lines OP-1, TOM-1, BV-173, and SUP-B15 were cultured in RPMI with $20 \%$ fetal bovine serum, $55 \mu \mathrm{M}$ 2-mercaptoethanol, $2 \mathrm{mM}$ glutamine, penicillin, and streptomycin. ISRIB (Sigma) was dissolved in DMSO for in vitro experiments.

\section{Genome-Wide CRISPR Screen}

Cas9-expressing $\mathrm{p} 185^{+} \mathrm{B}-\mathrm{ALL}$ cells were transduced with the Brie CRISPR KO library (Addgene \#73633) at a multiplicity of infection (MOI) of 0.5 with an sgRNA coverage of 400x [79]. One day after transduction, puromycin was added $(1 \mu \mathrm{g} / \mathrm{mL})$ to select for infected cells. Cells were then cultured in either DMSO or $10 \mu \mathrm{M}$ DHA containing media for 24 hours (h), followed by a $48 \mathrm{~h}$ culture in drug free media. As previously described, genomic DNA was extracted from the surviving cells (Qiagen DNeasy Blood and Tissue kit) and amplified by PCR for Illumina sequencing of sgRNAs by Nextseq [79]. MAGeCK was used to analyze the sequencing reads [80]. Enrichr was used for pathway analysis of the screen $[\underline{81}, \underline{82}]$.

\section{Validation CRISPR Screen}

A validation CRISPR KO library was generated by the Center for Advanced Genome Editing (SJCRH) containing 5 guides (different from those in the Brie library) for each of the top 35 hits in positive selection (FDR <1\%) and the top 13 hits in negative selection (FDR $<16 \%$ ) from the initial screen. Cas9-expressing BCR-ABL ${ }^{+}$B-ALL cells were transduced with this validation library at a multiplicity of infection of 0.5 with a sgRNA coverage of $>5000 x$. The drug treatment, sequencing and analysis were carried out in the same manner as the genome wide CRISPR screen.

\footnotetext{
${ }^{1}$ Modified from final submission with permission. Smith, K.H., et al., The Heme-Regulated Inhibitor Pathway Modulates Susceptibility of Poor Prognosis B-Lineage Acute Leukemia to BH3-Mimetics. Mol Cancer Res, 2020. https://doi.org/10.1158/1541-7786.MCR-20-0586. [77]
} 


\section{Knockout Cell Line Creation}

BCR-ABL ${ }^{+} \mathrm{B}-\mathrm{ALL}$ knockout cell lines were generated using CRISPR-Cas9 technology. Briefly, $1 \times 10^{6}$ mouse $\mathrm{BCR}-\mathrm{ABL}^{+} \mathrm{B}-\mathrm{ALL}$ cells were transiently cotransfected with precomplexed ribonuclear proteins (RNPs) consisting of $100 \mathrm{pmol}$ of chemically modified sgRNA (Synthego) and 35 pmol of Cas9 protein (SJCRH Protein Production Core). Additionally, $200 \mathrm{ng}$ of pMaxGFP was co-transfected via nucleofection (Lonza, 4D-Nucleofector ${ }^{\mathrm{TM}} \mathrm{X}$-unit) using solution $\mathrm{P} 3$ and program CM137 in small cuvettes according to the manufacturer's recommended protocol. Five days post nucleofection, cells were single-cell sorted by FACs to enrich for $\mathrm{GFP}^{+}$(transfected) cells, clonally selected, and verified for the desired out-of-frame indel modifications via targeted deep sequencing on a Miseq Illumina sequencer. NGS analysis of clones was performed using CRIS.py [83]. Two knockout clones for each gene were identified. BV173 genetically modified cell pools were created by transiently co-transfecting 400,000 cells with precomplexed ribonuclear proteins (RNPs) consisting of $100 \mathrm{pmol}$ of chemically modified sgRNA (Synthego) and 35 pmol of Cas9 protein (St. Jude Protein Production Core) via nucleofection (Lonza, 4D-Nucleofector ${ }^{\mathrm{TM}} \mathrm{X}$-unit) using solution P3 and program CA-137 in small cuvettes according to the manufacturers recommended protocol. Five days post nucleofection, a portion of cells were harvested and sequenced via targeted NGS and analyzed using CRIS.py as described above. NGS analysis indicated 89\% total indels and 80\% out-of-frame indels for the BV-173 edited cell pool. Sequences of sgRNA can be found in Table 2-1. MCL-1 overexpressing cells were previously generated by retroviral transduction [32, 69].

\section{Immunoblotting and Antibodies}

Protein expression was assessed as previously described [84]. Antibodies used were: anti-MCL-1 (Rockland Immunochemical), anti-human MCL-1, anti-PERK, antiATF6, anti-IRE1, anti-CHOP, anti-ATF4, anti-BCL-XL, anti-Phospho-eIF2 $\alpha$, anti-eIF2 $\alpha$, anti-PUMA, anti-p53 (Cell Signaling), anti-HRI, anti- $\gamma \mathrm{H} 2 \mathrm{AX}$ (Abcam), anti-NOXA (Sigma), anti-BIM, anti-BCL-2 (BD Pharmingen), and anti-Actin (Millipore). Antirabbit or anti-mouse horseradish peroxidase-conjugated secondary antibodies were from Jackson Immunochemical. Anti-hamster horseradish peroxidase-conjugated secondary antibody was from Invitrogen. Immunoblots were developed using Odyssey Fc imaging system from LI-COR Biosciences. All experiments are representative of $\geq 3$ independently performed assays.

\section{Measuring Interaction Between HRI and Heme}

Absorption spectra were acquired using a Nanodrop 1000 spectrophotometer (Thermo Fisher) at room temperature. All dilutions were made in Enzyme Dilution Buffer (EDB) consisting of $50 \mathrm{mM}$ Tris (pH 7.4), $150 \mathrm{mM} \mathrm{NaCl}, 0.1 \mathrm{mM}$ EDTA, 25\% glycerol. HRI $(0.05 \mu \mathrm{g} / \mathrm{ul})$ was obtained from ABM (Z500115). Heme (Frontier Scientific) and DHA were dissolved in DMSO to make an initial $10 \mathrm{mM}$ stock, then 
Table 2-1. $\quad$ sgRNA sequences for knockout cell lines

\begin{tabular}{ll}
\hline \multicolumn{1}{c}{ Name } & \multicolumn{1}{c}{ Sequence (5' to 3') } \\
\hline $\begin{array}{l}\text { mErn1 KO BCR-ABL } \\
\text { mErn1 sgRNA spacer }\end{array}$ & AGL reagents \\
mErn1.NGS.F partial Illumina adaptors (upper case) & CACTCTTTCCCTACACGACGCTCTTCCGATCTagtggattgacatgaaggcgt \\
mErn1.NGS.R partial Illumina adaptors (upper case) & GTGACTGGAGTTCAGACGTGTGCTCTTCCGATCTggagtgcaagtccgtttcctc \\
mAtf6 KO BCR-ABL ${ }^{+}$B-ALL reagents & \\
mAtf6 sgRNA spacer & UUCCUUCAGUGGCUCUACAG \\
mAtf6.NGS.F partial Illumina adaptors (upper case) & CACTCTTTCCCTACACGACGCTCTTCCGATCTtgtttcactgcaggaggagt \\
mAtf6.NGS.R partial Illumina adaptors (upper case) & GTGACTGGAGTTCAGACGTGTGCTCTTCCGATCTaagcttaatccaagatttgccttt \\
mEif2ak3 KO BCR-ABL ${ }^{+}$B-ALL reagents & \\
mEif2ak3 sgRNA spacer & \\
mEif2ak3.NGS.F partial Illumina adaptors (upper case) & CAGUUUGCUUGCUCCCGCGU \\
mEif2ak3.NGS.R partial Illumina adaptors (upper case) & GTGACTGGAGTTCAGACGTGTGCTCTTCCGATCTcgcagttccacgtcgtcatc \\
& \\
mEifak1 KO BCR-ABL ${ }^{+}$B-ALL reagents & \\
mEifak1 sgRNA spacer & UGUUUGGAGUGGAGCGGGUU \\
mEifak1.NGS.F partial Illumina adaptors (upper case) & CACTCTTTCCCTACACGACGCTCTTCCGATCTaccagactcaccagaccgctctgcc \\
mEifak1.NGS.R partial Illumina adaptors (upper case) & GTGACTGGAGTTCAGACGTGTGCTCTTCCGATCTacacaccagctgcttctcagagtca \\
& \\
hEIF2AK1 KO BV-173 reagents & \\
hEIF2AK1 sgRNA spacer & UUGUUGGCUAUCACACCGCG \\
hEIF2AK1.NGS.F partial Illumina adaptors (upper case) & CACTCTTTCCCTACACGACGCTCTTCCGATCTaggggccatctgtattttgctctgga \\
hEIF2AK1.NGS.R partial Illumina adaptors (upper case) & GTGACTGGAGTTCAGACGTGTGCTCTTCCGATCTaatggtgtgaacccaggaggcggag \\
\hline
\end{tabular}


further diluted in EDB to make 10X final stock immediately prior to addition. Enzyme was brought to $5 \mu \mathrm{M}$ with heme and to the indicated concentration of DHA then incubated at room temperature for 30 minutes. Controls, without heme or DHA, had an equivalent volume of EDB added. All measurements were made after blanking with buffer (control) or with buffer containing the equivalent concentrations of heme and DHA as the measured sample. Absorbance readings between 220 and $750 \mathrm{~nm}$ were made for each sample, and Soret peak height for each independent experiment was determined by recording peak absorbance $(420 \mathrm{~nm})$ from 3 or more technical replicates per value.

\section{Heme Measurements}

Cellular heme measurements were made by reverse phase HPLC after pellets were extracted with acetone, acidified by the addition of $20 \% 1.6 \mathrm{~N} \mathrm{HCl}$, together with 10 pmol mesoporphyrin per sample added as an internal standard. Extracts were centrifuged at 21,100 $\times \mathrm{g}$ for $10 \mathrm{~min}$. after which pellets were discarded. From the supernatants, heme was separated from other porphyrins on a Shimadzu system (CBM20A system controller, Shimadzu), using a mobile phase of acetonitrile in water containing $0.05 \%$ trichloroacetic acid at $1 \mathrm{ml} / \mathrm{min}$ on a reverse-phase $\mathrm{C} 18$ column (Sigma), applying a 30-66\% linear acetonitrile gradient over 5 min followed by a 66$90 \%$ linear gradient over 20 min. Heme was determined by measuring absorbance at 400 $\mathrm{nm}$ (Shimadzu, SPD-20AV). The concentration and identity of heme (tR=7.4 min.) was made by comparison with hemin (Frontier Scientific, H651-9) standards extracted analogously to samples. Calculations were made by normalizing the peak area for heme with internal standard peak values for samples then calculating from a linear curve made from hemin standards similarly normalized to the internal standard. Limit of detection was approximately 1 pmol with a linear range or detection to at least $1 \mathrm{nmol}$.

\section{Cell Death Experiments}

Cells were seeded in 96-well plates and drugs (DHA, BTdCPU, ABT-199, ABT263 solubilized in DMSO or DMSO vehicle controls) were added at the indicated concentrations. Aminolevulinic acid (ALA), a precursor of heme synthesis, or succinylacetone (SA), an inhibitor of heme synthesis, (both from Sigma) were solubilized in water and added at indicated concentrations to alter cellular heme levels [85]. Cell viability was determined by staining with Annexin-V-APC and propidium iodide (BD Biosciences) and measured by flow cytometry as previously described [32].

\section{Response Surface Modeling}

Response surface modeling, implemented in Matlab version R2016a (Mathworks), was used to determine changes in the response of two drugs given in combination [86-88]. A drug combination was considered either synergistic or antagonistic if the interaction term $(\alpha)$ describing the change in response relative to the 
additive model was either positive or negative, respectively. Two interaction terms $(\alpha)$ were considered different if their difference was statistically different from zero based on a two-tailed z-test.

\section{Patient-Derived Xenograft (PDX) Leukemia}

Leukemia from adult patients with BCR-ABL1 ${ }^{+}$and EBF1-PDGFRB Ph-like ALL obtained from the Eastern Cooperative Oncology Group E2993 study (ClinicalTrials.gov identifier NCT00002514) and from the University Health Network (Toronto, CA) were transplanted into un-irradiated immunodeficient NOD.CgPrkdc $^{\text {scid }}$ Il2 $2 \mathrm{rg}^{\mathrm{tm} 1 \mathrm{Wj}} / \mathrm{SzJ}$ (NSG) mice (Jackson Laboratories) for 8-10 weeks prior to reisolation [89-91]. Mice were bred and utilized in accordance with SJCRH animal care and use committee (SJCRHACUC).

\section{Treatment of Leukemia in Recipient Mice}

BCR-ABL ${ }^{+} \mathrm{B}-\mathrm{ALL}$ were injected $\left(2 \times 10^{5}\right)$ into non-conditioned, 6-8-week-old, female C57BL/6 recipients (Jackson Laboratory). Five days after the transfer, recipients were treated with BTdCPU via intraperitoneal route and ABT-263 by oral gavage. Navitoclax was formulated in a mixture of 60\% Phosal 50 PG, 30\% PEG 400, and 10\% $\mathrm{EtOH}$ and dosed at $100 \mathrm{mg} / \mathrm{kg} / \mathrm{day}$ as previously described [92]. $400 \mathrm{mg} / \mathrm{kg} / \mathrm{day}$ of BTdCPU was administered in $30 \mu \mathrm{L}$ DMSO. Treatment was given daily for 14 days (days 5-18 after leukemia injection) during and after which the mice were monitored. Mice were bred and utilized in accordance with SJCRHACUC.

\section{Pathology and Immunohistochemistry}

All tissues were fixed in formalin, embedded in paraffin, sectioned at $4 \mu \mathrm{m}$, mounted on positively charged glass slides (Superfrost Plus; Thermo Fisher Scientific), and dried at $60^{\circ} \mathrm{C}$ for $20 \mathrm{~min}$ before dewaxing and staining with hematoxylin and eosin (H\&E) using standard methods. For immunohistochemical staining, the primary antibodies used in this study included anti-B220 (BD Biosciences) and anti-PAX5 (Abcam). Tissue sections underwent antigen retrieval in a prediluted Cell Conditioning Solution (CC1) (Ventana Medical Systems) for $32 \mathrm{~min}$, and the OmniMap anti-Rabbit HRP kit (Ventana Medical Systems) and ChromoMap DAB (Ventana Medical Systems) were used for detection. All sections were examined by a pathologist blinded to the experimental group assignments. 


\section{Measurement of Reactive Oxygen Species}

BCR-ABL ${ }^{+}$cells were treated with ROS scavengers (MnTMPYP, NAC, MnTBAP) for $2 \mathrm{~h}$ prior to DHA treatment. Cells were stained with $5 \mu \mathrm{M}$ dihydroethidium (DHE, Sigma) for 30 minutes and analyzed by flow cytometry. 


\section{CHAPTER 3. RESULTS ${ }^{2}$}

\section{CRISPR Screen Identifies Pathways Required for DHA-Induced Apoptosis}

Previous efforts to mechanistically address how DHA triggered the repression of MCL-1 revealed a gene expression signature consistent with the induction of the endoplasmic reticulum (ER) stress pathway [32]. To further interrogate this pathway, the three canonical branches of the cellular ER stress pathway were genetically ablated in mouse $185^{+}$Arf-null B-ALL (hereafter referred to as BCR-ABL ${ }^{+}$B-ALL) using CRISPR/Cas9 targeting (Figure 3-1). Despite loss of the genes encoding the IRE1, ATF6, or PERK branches of the ER stress pathway, DHA treatment still triggered MCL1 repression in mouse $\mathrm{BCR}-\mathrm{ABL}^{+} \mathrm{B}-\mathrm{ALL}$, indicating that none of the canonical ER stress pathway signaling arms is singularly responsible for repressing MCL-1 expression in response to DHA treatment (Figure 3-1).

To elucidate which cellular signaling pathways are activated by DHA to induce cell death in mouse BCR-ABL ${ }^{+}$B-ALL cells, an unbiased genetic screen was conducted in which Cas9-expressing mouse BCR-ABL ${ }^{+} \mathrm{B}$-ALL cells were stably transduced with the Brie knockout library (78,637 sgRNAs targeting 19,674 genes) [79]. The cells were treated with $10 \mu \mathrm{M}$ DHA for 24 hours (h) and cultured in drug free media for an additional $48 \mathrm{~h}$ to allow outgrowth of resistant cells (Figure 3-2A). After DHA treatment, BCR-ABL ${ }^{+}$B-ALL cell viability was approximately $40 \%$ in contrast to vehicle control treated cells that maintained $>95 \%$ cell viability (Figure 3-2B). After treatment, genomic DNA was isolated from the viable BCR-ABL ${ }^{+} \mathrm{B}-\mathrm{ALL}$ cells, amplified and subjected to Illumina sequencing to determine sgRNAs enrichment in the surviving cells. MAGeCK analysis of sequencing data identified 35 genes whose targeting sgRNAs were enriched in DHA-resistant cells at a false discovery rate of $<1 \%$ [80], indicating that loss of expression of these genes generated resistance to $10 \mu \mathrm{M}$ DHA (Figure 3-2C and Table 3-1). Enrichr gene set enrichment analysis was performed [81, 82] using these 35 genes, to identify genetic pathways implicated in DHA resistance. Multiple pathways related to heme synthesis/metabolism and apoptosis were identified (Figure 3-2D and Table 3-1); in fact, most genes involved in the heme synthesis pathway were among the top hits [93] (Figure 3-3). These data implicate that either the heme synthesis or intrinsic apoptotic pathway is required for induction of apoptosis in $\mathrm{BCR}-\mathrm{ABL}^{+} \mathrm{B}-\mathrm{ALL}$ cells in response to $10 \mu \mathrm{M}$ DHA.

To validate the hits identified from the primary genome-wide screen a secondary targeted screen was performed using a validation library composed of five, new sgRNA guides per gene for the top 35 hits in positive selection (FDR <1\%) and the top 13 hits in negative selection (FDR $<16 \%$ ). The validation screen was carried out in the same way as the original genome-wide screen. After MAGeCK analysis, 8 of the 10 genes involved

\footnotetext{
${ }^{2}$ Modified from final submission with permission. Smith, K.H., et al., The Heme-Regulated Inhibitor Pathway Modulates Susceptibility of Poor Prognosis B-Lineage Acute Leukemia to BH3-Mimetics. Mol Cancer Res, 2020. https://doi.org/10.1158/1541-7786.MCR-20-0586. [77]
} 

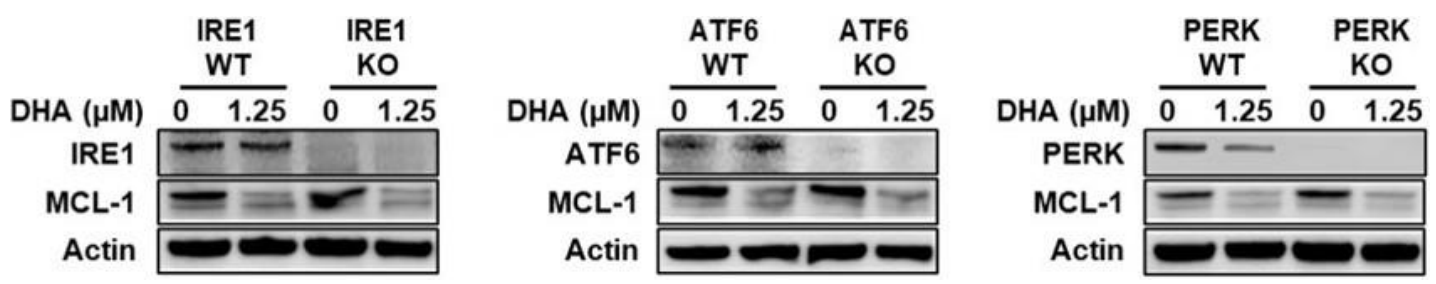

Figure 3-1. Stress pathways in cellular response to DHA

Wild-type (WT) or BCR-ABL ${ }^{+}$B-ALL cells deficient (KO) for ERNI (encodes IRE1), ATF6, or Eif2ak3 (encodes PERK) were treated with $1.25 \mu \mathrm{M}$ DHA for 9h and MCL-1 expression was determined by immunoblotting with indicated antibodies. 
A.

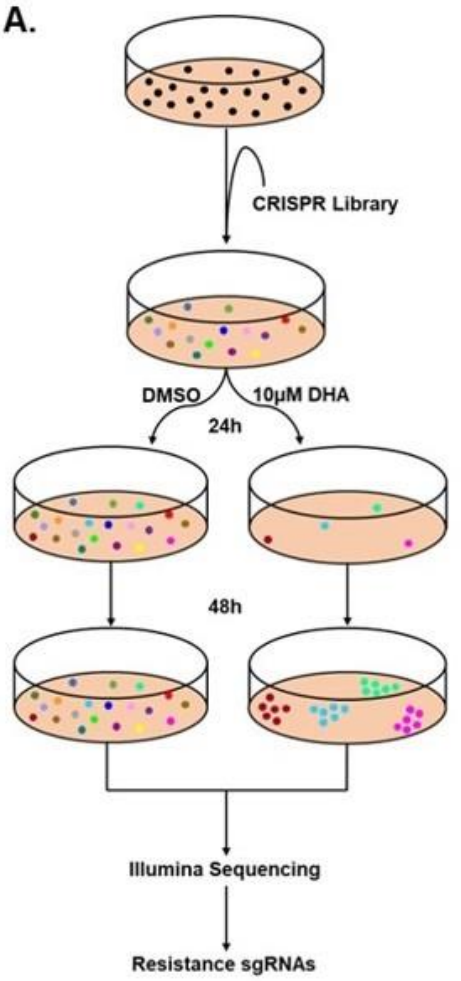

D.

Heme biosynthesis

Metabolism of porphyrins

Cytochrome c-mediated apoptotic response

Activation of caspases through apoptosome-mediated cleavage

Apoptotic factor-mediated response

Metabolism

Apoptosis

Programmed Cell Death

Intrinsic Pathway for Apoptosis

Activation of DNA fragmentation factor
B.

DHA Treatment

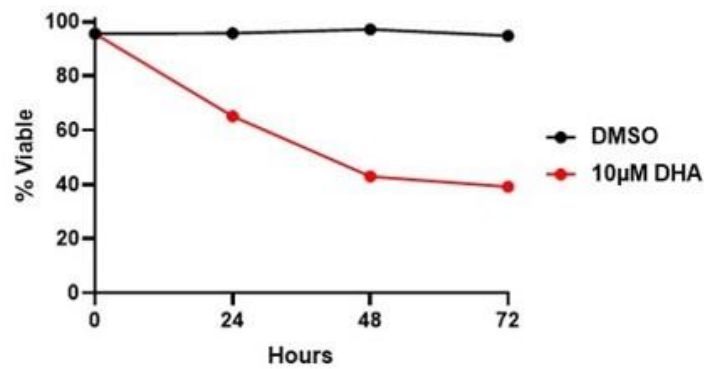

C.

Enriched Genes

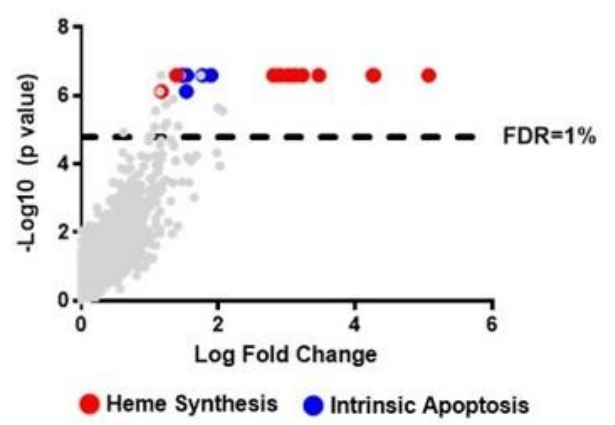

E. Log Fold Change

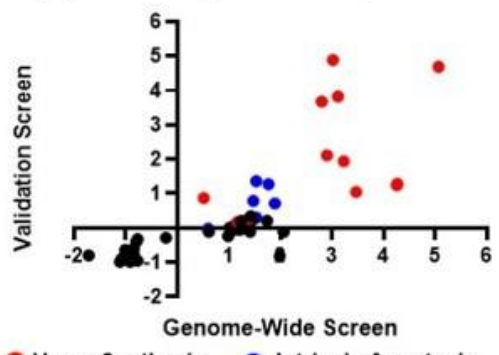

Heme Synthesis
P-value $<1.5 \mathrm{E} 06$

Heme Synthesis/Metabolism

Intrinsic Apoptosis

Figure 3-2. CRISPR screen identifies the requirement of the heme synthesis pathway for DHA-induced apoptosis

(A) BCR-ABL ${ }^{+} \mathrm{B}-\mathrm{ALL}$ cells containing the Brie CRISPR knockout library were treated with DHA or DMSO for $24 \mathrm{~h}$ and then cultured for an additional $48 \mathrm{~h}$ in drug free media. Cells that survived the drug treatment were harvested and sequenced to determine genes involved in resistance. (B) Percentage of viable cells treated with either $10 \mu \mathrm{M}$ DHA or DMSO was measured by trypan blue staining. Cells were under treatment conditions for $24 \mathrm{~h}$ and then cultured in drug free media for an additional 48h. (C) MAGeCK was used to determine sgRNAs enriched for in the DHA treated sample. (D) Enrichr was used to determine pathways involved in resistance to DHA. (E) BCR-ABL ${ }^{+}$B-ALL cells containing the validation screen CRISPR library were treated with DHA for $24 \mathrm{~h}$ and cultured for an additional $48 \mathrm{~h}$ in drug free media. Surviving cells were harvested and the DNA sequenced. MAGeCK was used to determine sgRNAs enriched for in the DHA treated sample. The enriched genes from both the validation screen and original genomewide screen were then compared. 
Table 3-1. Top 35 hits from genome wide CRISPR screen, determined by MAGeCK

\begin{tabular}{|c|c|c|c|c|c|c|}
\hline Gene id & pos|score & pos|p-value & pos|fdr & pos|rank & pos|lfc & $\begin{array}{c}\text { Related } \\
\text { pathway }\end{array}$ \\
\hline Ppox & $3.25 \mathrm{E}-16$ & $2.52 \mathrm{E}-07$ & 0.000248 & 1 & 4.2704 & Heme \\
\hline Alad & $1.18 \mathrm{E}-15$ & $2.52 \mathrm{E}-07$ & 0.000248 & 2 & 5.071 & Heme \\
\hline$P d x k$ & $1.53 \mathrm{E}-14$ & $2.52 \mathrm{E}-07$ & 0.000248 & 3 & 4.2593 & Heme \\
\hline $\mathrm{Hmbs}$ & $4.25 \mathrm{E}-14$ & $2.52 \mathrm{E}-07$ & 0.000248 & 4 & 3.0221 & Heme \\
\hline Срох & $9.66 \mathrm{E}-13$ & $2.52 \mathrm{E}-07$ & 0.000248 & 5 & 3.1179 & Heme \\
\hline Apaf1 & $1.02 \mathrm{E}-12$ & $2.52 \mathrm{E}-07$ & 0.000248 & 6 & 1.7716 & Apoptosis \\
\hline Casp9 & $1.43 \mathrm{E}-12$ & $2.52 \mathrm{E}-07$ & 0.000248 & 7 & 1.8948 & Apoptosis \\
\hline $\operatorname{Trp53}$ & $1.64 \mathrm{E}-12$ & $2.52 \mathrm{E}-07$ & 0.000248 & 8 & 1.4796 & Apoptosis \\
\hline Casp3 & $4.93 \mathrm{E}-12$ & $2.52 \mathrm{E}-07$ & 0.000248 & 9 & 1.4571 & Apoptosis \\
\hline$D f f b$ & $9.85 \mathrm{E}-12$ & $2.52 \mathrm{E}-07$ & 0.000248 & 10 & 1.5408 & Apoptosis \\
\hline Glrx 5 & $1.65 \mathrm{E}-11$ & $2.52 \mathrm{E}-07$ & 0.000248 & 11 & 3.4678 & \\
\hline Dffa & $1.91 \mathrm{E}-11$ & $2.52 \mathrm{E}-07$ & 0.000248 & 12 & 1.4907 & Apoptosis \\
\hline Ube $2 m$ & $3.83 \mathrm{E}-11$ & $2.52 \mathrm{E}-07$ & 0.000248 & 13 & 1.4299 & \\
\hline Alas1 & $2.62 \mathrm{E}-10$ & $2.52 \mathrm{E}-07$ & 0.000248 & 14 & 2.9046 & Heme \\
\hline Fech & 2.93E-09 & $2.52 \mathrm{E}-07$ & 0.000248 & 15 & 2.8039 & Heme \\
\hline Cluh & $5.67 \mathrm{E}-09$ & $2.52 \mathrm{E}-07$ & 0.000248 & 16 & 1.1662 & \\
\hline Peol & $9.63 \mathrm{E}-09$ & $2.52 \mathrm{E}-07$ & 0.000248 & 17 & 1.4576 & \\
\hline Uros & $1.92 \mathrm{E}-08$ & $2.52 \mathrm{E}-07$ & 0.000248 & 18 & 3.228 & Heme \\
\hline Pnpo & 4.29E-08 & $2.52 \mathrm{E}-07$ & 0.000248 & 19 & 1.3911 & Heme \\
\hline Eefsec & $5.42 \mathrm{E}-08$ & $2.52 \mathrm{E}-07$ & 0.000248 & 20 & 1.7452 & \\
\hline Cycs & $1.49 \mathrm{E}-07$ & $7.55 \mathrm{E}-07$ & 0.000646 & 21 & 1.5336 & Apoptosis \\
\hline Suclgl & $1.50 \mathrm{E}-07$ & $7.55 \mathrm{E}-07$ & 0.000646 & 22 & 1.1732 & Heme \\
\hline Fam122a & $1.53 \mathrm{E}-07$ & $7.55 \mathrm{E}-07$ & 0.000646 & 23 & 1.149 & \\
\hline Elp5 & $3.06 \mathrm{E}-07$ & $1.26 \mathrm{E}-06$ & 0.001031 & 24 & 1.3464 & \\
\hline Xpo7 & $4.38 \mathrm{E}-07$ & $1.76 \mathrm{E}-06$ & 0.001386 & 25 & 1.2388 & \\
\hline Pcytla & $5.41 \mathrm{E}-07$ & $2.26 \mathrm{E}-06$ & 0.001714 & 26 & 1.9908 & \\
\hline Pstk & $8.82 \mathrm{E}-07$ & $2.77 \mathrm{E}-06$ & 0.001945 & 27 & 1.084 & \\
\hline Wbscrl6 & $8.96 \mathrm{E}-07$ & $2.77 \mathrm{E}-06$ & 0.001945 & 28 & 2.0637 & \\
\hline Trub2 & $1.97 \mathrm{E}-06$ & $6.29 \mathrm{E}-06$ & 0.004268 & 29 & 1.247 & \\
\hline Ttc14 & $2.29 \mathrm{E}-06$ & $7.80 \mathrm{E}-06$ & 0.005105 & 30 & 1.415 & \\
\hline Hnrnpa2bl & $2.51 \mathrm{E}-06$ & 8.30E-06 & 0.005105 & 31 & 1.2129 & \\
\hline Mthfd2 & $2.57 \mathrm{E}-06$ & 8.30E-06 & 0.005105 & 32 & 1.0731 & \\
\hline Lztrl & $3.17 \mathrm{E}-06$ & $1.18 \mathrm{E}-05$ & 0.007051 & 33 & 0.61398 & \\
\hline Slc25a32 & $4.13 \mathrm{E}-06$ & $1.48 \mathrm{E}-05$ & 0.008591 & 34 & 1.017 & \\
\hline Ubpl & $4.45 \mathrm{E}-06$ & $1.64 \mathrm{E}-05$ & 0.009194 & 35 & 0.98916 & \\
\hline
\end{tabular}

Pos|Score: The RRA value of the gene in positive selection; Pos | p-value: raw p-value of this gene in positive selection; Pos | fdr: false discovery rate of this gene in positive selection; Pos | rank: ranking of each gene in positive selection; Pos | lfc: log fold change of the gene in positive selection; Related Pathway: category of gene as determined by Enrichr/GSEA. 


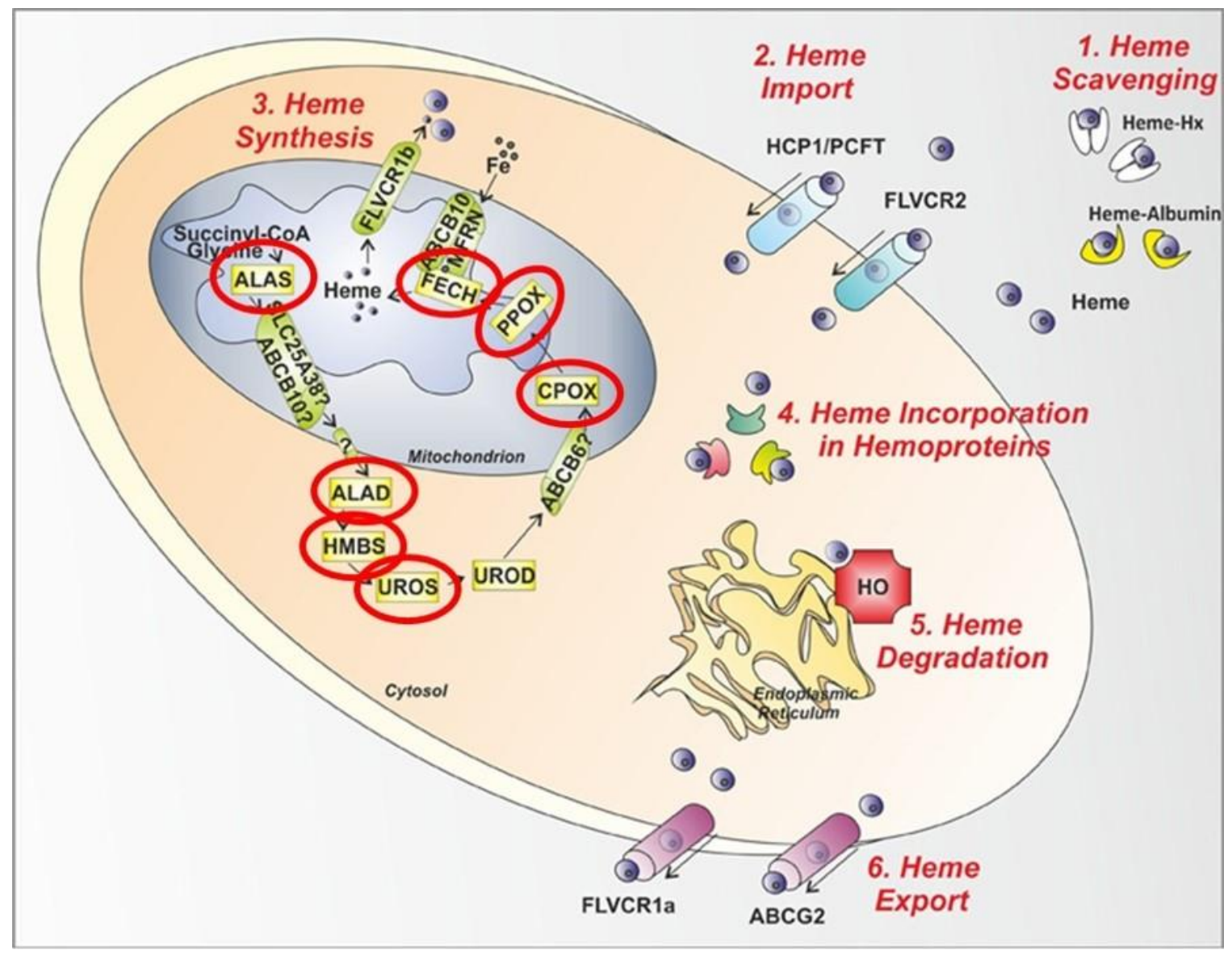

\section{Figure 3-3. Control steps in heme metabolism}

The main mechanisms involved in the control of heme levels outside, inside and across the cell are illustrated. Genes circled in red indicate that they were hits in the genomewide CRISPR screen. Modified with permission from Chiabrando, Vinchi, Fiorito, Mercurio and Tolosano. Chiabrando, D., et al., Heme in pathophysiology: a matter of scavenging, metabolism and trafficking across cell membranes. Front Pharmacol, 2014. 5: p. 61. 
in heme synthesis and 4 of the 7 apoptosis-related genes identified in the genome-wide screen were found to be top hits in the validation screen $(\mathrm{FDR}<12 \%)$ (Figure 3-2E). These data strongly suggest that DHA requires the heme synthesis pathway to kill BCR$\mathrm{ABL}^{+} \mathrm{B}-\mathrm{ALL}$ cells.

Since sgRNAs targeting the heme synthesis pathway were associated with resistance of $\mathrm{BCR}-\mathrm{ABL}{ }^{+} \mathrm{B}-\mathrm{ALL}$ cells to DHA treatment, we hypothesized that repressing heme levels pharmacologically should also render BCR-ABL ${ }^{+} \mathrm{B}-\mathrm{ALL}$ cells resistant to DHA treatment. To test this hypothesis, we cultured wild-type or MCL-1 overexpressing (OE) BCR-ABL ${ }^{+}$B-ALL cells in succinylacetone (SA), an inhibitor of heme synthesis [85]. This rendered the wild-type cells more resistant to death induced by culture with DHA (Figure 3-4A through C). Conversely, wild-type or MCL-1 OE BCR-ABL ${ }^{+} \mathrm{B}-$ ALL cells were cultured in aminolevulinic acid (ALA), a precursor of heme synthesis [85], to increase cellular heme levels. The ALA treatment made the wild-type, but not MCL-1 OE, cells more sensitive to DHA induced apoptosis (Figure 3-4A through C). DHA, alone or in combination with ALA or SA, had little effect on MCL-1 OE cell viability, indicating that the changes in toxicity seen in wild-type cells when DHA was combined with ALA or SA are due to changes in endogenous MCL-1 expression. Since DHA acts to repress MCL-1 translation in leukemic cells [32], we tested whether pharmacological manipulation of cellular heme levels could affect MCL-1 repression in response to DHA treatment. The combination of ALA and DHA caused MCL-1 repression like that observed with DHA alone (Figure 3-4D). In contrast, the inhibition of heme synthesis by SA substantially blunted MCL-1 repression triggered by DHA (Figure 3-4D). Taken together, these data indicate that cellular heme is needed both for DHA to induce apoptosis and to repress MCL-1 expression.

\section{DHA Induces an HRI-Mediated eIF2 $\alpha$ Phosphorylation and MCL-1 Repression}

The observation that heme is required for DHA induced apoptosis and repression of MCL-1 prompted us to further examine how the heme pathway could trigger the translational repression of MCL-1. The phosphorylation of eIF2 $\alpha$ inhibits cap-dependent translation by triggering the integrated stress response [94]. Indeed, when BCR-ABL ${ }^{+} \mathrm{B}-$ ALL cells were treated with DHA, eIF $2 \alpha$ was phosphorylated and the protein expression of both ATF4 and the CHOP transcription factor were induced (Figure 3-5A).

Concomitantly, MCL-1 expression was repressed, but the expression of other antiapoptotic BCL-2 family members, such as BCL-XL or BCL-2, were unaffected (Figure 3-5A). There was an increase in the pro-apoptotic BCL-2 family members NOXA and PUMA in response to DHA; however, BIM expression was unchanged (Figure 3-5A). Additionally, DHA, alone and in combination with ALA, induces the integrated stress response, as shown by the phosphorylation of eIF $2 \alpha$ and induction of its downstream targets, ATF4 and CHOP (Figure 3-4D). Decreasing cellular heme levels with SA treatment blunted the induction of the integrated stress response by DHA (Figure 3-4D). There are four known eIF2 $\alpha$ kinases, GCN2, PKR, PERK, and the heme regulated inhibitor (HRI) [52]. PERK-deficient cells still repressed MCL-1 in response to DHA 
A.

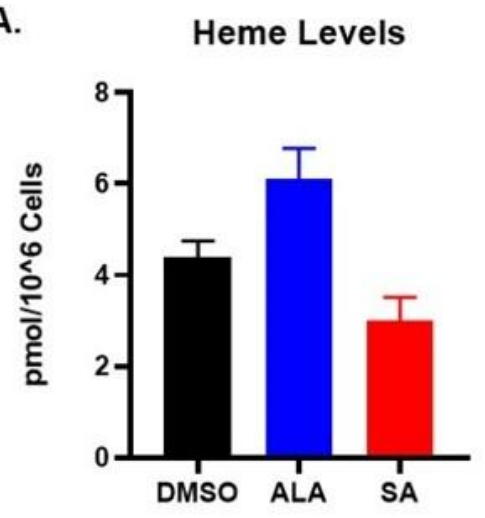

D.

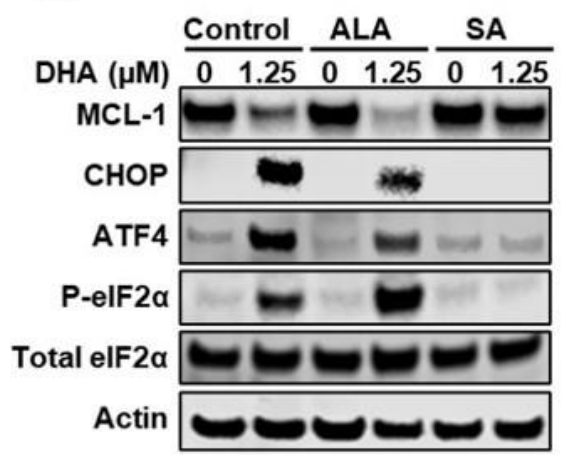

B.

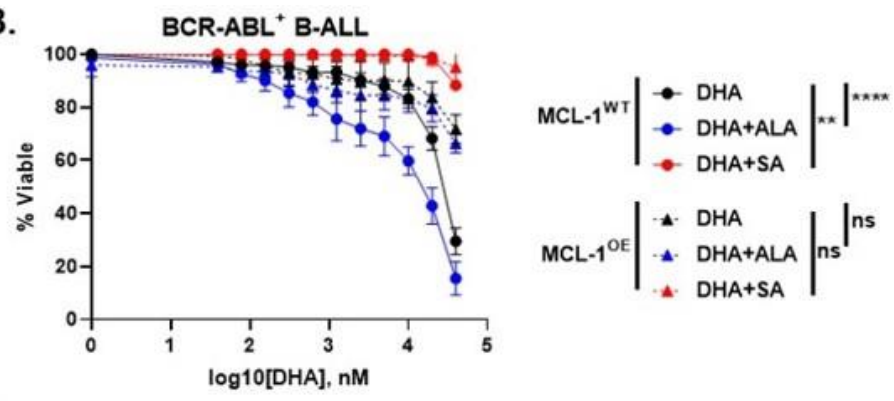

C. Actin

Figure 3-4. Effects of heme synthesis alterations on cellular response to DHA (A) Heme levels were measured in BCR-ABL ${ }^{+} \mathrm{B}-\mathrm{ALL}$ cells treated with DMSO, $62.5 \mu \mathrm{M}$ (Aminolevulinic acid) ALA, or $62.5 \mu \mathrm{M}$ (succinylacetone) SA for 24h. (B) MCL-1 wildtype (WT) or overexpressing (OE) cells were treated with a combination of DHA and $62.5 \mu \mathrm{M}$ of Aminolevulinic acid (ALA) or succinylacetone (SA) for 24h. Viability was measured using Annexin-V and propidium iodide staining. Data are the average of three experiments and error bars are SEM. Two-way ANOVA with Bonferroni multiple comparisons indicates significance $\mathrm{P}<0.0001 * * * *, \mathrm{P}<0.01 * *$ between the DHA alone and DHA+ALA or DHA+SA treatments at $10 \mu \mathrm{M}$ DHA. (C) MCL-1 expression in WT or OE cells was determined by immunoblotting with indicated antibodies. (D) Cells were treated with DHA alone or a combination of DHA and $62.5 \mu \mathrm{M}$ of Aminolevulinic acid (ALA) or succinylacetone (SA) for $24 \mathrm{~h}$. Protein expression was determined by immunoblotting with indicated antibodies. 


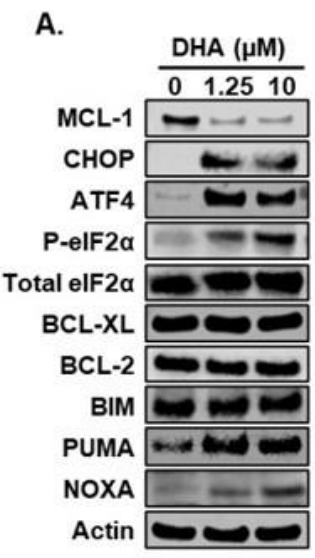

E.
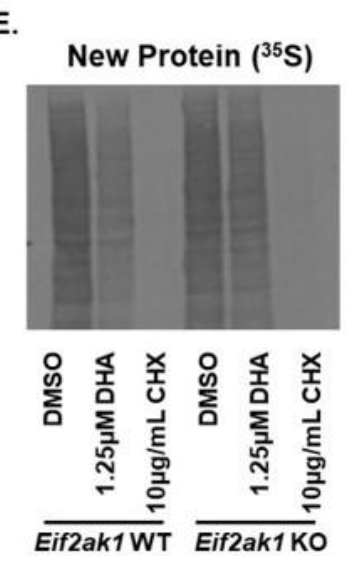

B.

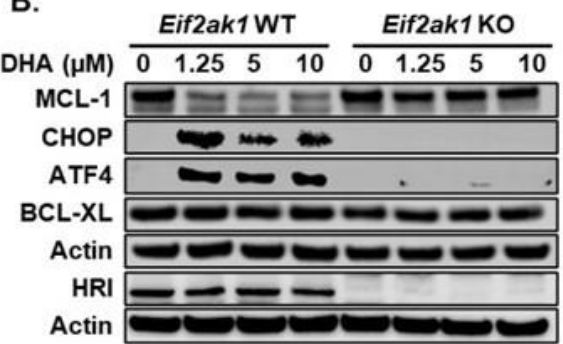

C.

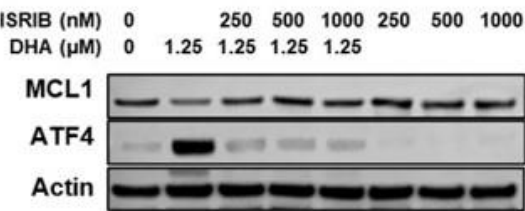

D.

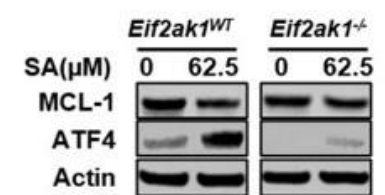

F.

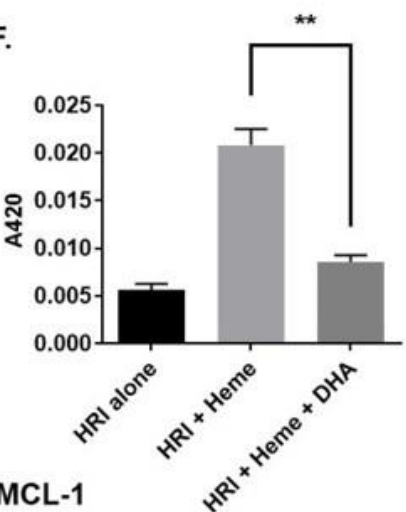

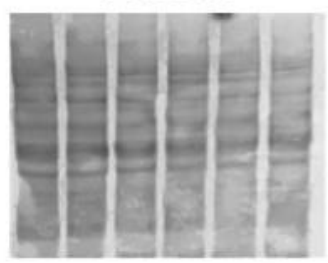

Western Blot MCL-1
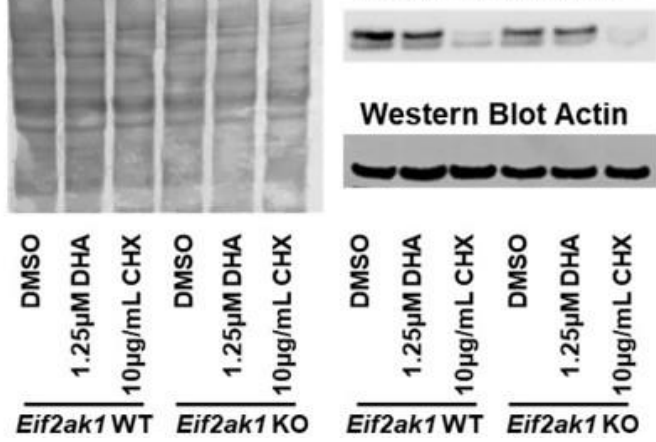

Figure 3-5. HRI is required for DHA-induced MCL-1 repression

(A) BCR-ABL ${ }^{+}$B-ALL cells were treated with DHA for $16 \mathrm{~h}$ and protein expression was determined by immunoblotting with indicated antibodies. (B) Wild-type (WT) or Eif2akl-KO (lacking HRI) BCR-ABL ${ }^{+}$B-ALL cells were treated with DHA for $16 \mathrm{~h}$ and protein expression was determined by immunoblotting with indicated antibodies. (C) $\mathrm{BCR}-\mathrm{ABL}^{+} \mathrm{B}-\mathrm{ALL}$ cells were treated with $1.25 \mu \mathrm{M}$ DHA for $16 \mathrm{~h}$. The integrated stress response inhibitor, ISRIB, was added at the indicated doses for an additional $2 \mathrm{~h}$. Protein expression was determined by immunoblotting with indicated antibodies. (D) Wild-type (WT) or Eif2akl-KO (HRI-deficient) BCR-ABL ${ }^{+}$B-ALL cells were treated with succinylacetone (SA) for $48 \mathrm{~h}$. Protein expression was determined by immunoblotting with indicated antibodies. (E) Wild-type or Eif2akl-KO cells were treated with the indicated drugs for $9 \mathrm{~h}$ and then pulse-labeled with ${ }^{35} \mathrm{~S}$-methionine-cysteine for $1 \mathrm{~h}$. Autoradiography was used to determine new protein synthesis. Cycloheximide (CHX) served as a positive control for translation inhibition. Total protein was determined by Ponceau staining. MCL-1 expression was determined by immunoblotting. (F) Purified HRI protein was incubated with $20 \mu \mathrm{M}$ heme, $20 \mu \mathrm{M}$ DHA, or a combination of the two at room temperature for $30 \mathrm{~min}$. Absorbance was then measured at $420 \mathrm{~nm}$ to detect the presence of a Soret peak which is indicative of protein binding. Data are the average of three experiments and error bars are SEM. Unpaired t-test indicates significance between HRI+Heme vs. HRI+Heme+DHA p $<0.01 * *$. 
treatment (Figure 3-1), thus excluding PERK and the classical ER stress pathway as being responsible for eIF2 $\alpha$ phosphorylation triggered by DHA.

The eIF $2 \alpha$ kinase HRI phosphorylates eIF $2 \alpha$ in erythrocytes to prevent hemoglobin translation when cellular heme levels are limiting [95]. To assess whether $\mathrm{HRI}$ is responsible for MCL-1 repression in BCR-ABL ${ }^{+} \mathrm{B}-\mathrm{ALL}$ cells treated with DHA, we generated Eif2akl-deficient (Eif2ak1 encodes HRI, hereafter referred to as HRIdeficient) $\mathrm{BCR}-\mathrm{ABL}^{+} \mathrm{B}$-ALL cells using CRISPR/Cas9. In contrast to wild-type BCR$\mathrm{ABL}^{+} \mathrm{B}-\mathrm{ALL}$ cells, HRI-deficient BCR-ABL ${ }^{+} \mathrm{B}-\mathrm{ALL}$ cells, failed to induce the expression of either ATF4 or CHOP when treated with DHA (Figure 3-5B). Notably, while DHA treatment in HRI expressing BCR-ABL ${ }^{+} \mathrm{B}-\mathrm{ALL}$ cells triggered the repression of MCL-1 expression, the BCR-ABL ${ }^{+} \mathrm{B}-\mathrm{ALL}$ cells lacking HRI failed to repress MCL-1 expression (Figure 3-5B, E). Furthermore, the addition of ISRIB, a small-molecule inhibitor of the integrated stress response [96], blocked the repression of MCL-1 and attenuated the induction of the integrated stress response (indicated by ATF4) in response to DHA treatment (Figure 3-5C). Although cellular heme depletion is one mechanism of integrated stress response induction, $24 \mathrm{~h}$ of SA treatment was insufficient on its own to activate the integrated stress response; however, culture with SA for 48h resulted in the HRI-dependent induction of MCL-1 repression and activation of the integrated stress response (Figure 3-5D). These data indicate that HRI is required for the DHA induced eIF2 $\alpha$ phosphorylation and activation of the down-stream integrated stress response, which represses MCL-1 expression.

HRI-mediated phosphorylation of eIF2 $\alpha$ represses cap-dependent translation and blocks a wide array of protein synthesis [94]. To confirm whether active HRI is upstream of the MCL-1 translational repression we used metabolic labeling to assess whether loss of HRI attenuates translational inhibition triggered by DHA treatment. HRI wild-type or deficient $\mathrm{BCR}-\mathrm{ABL}^{+} \mathrm{B}-\mathrm{ALL}$ cells were treated with DHA for $9 \mathrm{~h}$ and then pulse-labeled with $100 \mu \mathrm{Ci} / \mathrm{ml}$ of ${ }^{35} \mathrm{~S}$-labeled methionine and cysteine for $1 \mathrm{~h}$. Autoradiography of whole cell lysates from treated cells demonstrated that global translation was repressed in DHA treated control cells; however, the repression of translation was substantially blunted in HRI-deficient cells in response to DHA treatment (Figure 3-5E). As a positive control, culture of both cell types with cycloheximide (CHX) repressed translation in an HRI-independent manner. Western blotting of the treated cells also revealed that MCL-1 was repressed in the control cells, but not the HRI-deficient BCR$\mathrm{ABL}^{+} \mathrm{B}-\mathrm{ALL}$ cells (Figure 3-5E). These data indicate that DHA treatment induces an HRI-dependent inhibition of translation that results in MCL-1 repression.

In erythrocytes, HRI directly binds to heme, which represses kinase activity; however, when heme levels drop, HRI becomes active and phosphorylates eIF2 $\alpha$ [56, 57]. To address how DHA triggers HRI activation, we assessed the HRI-heme interaction using spectroscopy on purified, recombinant proteins. When HRI and heme were incubated together, a Soret peak was detected indicating binding of the two proteins. However, when DHA was incubated with heme and HRI, the Soret peak was lost indicating that DHA addition disrupts the interaction between heme and HRI (Figure 
3-5F). These data indicate that DHA can disrupt the HRI-heme interaction in a cell-free system.

\section{HRI Is Required for the Synergistic Response of DHA and BH3-Mimetics}

Previous studies demonstrated that DHA-induced repression of MCL-1 renders BCR-ABL ${ }^{+} \mathrm{B}-\mathrm{ALL}$ cells susceptible to synergistic cell death induced by the BH3mimetic drug ABT-263 [32]. Since HRI is responsible for mediating the repression of MCL-1 in response to DHA treatment, we assessed whether HRI-deficient BCR-ABL ${ }^{+}$ B-ALL cells would exhibit less synergistic cell death in response to combination treatment of DHA with $\mathrm{BH} 3$-mimetic drugs. In control $\mathrm{BCR}-\mathrm{ABL}^{+} \mathrm{B}-\mathrm{ALL}$ cells DHA synergized with both BH3-mimetic drugs ABT-199 and ABT-263 as evidenced by response surface modeling (Figure 3-6A). However, the loss of HRI from BCR-ABL ${ }^{+}$ B-ALL cells substantially blunted the synergistic killing between DHA and both BH3mimetics, indicating that loss of HRI attenuated the synergism between DHA and BH3mimetics because MCL-1 was not repressed (Figure 3-6A). Although synergy with DHA and BH3-mimetics was diminished by loss of HRI, there was still sensitization to BH3-mimetics indicating that DHA has HRI-independent effects as well. Overexpression of MCL-1 also attenuated the synergism between DHA and BH3-mimetics, indicating that the synergy depends on the expression levels of MCL-1 (Figure 3-6B). DHA treatment did not induce significant changes in expression of other anti-apoptotic BCL-2 family members, including BCL-2 or BCL-XL; however, the pro-apoptotic proteins NOXA and PUMA, which are known to bind to MCL-1, were induced (Figure 3-5A). These data indicate that the synergy between DHA and BH3-mimetics depends mainly on changes in MCL-1 expression and not on other anti-apoptotic proteins.

\section{Direct Activation of HRI Represses MCL-1 and Synergizes with BH3-Mimetics}

DHA repressed MCL-1 in an HRI-dependent manner, so we asked whether a small molecule activator of HRI, BTdCPU [97], could also induce the repression of MCL-1. Similar to DHA treatment, when BCR-ABL ${ }^{+}$B-ALL cells were treated with BTdCPU MCL-1 expression was repressed whereas BCL-XL expression was unaffected (Figure 3-7A). Consistently, much less MCL-1 repression was induced by BTdCPU treatment in the HRI-deficient leukemic cells indicating that BTdCPU induced an HRIdependent repression of MCL-1 (Figure 3-7A). Additionally, BTdCPU triggered the integrated stress response and induced the downstream targets CHOP and ATF4 in wildtype, but not HRI-deficient BCR-ABL ${ }^{+} \mathrm{B}-\mathrm{ALL}$ cells (Figure 3-7A). These data indicate that BTdCPU can activate HRI and induce MCL-1 repression.

Since BTdCPU can induce MCL-1 repression in an HRI-dependent manner, we assessed whether it can synergize with $\mathrm{BH} 3$-mimetics in killing BCR-ABL ${ }^{+} \mathrm{B}-\mathrm{ALL}$ cells. When BTdCPU was combined with either ABT-199 or ABT-263 it induced a synergistic cell death response in wild-type BCR-ABL ${ }^{+} \mathrm{B}-\mathrm{ALL}$ cells (Figure 3-7B). However, in HRI-deficient BCR-ABL ${ }^{+} \mathrm{B}-\mathrm{ALL}$ cells the synergistic combination of BTdCPU with 
Figure 3-6. HRI is required for the synergistic response of DHA and BH3mimetics

(A) WT or Eif2akl-KO BCR-ABL ${ }^{+}$B-ALL cells were treated with ABT-263 or ABT$199(0,40,80,160 \mathrm{nM})$ alone or in combination with the indicated concentrations of DHA for $24 \mathrm{~h}$. Viable cells were measured using Annexin- $\mathrm{V}$ and propidium iodide staining. Data are the average of three experiments and error bars are SEM. Two-way ANOVA with Bonferroni multiple comparison indicates significance $\mathrm{p}<0.0001 * * * *$ between the DHA alone (0 nM ABT-263 or ABT-199) and $160 \mathrm{nM}$ ABT-263 or ABT199 at indicated doses of DHA. The combination of DHA+ABT-263 showed a statistically less synergistic response in Eif2akl-KO $(\alpha=3.15, \mathrm{p}=5.67 \mathrm{e}-06)$ as compared to wild-type $(\alpha=5.41, \mathrm{p}=7.5 \mathrm{e}-08) \mathrm{BCR}-\mathrm{ABL}^{+} \mathrm{B}-\mathrm{ALL}$ cells $\left(\mathrm{p}<10^{-5}\right)$. The combination of DHA+ABT-199 showed a significantly less synergistic response in Eif2akl-KO $(\alpha=5.4$, $\mathrm{p}=1.16 \mathrm{e}-83)$ as compared to wild-type $(\alpha=7.96, \mathrm{p}=2.7 \mathrm{e}-32) \mathrm{BCR}-\mathrm{ABL}^{+} \mathrm{B}-\mathrm{ALL}$ cells $(\mathrm{p}<0.057)$. (B) MCL-1 WT or overexpressing (OE) BCR-ABL ${ }^{+} \mathrm{B}-\mathrm{ALL}$ cells were treated with ABT-263 or ABT-199 $(0,40,80,160 \mathrm{nM})$ alone or in combination with the indicated concentrations of DHA for 24h. Viable cells were measured using Annexin-V and propidium iodide staining. Data are the average of four experiments and error bars are SEM. Two-way ANOVA with Bonferroni multiple comparison indicates significance $\mathrm{p}<0.05^{*}, \mathrm{p}<0.001 * * *, \mathrm{p}<0.0001 * * * *$ between the DHA alone $(0 \mathrm{nM}$ ABT263 or ABT-199) and $160 \mathrm{nM} \mathrm{ABT-263}$ or ABT-199 at indicated doses of DHA. The combination of DHA+ABT-263 showed a statistically less synergistic response in MCL$1^{\mathrm{OE}}$ cells $(\alpha=24.1, \mathrm{p}=6.63 \mathrm{e}-62)$ as compared to MCL- ${ }^{\mathrm{WT}}(\alpha=29.8, p=2.05 \mathrm{e}-47)$ BCR$\mathrm{ABL}^{+} \mathrm{B}-\mathrm{ALL}$ cells $(\mathrm{p}=0.00027)$. The combination of DHA+ABT-199 showed a significantly less synergistic response in MCL- ${ }^{\mathrm{OE}}(\alpha=3.96, p=9.38 \mathrm{e}-75)$ as compared to MCL- ${ }^{\mathrm{WT}}(\alpha=4.84, \mathrm{p}=1.37 \mathrm{e}-39)$ BCR-ABL ${ }^{+} \mathrm{B}-\mathrm{ALL}$ cells $(\mathrm{p}=.0013)$. 
A.

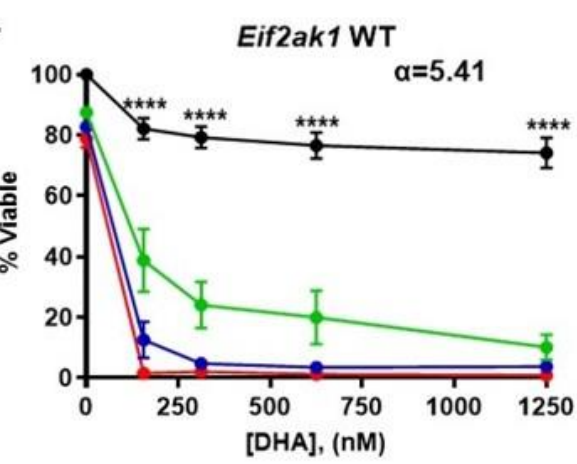

Eif2ak1 WT

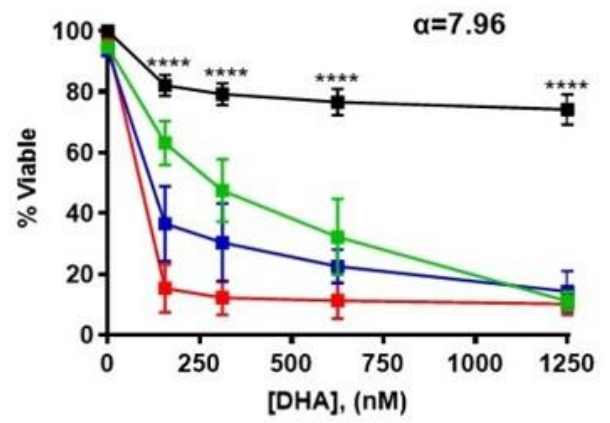

B.
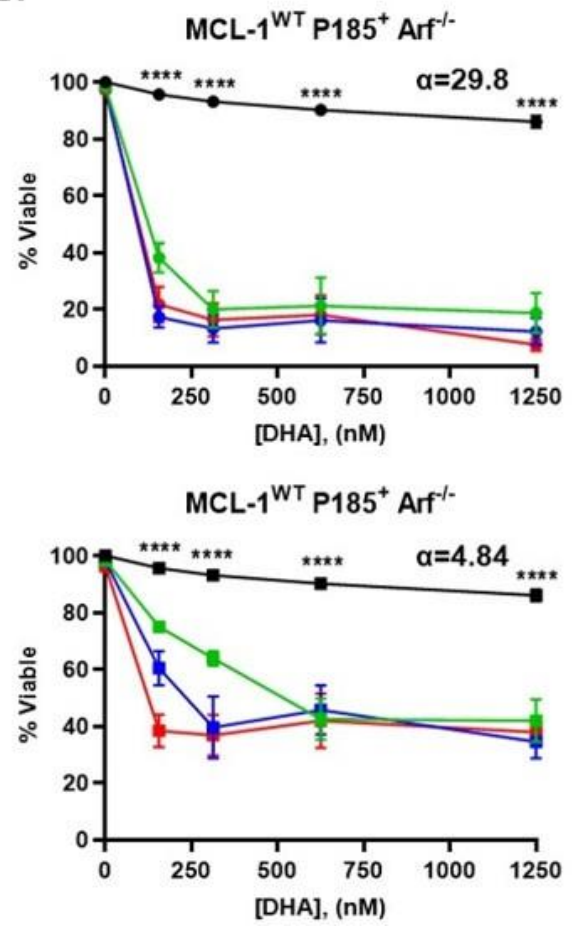

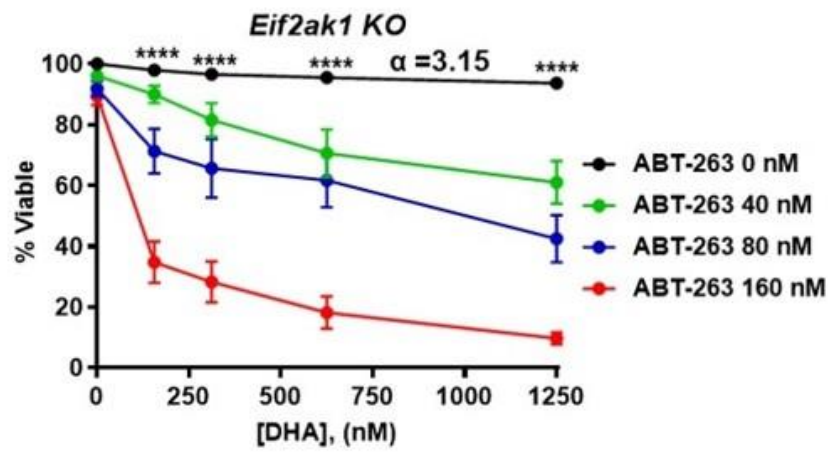

Eif2ak1 KO
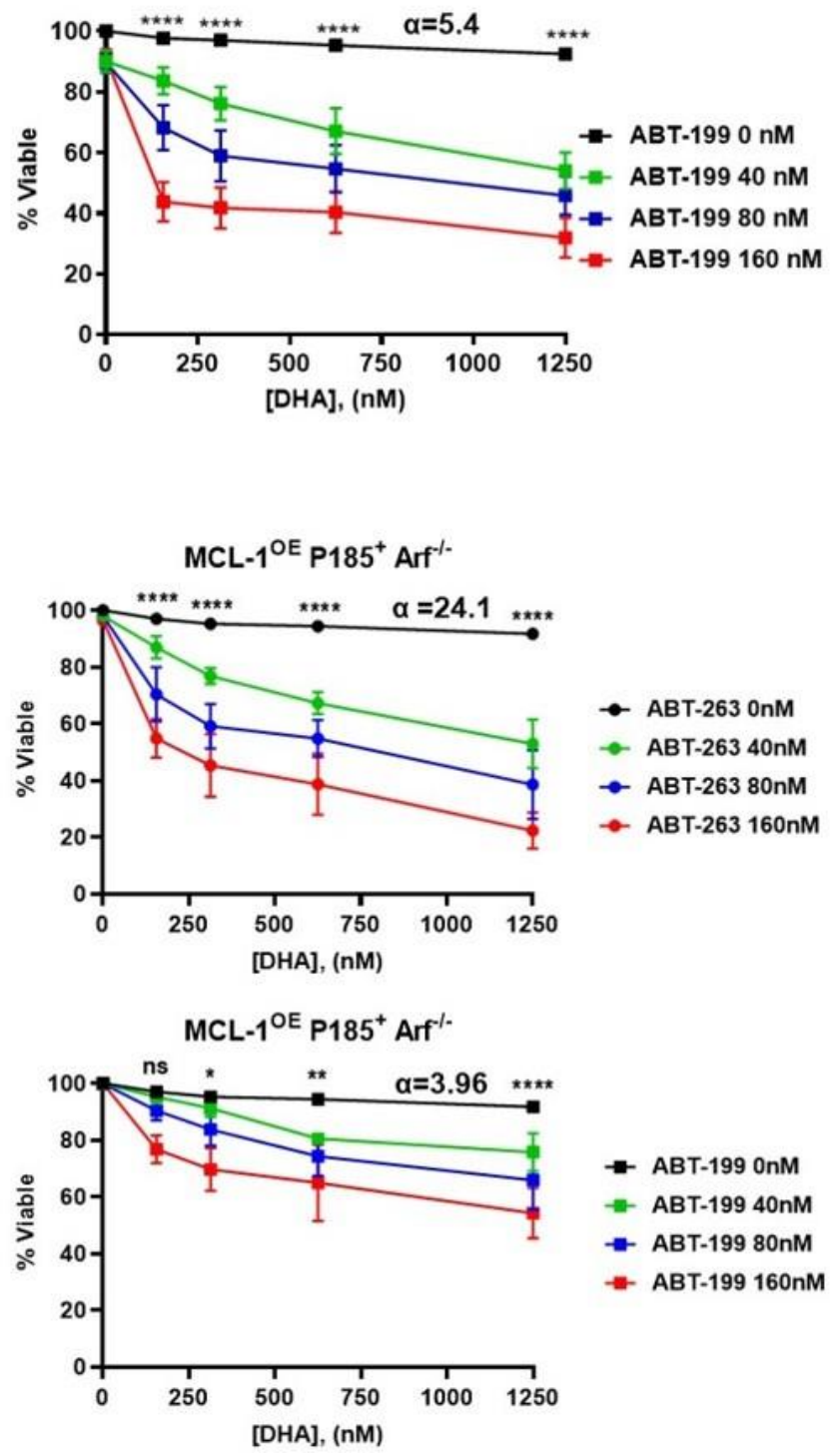


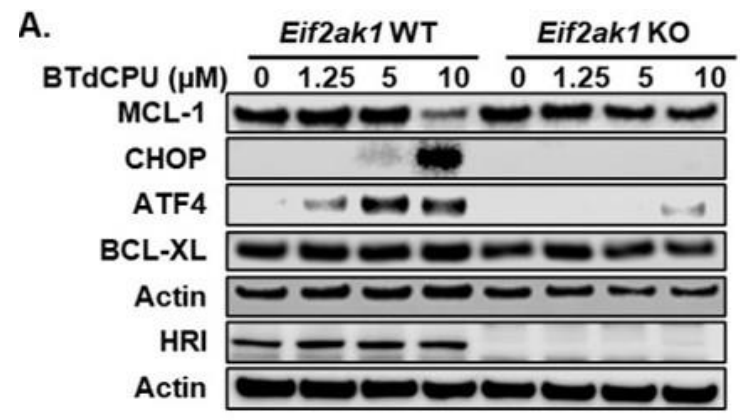

B.
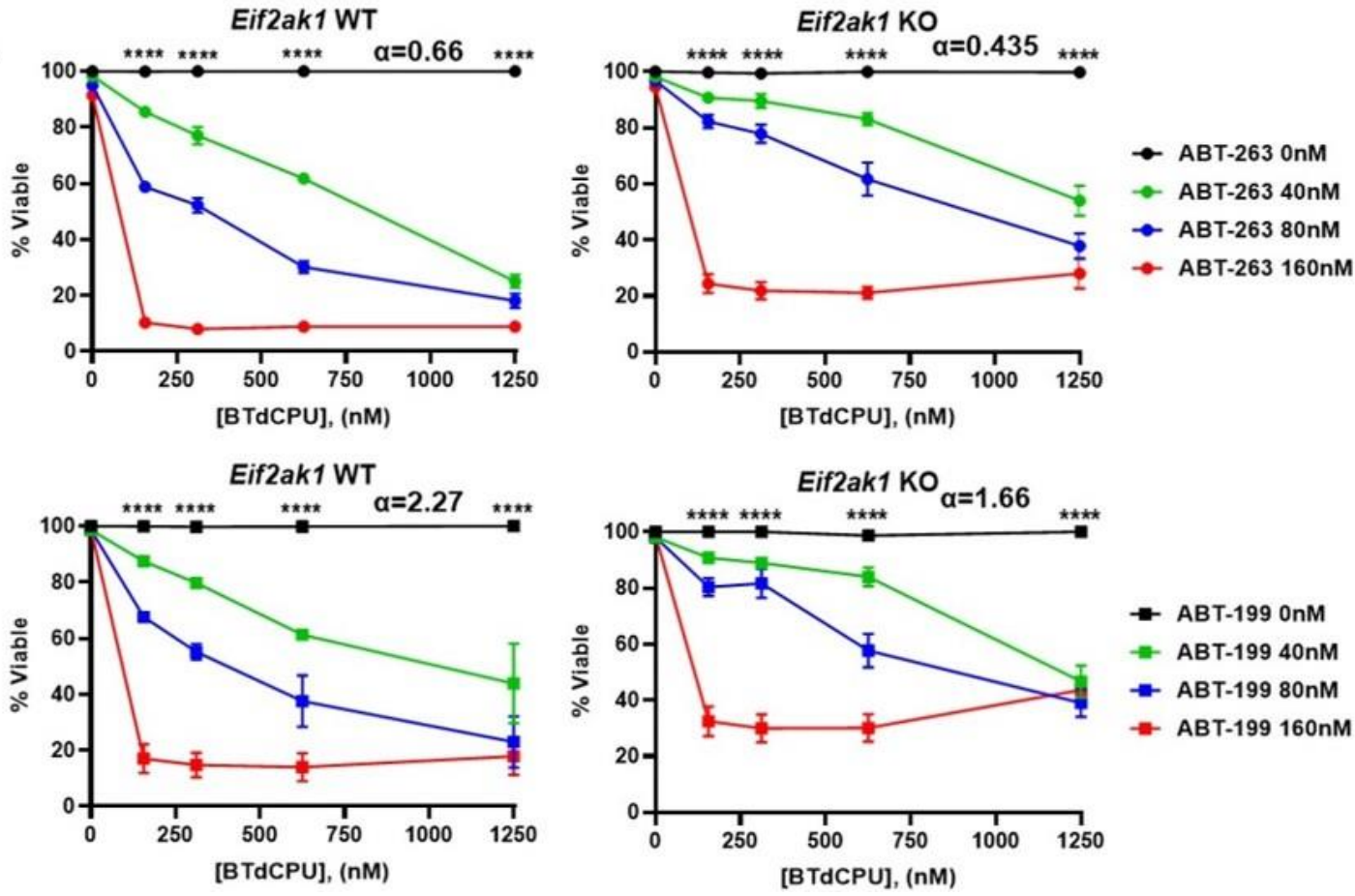

Figure 3-7. Direct activation of HRI represses MCL-1 and synergizes with BH3mimetics

(A) Wild-type (WT) or Eif2akl-KO (HRI-deficient) BCR-ABL ${ }^{+}$B-ALL cells were treated with BTdCPU for $16 \mathrm{~h}$ and protein expression was determined by immunoblotting with indicated antibodies. (B) WT or Eif2akl-KO BCR-ABL ${ }^{+} \mathrm{B}-\mathrm{ALL}$ cells were treated with ABT-263 or ABT-199 $(0,40,80,160 \mathrm{nM})$ alone or in combination with the indicated concentrations of BTdCPU for $24 \mathrm{~h}$. Viable cells were measured using Annexin- $V$ and propidium iodide staining. Data are the average of three experiments and error bars are SEM. Two-way ANOVA with Bonferroni multiple comparison indicates significance $\mathrm{p}<0.0001 * * * *$ between the BTdCPU alone ( $0 \mathrm{nM}$ ABT-263 or ABT-199) and $160 \mathrm{nM}$ ABT-263 or ABT-199 at indicated doses of BTdCPU. The combination of BTdCPU+ABT-263 showed a statistically less synergistic response in Eif2akl-KO $(\alpha=0.435, p=1.16 \mathrm{e}-12)$ as compared to wild-type $(\alpha=0.66, \mathrm{p}=2.826 \mathrm{e}-13) \mathrm{BCR}_{\mathrm{ABL}} \mathrm{A}^{+} \mathrm{B}-$ ALL cells $(\mathrm{p}<0.05)$. The combination of BTdCPU+ABT-199 showed a statistically less synergistic response in Eif2akl-KO $(\alpha=1.66, \mathrm{p}=3.79 \mathrm{e}-18)$ as compared to wild-type $(\alpha=2.27, \mathrm{p}=1.75 \mathrm{e}-26) \mathrm{BCR}-\mathrm{ABL}^{+} \mathrm{B}-\mathrm{ALL}$ cells $(\mathrm{p}<0.05)$. 
both BH3-mimetics was significantly attenuated (Figure 3-7B). Although synergy with BTdCPU and BH3-mimetics was diminished by loss of HRI, there was still sensitization observed indicating that BTdCPU has HRI-independent effects. These data indicate that the activation of HRI by BTdCPU can induce synergistic leukemia cell killing in our murine cell model of BCR-ABL ${ }^{+} \mathrm{B}-\mathrm{ALL}$.

\section{HRI Activation Synergizes with BH3-Mimetics in ALL Cell Lines}

To assess whether activation of HRI can synergize with BH3-mimetic agents in human $\mathrm{Ph}^{+}$leukemia cells, BV-173 cells, were cultured with either DHA or BTdCPU. Similar to our observations in mouse BCR-ABL ${ }^{+} \mathrm{B}-\mathrm{ALL}$ cells, treatment of human leukemia cells with DHA or BTdCPU induced eIF2 $\alpha$ phosphorylation, expression of CHOP and ATF4, and repression of MCL-1 without altering BCL-XL expression (Figure 3-8A). In murine BCR-ABL ${ }^{+} \mathrm{B}-\mathrm{ALL}$ cells, the repression of MCL-1 triggered by either DHA or BTdCPU required the HRI-mediated activation of the integrated stress response; therefore, we sought to determine whether human $\mathrm{Ph}^{+}$acute leukemia cells were similarly HRI-dependent. To this aim, EIF2AK1 (encoding HRI) was ablated in $\mathrm{BV}-173 \mathrm{Ph}^{+}$leukemia cell lines by CRISPR/Cas9 and the cells were subjected to treatment with DHA, BTdCPU, or thapsigargin (an inducer of the classical ER stress pathway) [98]. As in mouse BCR-ABL ${ }^{+} \mathrm{B}-\mathrm{ALL}$ cells, loss of HRI expression prevented the repression of MCL-1 expression in cells cultured with DHA or BTdCPU and blocked the repression of ATF4 and CHOP (Figure 3-8B). In contrast, thapsigargin treatment still repressed MCL-1 and induced ATF4 and CHOP in BV-173 cells lacking HRI, confirming that the ER stress pathway does not depend on HRI (Figure 3-8B). These data indicate that activation of HRI-mediated eIF2 $\alpha$ phosphorylation pathway, by either DHA or BTdCPU treatment, can cause MCL-1 repression and therefore should be able to synergize with $\mathrm{BH} 3$-mimetics in human $\mathrm{Ph}^{+}$leukemia cells. Consistent with these findings, when BV-173 cells were cultured with either DHA or BTdCPU and combined with BH3-mimetics (ABT-199 or ABT-263), the combined treatments were significantly synergistic with both BH3-mimetics, but in BV-173 cells lacking HRI expression the synergism was significantly reduced (Figure 3-9). Similar synergism between either DHA or BTdCPU and BH3-mimetics were obtained in human TOM1, OP-1, and SUP$\mathrm{B} 15 \mathrm{Ph}^{+}$leukemia cell lines treated in culture (Figure 3-10).

We next wanted to extend our findings to other poor prognosis subtypes of ALL. To achieve this aim, we tested DHA and BTdCPU in a mouse cell line expressing fusion oncoprotein drivers of Ph-like ALL. Mouse Ba/F3 cells stably expressing human PAX5$J A K 2$, RCSD1-ABL1, or RCSD1-ABL2 fusions were treated with DHA or BTdCPU to determine if MCL-1 was repressed [78]. Consistent with $\mathrm{Ph}^{+}$leukemia cell lines, we found that MCL-1 expression was repressed in response to DHA or BTdCPU in $\mathrm{Ba} / \mathrm{F} 3$ cells expressing the Ph-like fusions (Figure 3-11). Additionally, eIF2 $\alpha$ phosphorylation was induced, ATF4 and CHOP were expressed, and BCL-XL protein was unaffected by treatment with either DHA or BTdCPU. Since MCL-1 was repressed in the $\mathrm{Ba} / \mathrm{F} 3$ cells modeling Ph-like ALL, we next investigated whether there was a synergistic effect when DHA or BTdCPU was combined with BH3-mimetics. As expected, synergy was 

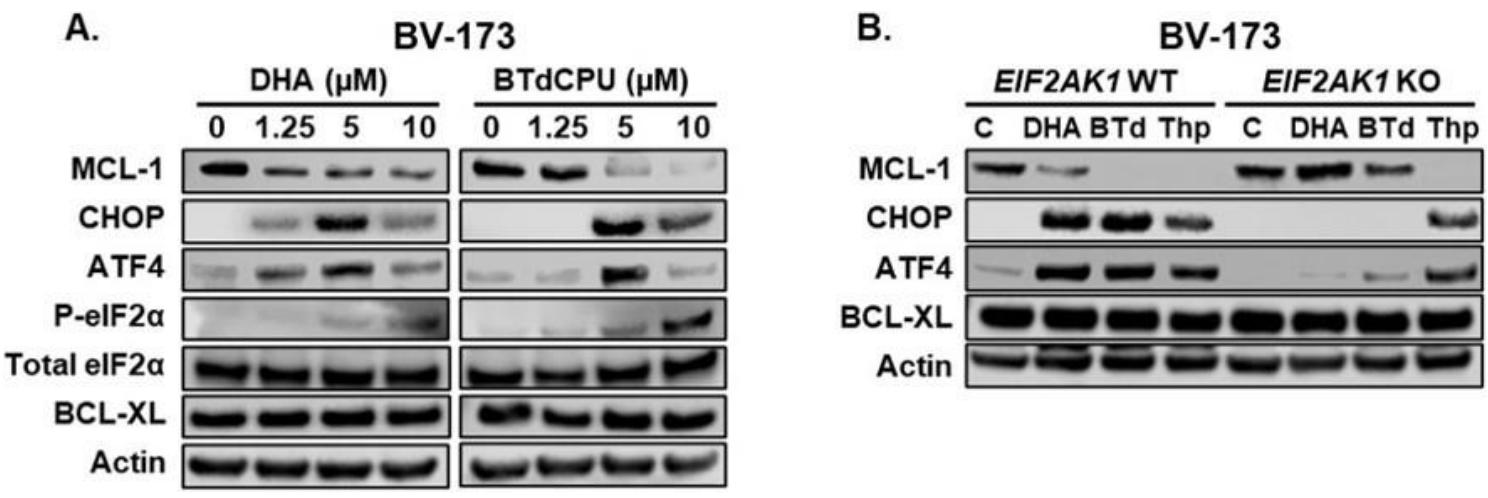

Figure 3-8. HRI activation represses MCL-1 in human $\mathrm{Ph}^{+} \mathrm{BV}-173$ cells

(A) Human $\mathrm{Ph}^{+} \mathrm{BV}-173$ cells were treated with the indicated concentrations of DHA or BTdCPU for $24 \mathrm{~h}$ and protein expression was determined by western blotting. (B) Wildtype (WT) or a pool of EIF2AK1-KO (lacking HRI) BV-173 cells were treated with DMSO (Control, C), DHA $(5 \mu \mathrm{M})$, BTdCPU (BTd, $5 \mu \mathrm{M})$, or Thapsigargin (Thp, 100 $\mathrm{nM})$ for $24 \mathrm{~h}$ and protein expression was determined by western blotting. 
Figure 3-9. HRI activation synergizes with BH3-mimetics in human $\mathrm{Ph}^{+} \mathrm{BV}-173$ cells

WT or EIF2AK1-KO BV-173 cells were treated with ABT-263 $(0,5,10,20 \mathrm{nM})$ or ABT-199 $(0,2.5,5,10 \mathrm{nM})$ alone or in combination with the indicated concentrations of (A) DHA or (B) BTdCPU for 24h. Viable cells were measured using Annexin-V and propidium iodide staining. Data are the average of 3 experiments and error bars are SEM. Two-way ANOVA with Bonferroni multiple comparison indicates significance $\mathrm{p}<0.0001 * * * *$ between DHA or BTdCPU alone (0 nM ABT-263 or ABT-199) and 20 nM ABT-263 or 10 ABT-199 at indicated doses of DHA or BTdCPU. The combination of DHA+ABT-263 showed significantly less synergistic response in BV-173 EIF2AK1$\mathrm{KO}(\alpha=23, \mathrm{p}=1.05 \mathrm{e}-03)$ as compared to $\mathrm{BV}-173$ wild-type $(\alpha=94.2, \mathrm{p}=1.66 \mathrm{e}-08)$ cells $(\mathrm{p}<0.003)$. The combination of DHA+ABT-199 showed a less synergistic response in BV-173 EIF2AKI-KO $(\alpha=1.58, \mathrm{p}=2.54 \mathrm{e}-01)$ as compared to BV-173 wild-type $(\alpha=69.1$, $\mathrm{p}=4.49 \mathrm{e}-08)$ cells $(\mathrm{p}<0.003)$. The combination of BTdCPU+ABT-199 showed a less synergistic response in EIF $2 A K 1-\mathrm{KO}(\alpha=3.33$, $\mathrm{p}=3.05 \mathrm{e}-02)$ as compared to wild-type $(\alpha=12.3, \mathrm{p}=5.53 \mathrm{e}-06)$ cells $(\mathrm{p}<0.003)$. 
A.

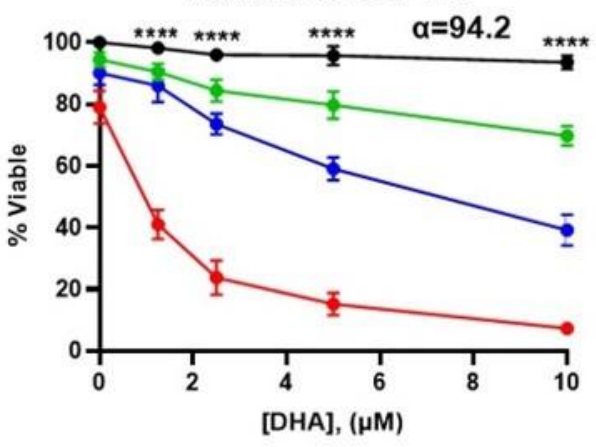

EIF2AK1 WT BV-173

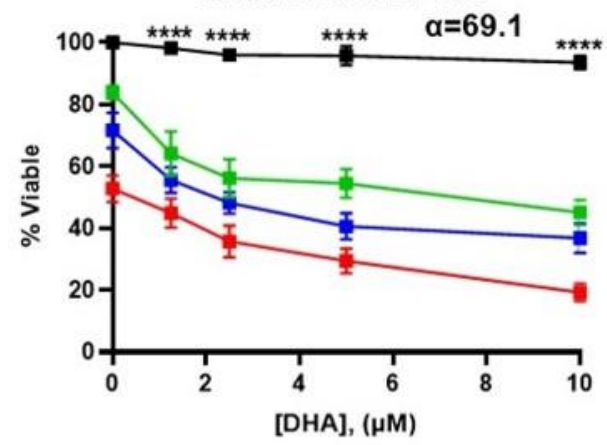

B.

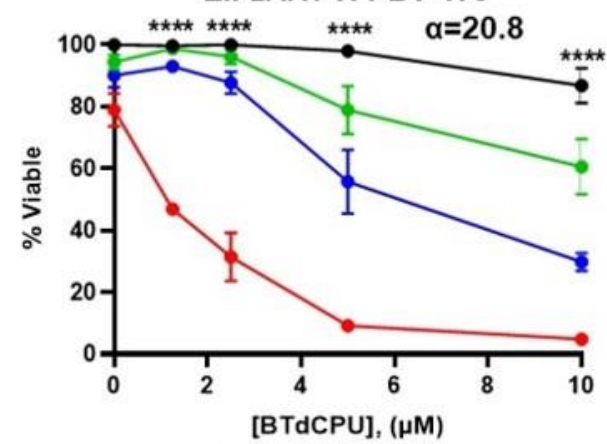

EIF2AK1 WT BV-173

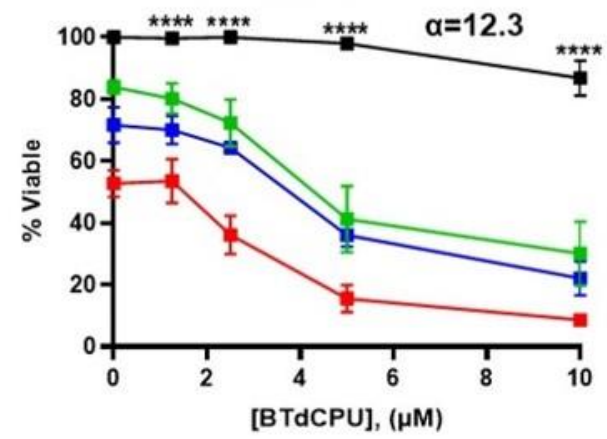

EIF2AK1 KO BV-173

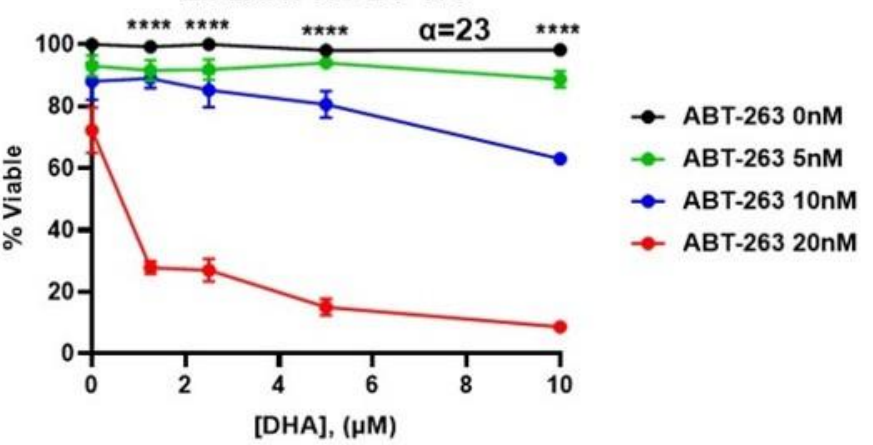

EIF2AK1 KO BV-173

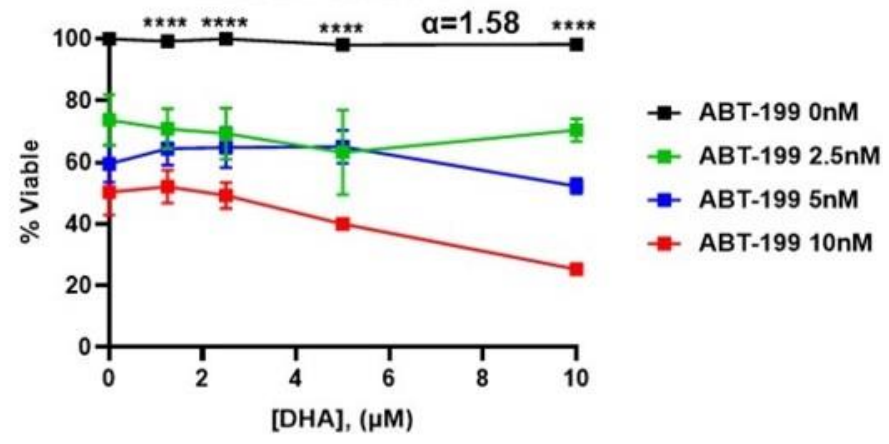

EIF2AK1 KO BV-173

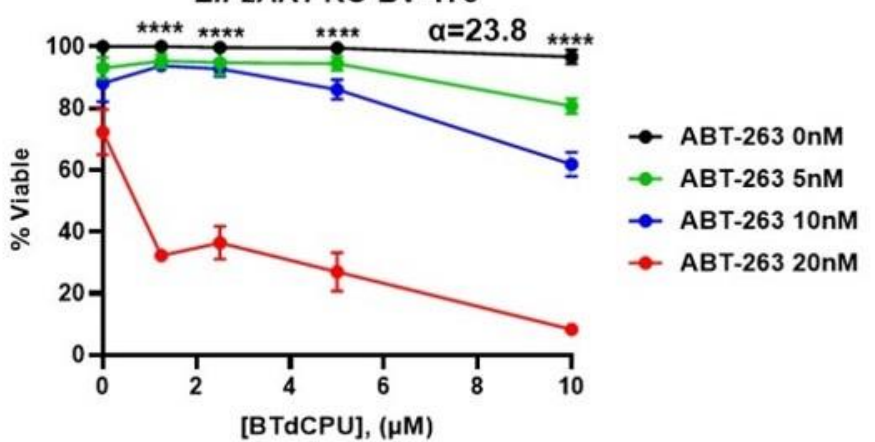

EIF2AK1 KO BV-173

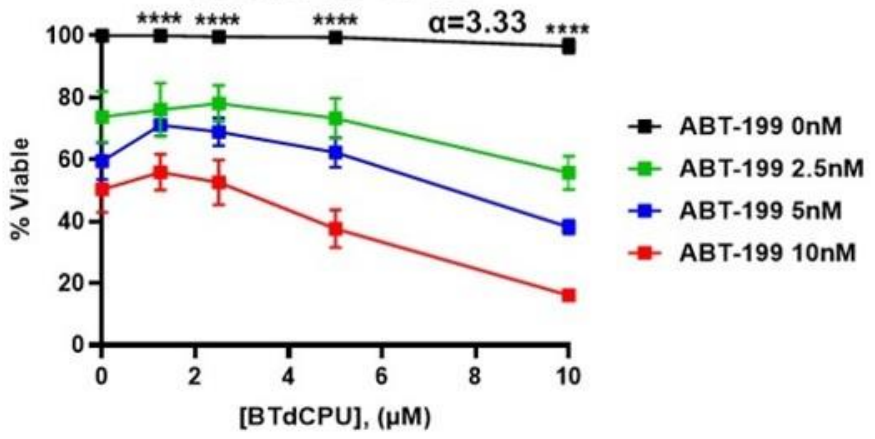


Figure 3-10. HRI activation synergizes with $\mathrm{BH3}$-mimetics in human $\mathrm{Ph}^{+}$cell lines Human $\mathrm{Ph}^{+}$ALL cell lines (A) Tom1, (B) OP-1, and (C) SUP-B15 were treated with the indicated concentrations of ABT-263 or ABT-199 combined with DHA or BTdCPU for $24 \mathrm{~h}$ and viability was measure by Annexin- $\mathrm{V}$ and propidium iodide staining. Two-way ANOVA with Bonferroni multiple comparison indicates significance $\mathrm{P}<0.0001 * * * *$ between DHA or BTdCPU alone (0 nM ABT-263 or ABT-199) and $80 \mathrm{nM}$ ABT-263 or $160 \mathrm{nM}$ ABT-199 (for A and B) and $20 \mathrm{nM} \mathrm{ABT-263} \mathrm{or} 5 \mathrm{nM} \mathrm{ABT-199}$ (for C) at indicated doses of DHA or BTdCPU. Response surface modeling was used to assess synergy of these drug combinations, indicated by an $\alpha$ value. 
A.
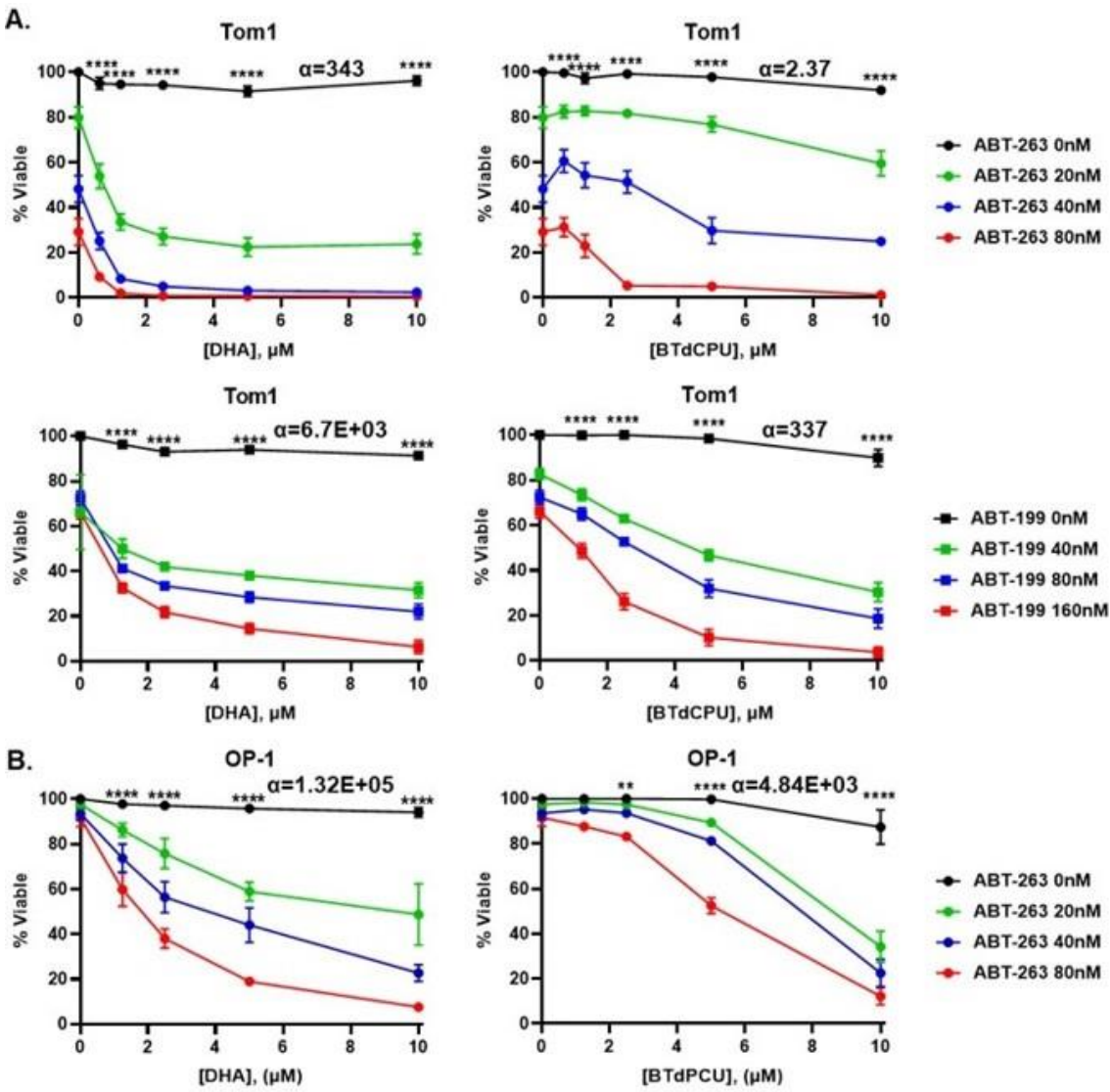

OP-1

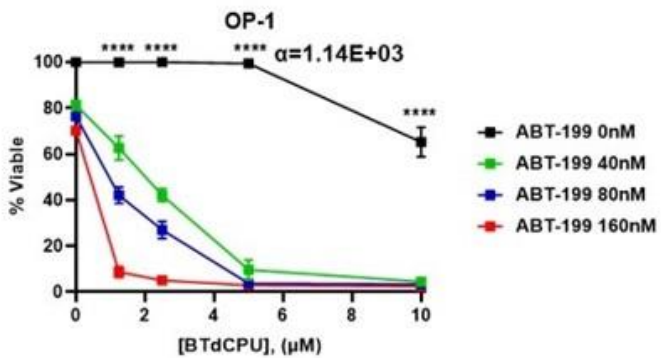

C.

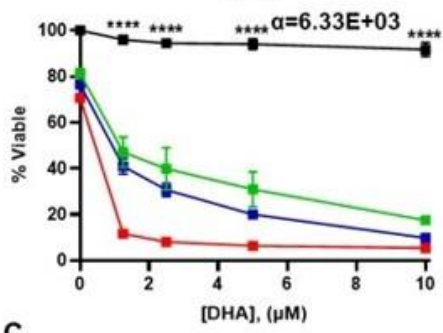

SUP-B15

SUP-B15
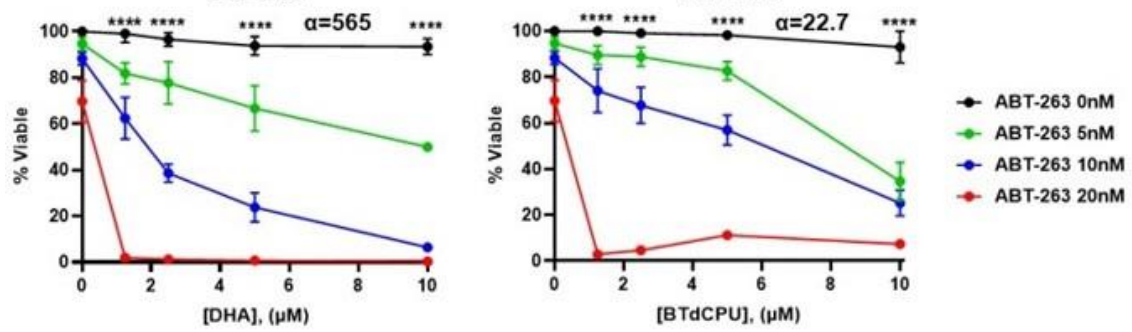

SUP-B15
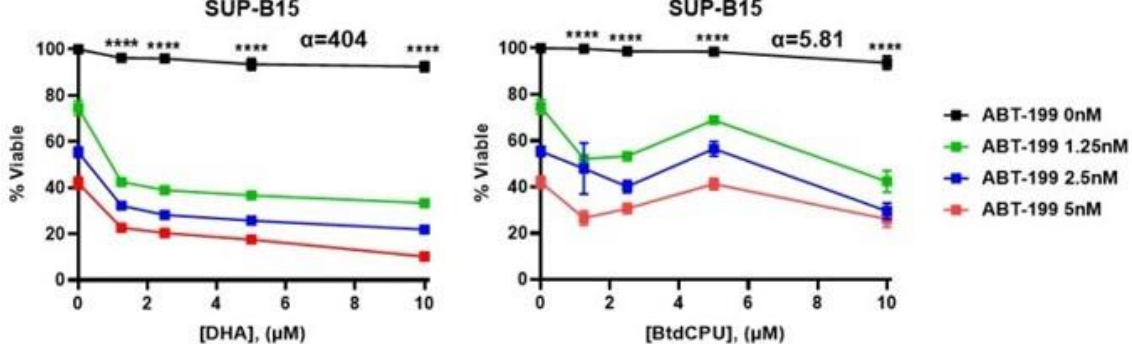

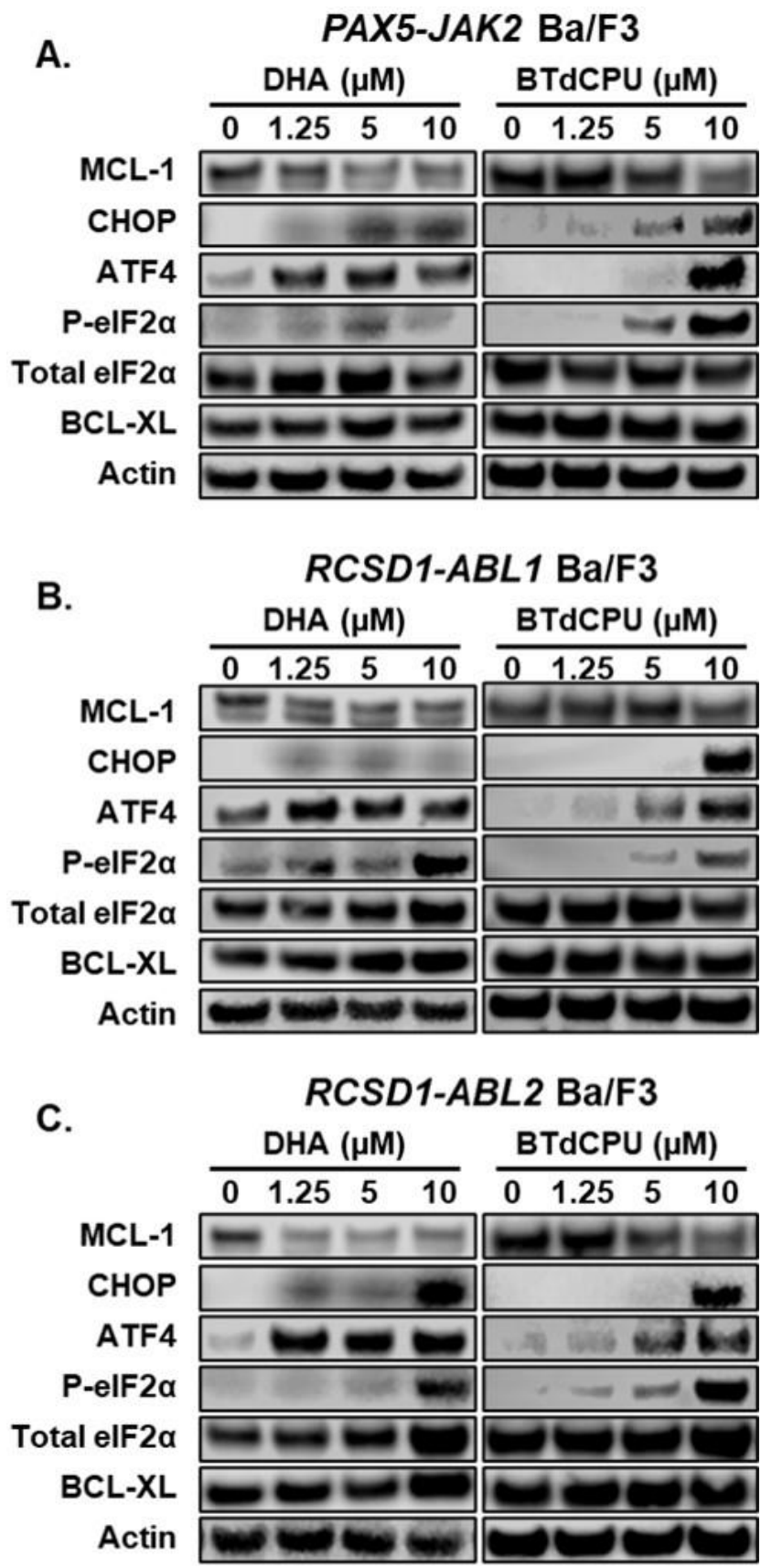

Figure 3-11. HRI activation represses MCL-1 and induces the integrated stress response in Ph-like ALL cell lines

$\mathrm{Ba} / \mathrm{F} 3$ cells expressing the (A) PAX5-JAK2, (B) RCSD1-ABL1, or (C) RCSD1-ABL2 fusions were treated with the indicated doses of DHA or BTdCPU for $9 \mathrm{~h}$ and protein expression was determined by immunoblotting with indicated antibodies. 
observed in the $\mathrm{Ba} / \mathrm{F} 3$ cells expressing the PAX5-JAK2, RCSD1-ABL1, and RCSD1-ABL2 fusions when DHA or BTdCPU was combined with BH3-mimetics (Figure 3-12).

\section{HRI Activation Synergizes with BH3-Mimetics in Primary Patient-Derived Xenografts of Human B-ALL}

To confirm whether DHA or BTdCPU can lead to synergistic responses when combined with ABT-199 or ABT-263 BH3-mimetics in primary patient leukemia, we took advantage of primary patient derived xenografts (PDXs) established from patients with $\mathrm{Ph}^{+}$or EBF1-PDGFRB expressing Ph-like B-ALL. PDX cells isolated from recipient mice were cultured overnight with either DHA or BTdCPU and either ABT-199 or ABT-263 BH3-mimetics. Similar to $\mathrm{Ph}^{+}$human cell lines, the combination of either $\mathrm{BH} 3$-mimetic agent synergized with both DHA and BTdCPU in $\mathrm{Ph}^{+} \mathrm{B}-\mathrm{ALL}$ patient derived xenograft samples (Figure 3-13A). Consistently, EBF1-PDGFRB Ph-like PDX cells also responded synergistically to the combination of DHA or BTdCPU with either BH3-mimetic (Figure 3-13B). Additionally, in $\mathrm{Ph}^{+}$and $\mathrm{Ph}$-like PDX cells treated with DHA or BTdCPU MCL-1 was repressed and ATF4 was induced (Figure 3-13C, D). These data indicate that primary patient leukemic cells can respond to either DHA or BTdCPU-induced synergism with BH3-mimetics agents in culture.

\section{HRI Activation Represses MCL-1 and Synergizes with BH3-Mimetics in vivo}

Since combining HRI activation by BTdCPU synergized with BH3-mimetics in cultured mouse and human leukemia, we hypothesized that combining BTdCPU with ABT-263 could prolong survival of mice bearing BCR-ABL ${ }^{+} \mathrm{B}-\mathrm{ALL}$. To this aim, C57/BL6 mice were injected with BCR-ABL ${ }^{+} \mathrm{B}-\mathrm{ALL}$ cells followed by treatment on day 5 after transplant with either vehicle, BTdCPU (400 mg/kg), ABT-263 (100 mg/kg), or a combination of BTdCPU and ABT-263. Treatments continued daily for 14 days during which mice were monitored. Recipient mice receiving vehicle or either BTdCPU or ABT-263 alone exhibited rapidly progressing leukemia and all mice from these cohorts required euthanasia within 13 days after leukemia injection due to progressive leukemia irrespective of treatment regimen. In contrast, recipient mice treated with the combination of BTdCPU and ABT-263 for 14 days exhibited significantly prolonged survival (Figure 3-14A). Complete blood counts from peripheral blood on day 9 revealed that mice receiving vehicle or BTdCPU alone had higher white blood cell counts (WBCs) whereas those mice receiving the combination treatment had statistically lower WBCs than vehicle treated recipients (Figure 3-14B). These data indicated that the combination of BTdCPU and ABT-263 reduced the leukemia burden in recipient mice.

Mechanistically, BTdCPU triggered the repression of MCL-1 in cultured human and mouse leukemia. We thus investigated whether BTdCPU could induce the repression of MCL-1 expression in mice by treating leukemia bearing recipient mice daily with BTdCPU. After 8 days of treatment the bone marrow was isolated, lysed, and assessed for protein expression by immunoblot analyses. The expression of MCL-1 in 
Figure 3-12. HRI activation synergizes with BH3-mimetics in Ph-like cell lines (A) PAX5-JAK2, (B) RCSD1-ABL1, or (C) RCSD1-ABL2 expressing Ba/F3 cells were treated with the indicated drugs for $24 \mathrm{~h}$. Viable cells were measured using Annexin-V and propidium iodide staining. Data are the average of three experiments and error bars are SEM. Two-way ANOVA with Bonferroni multiple comparison indicates significance $\mathrm{P}<0.0001 * * * *$ between DHA or BTdCPU alone ( $0 \mathrm{nM} \mathrm{ABT}-263$ or ABT$199)$ and $160 \mathrm{nM}$ ABT-263 or ABT-199 at indicated doses of DHA or BTdCPU.

Response surface modeling was used to assess synergy of these drug combinations, indicated by an $\alpha$ value. 
A.
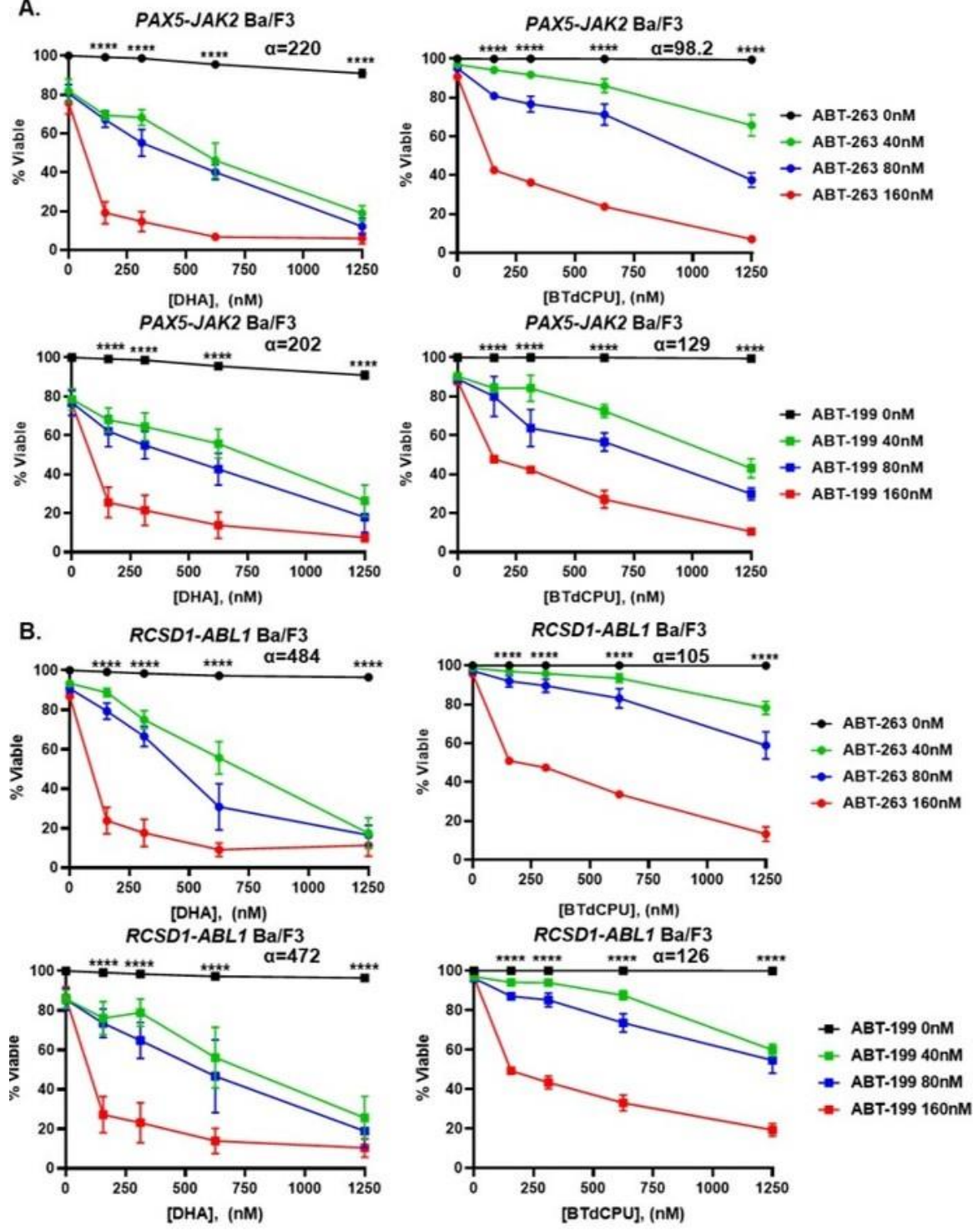

C. RCSD1-ABL2 Ba/F3
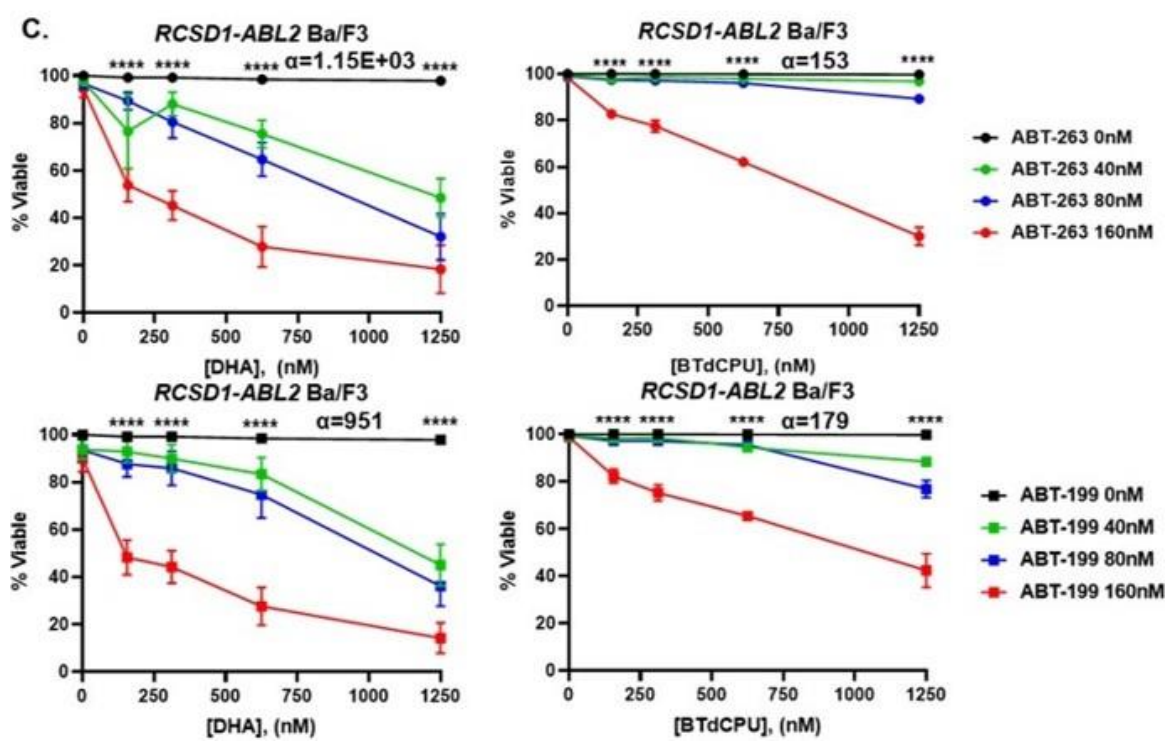
Figure 3-13. HRI activation synergizes with BH3-mimetics and represses MCL-1 in PDX ALL cells

(A) $\mathrm{Ph}^{+} 10940$ or (B) EBF1-PDGFRB Ph-like patient derived xenograft (PDX) cells were treated with the indicated concentrations of ABT-263 or ABT-199 alone or in

combination with DHA or BTdCPU for 16h. Viable cells were measured using Annexin$\mathrm{V}$ and propidium iodide staining. Data are the average of four experiments and error bars are SEM. Two-way ANOVA with Bonferroni multiple comparison indicates significance $\mathrm{P}<0.0001 * * * *$ between DHA or BTdCPU alone ( $0 \mathrm{nM} \mathrm{ABT}-263$ or ABT$199)$ and (40 nM ABT-263 or ABT-199) (for A) or (10 nM ABT-263 or ABT-199) at indicated doses of DHA or BTdCPU (for B). (A) The combination of DHA+ABT-263 or ABT-199 showed synergistic response in $\mathrm{Ph}^{+} \mathrm{PDX}$ cells $(\alpha=1.3, \mathrm{p}=2.11 \mathrm{e}-08)$ and $(\alpha=1.28, p=1.15 \mathrm{e}-07)$ respectively. The combination of BTdCPU+ABT-263 or ABT-199 showed synergistic response in $\mathrm{Ph}^{+} \mathrm{PDX}$ cells $(\alpha=0.672, \mathrm{p}=9.34 \mathrm{e}-03)$ and $(\alpha=0.98$, $\mathrm{p}=2.45 \mathrm{e}-05$ ) respectively. (B) The combination of DHA+ABT-263 or ABT-199 showed synergistic response in Ph -like cells $(\alpha=0.739, p=6.89 \mathrm{e}-10)$ and $(\alpha=0.629, \mathrm{p}=1.66 \mathrm{e}-12)$ respectively. The combination of BTdCPU+ABT-263 orABT-199 showed synergistic response in Ph -like cells $(\alpha=0.432, p=1.37 \mathrm{e}-04)$ and $(\alpha=0.18, \mathrm{p}=5.69 \mathrm{e}-04)$ respectively. (C) $\mathrm{Ph}^{+} 10940$ or (D) Ph-like EBF1-PDGFRB cells were treated with the indicated concentrations of DHA or BTdCPU for 16h and protein expression was determined by western blotting. 
A.

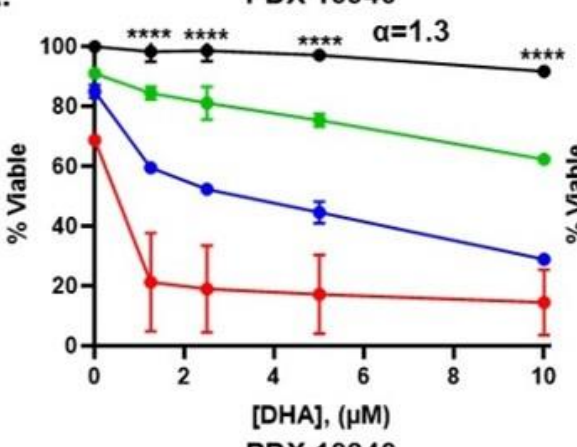

PDX 10940

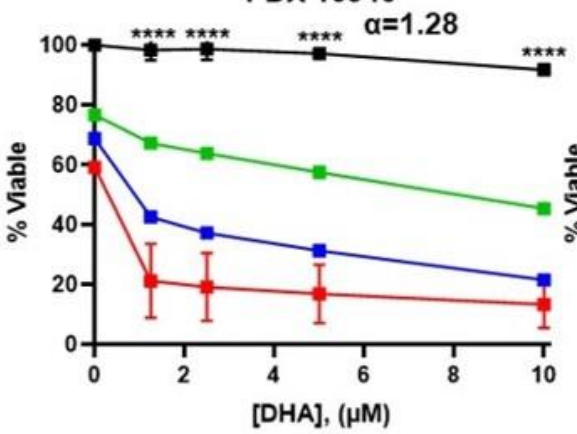

B.

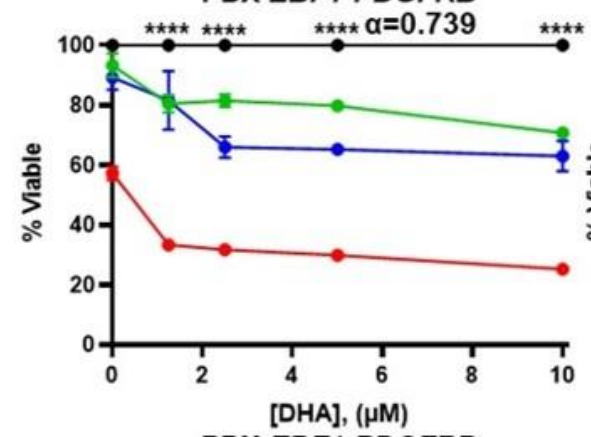

PDX EBF1-PDGFRB

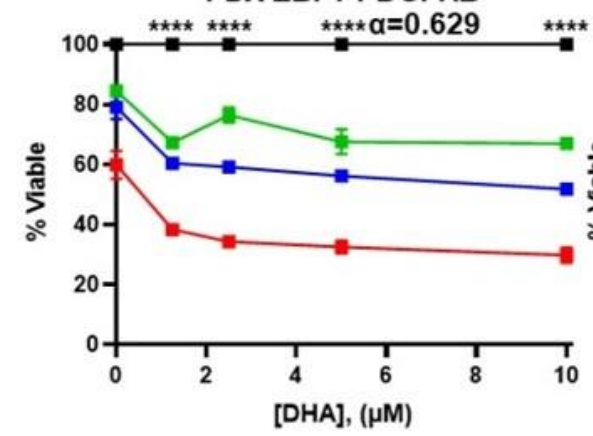

c.

PDX 10940

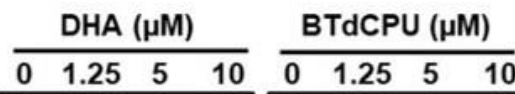

MCL-1 - - - - -

ATF4

Actin
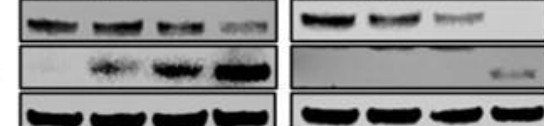

PDX 10940
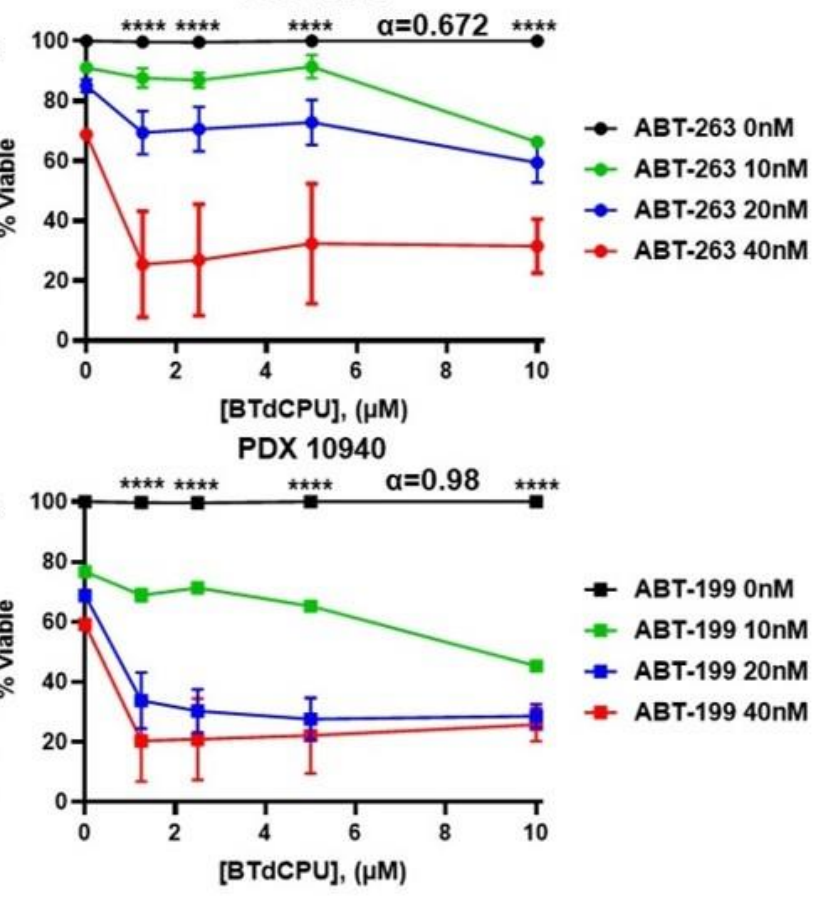

PDX EBF1-PDGFRB

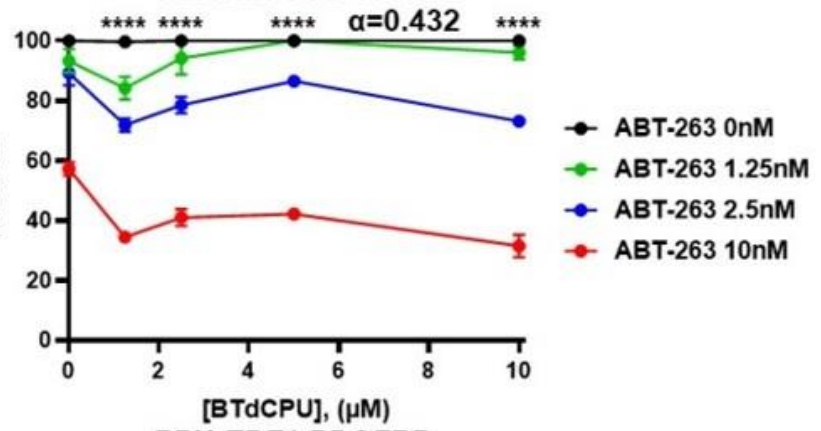

PDX EBF1-PDGFRB

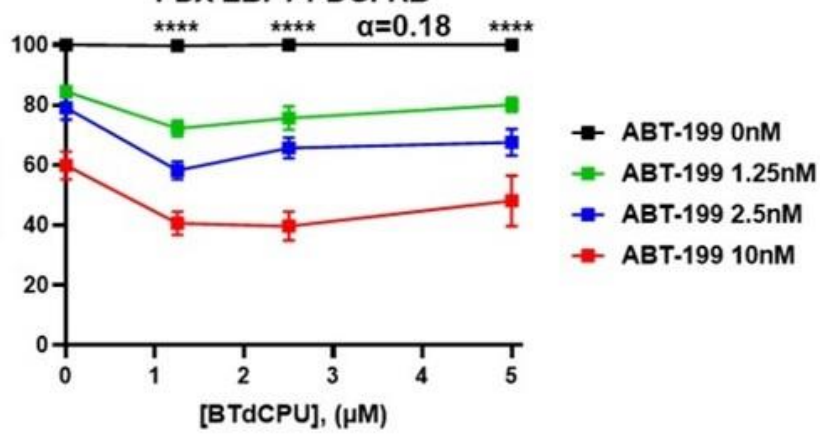

D.

PDX EBF1-PDGFRB

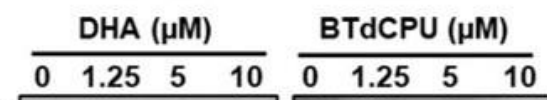

MCL-1 $\sim-\infty$

ATF4

Actin

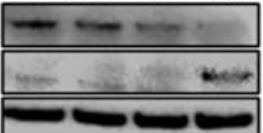



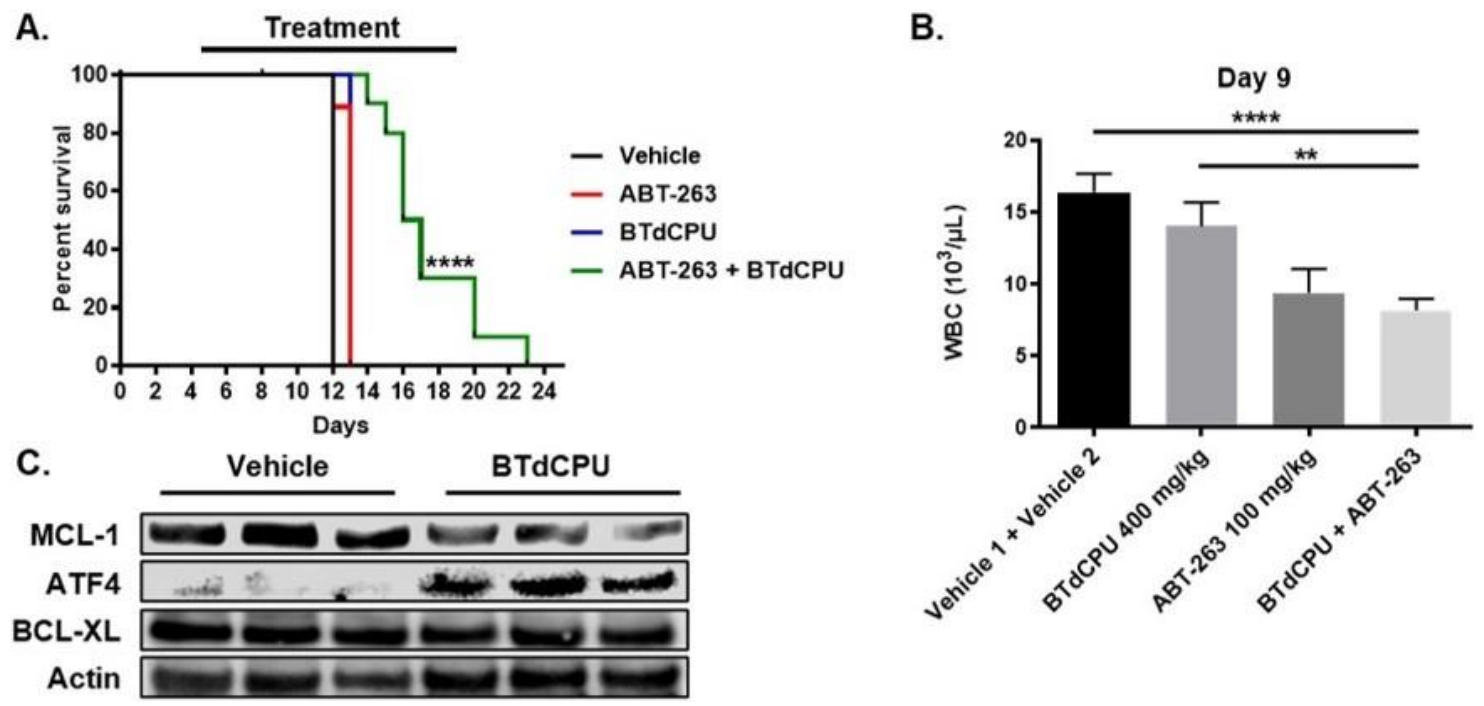

Figure 3-14. HRI activation represses MCL-1 and synergizes with BH3-mimetics in vivo

Mouse BCR-ABL ${ }^{+} \mathrm{B}-\mathrm{ALL}$ cells were injected into unconditioned C57/BL6 recipients. After 5 days, the mice were divided to 4 treatment groups: vehicle, BTdCPU alone (400 $\mathrm{mg} / \mathrm{kg}$ ), ABT-263 alone (100 mg/kg), and combined BTdCPU+ABT-263. Mice were treated daily for 14 days. (A) Kaplan-Meier survival curve the cohorts of mice ( $n=9$ at least for each treatment group). Log-rank test shows $\mathrm{p}<0.0001 * * * *$ for combined treatment group. (B) Total white blood cell (WBC) count in peripheral blood of mice treated with the indicated groups on day 9 after leukemia injection. Each bar represents the average WBC count of the indicated group. Error bars indicate the standard error of mean at least 9 mice per group. Unpaired t-test indicates significance between vehicle and combined treatment group $\mathrm{p}<0.0001 * * * *$. (C) Bone marrow from recipient mice treated with either vehicle or BTdCPU was harvested and immunoblotted for indicated proteins. 
the bone marrow from leukemic mice treated with BTdCPU was significantly decreased when compared to that from mice treated with vehicle alone, whereas BCL-XL expression was unaffected by BTdCPU treatment (Figure 3-14C). Furthermore, cells from mice treated with BTdCPU also exhibited elevated expression of ATF4 indicating the eIF2 $\alpha$ pathway was activated (Figure 3-14C).

Despite 14 days of treatment, the mice from the combination treatment group eventually succumbed to fatal leukemia as determined by immunohistochemical staining for expression of Pax5 and B220 on B-ALL cells (Figure 3-15A). We therefore investigated if the leukemia cells in mice treated with the combination of BTdCPU and ABT-263 had acquired resistance to treatment. To complete this analysis, bone marrow from vehicle or combination treated mice were harvested, expanded, and then re-treated in culture in a synergy assay. Leukemia cells isolated from both mice treated with vehicle and the combination of BTdCPU and ABT-263 still showed a synergistic response to the treatment when treated ex vivo, indicating that the leukemia had not acquired resistance during the in vivo treatment (Figure 3-15B). Taken together, these data indicate that activation of the HRI signal transduction pathway in vivo by BTdCPU can slow leukemia progression when combined with $\mathrm{BH} 3$-mimetic agents by repressing MCL-1 expression. 

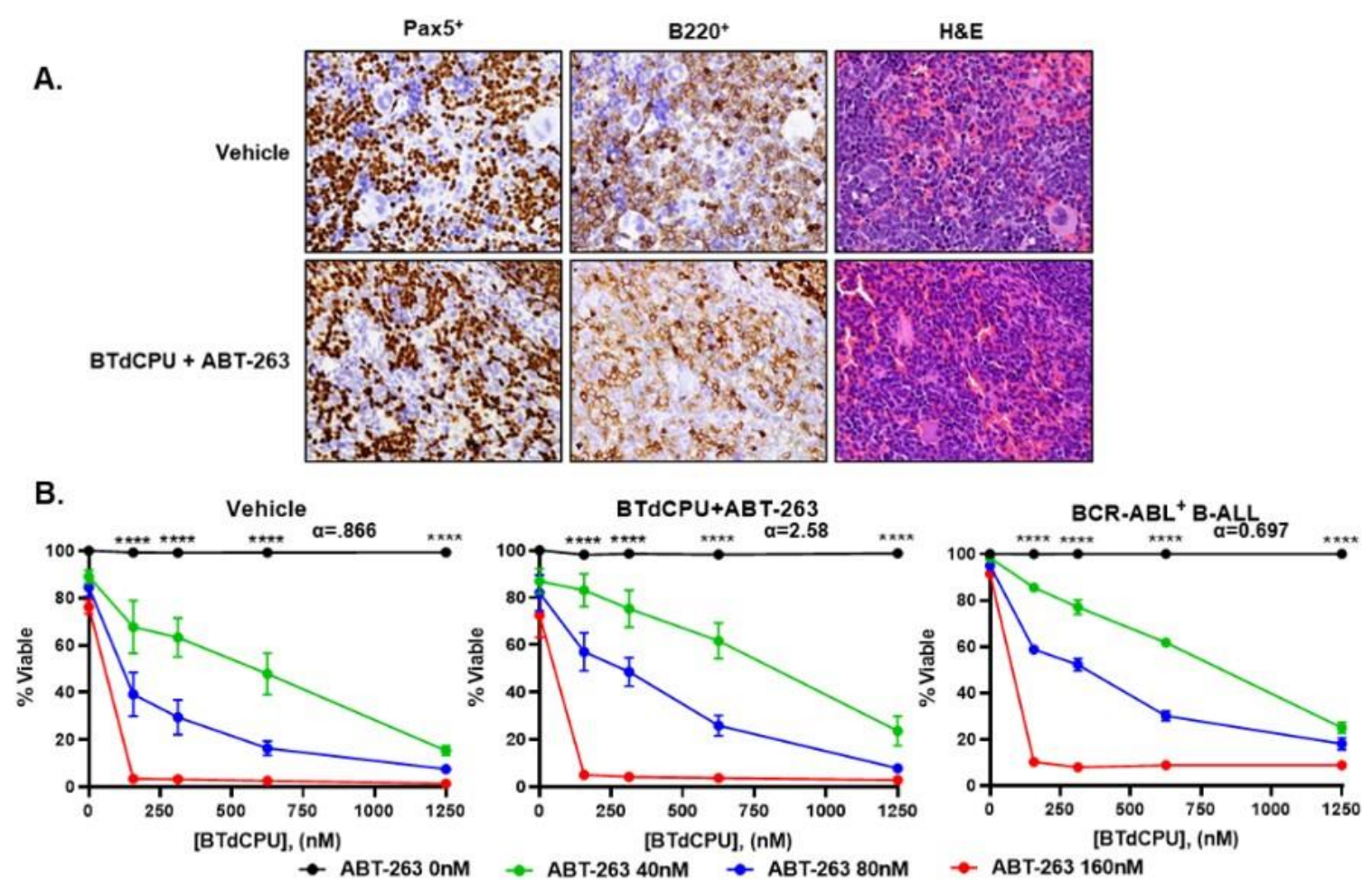

Figure 3-15. Cells do not acquire resistance to HRI activation combined with BH3mimetics in vivo

Mouse BCR-ABL ${ }^{+}$B-ALL cells were injected into unconditioned C57/BL6 recipients. After 5 days, the mice were divided to 4 treatment groups: vehicle, BTdCPU alone (400 $\mathrm{mg} / \mathrm{kg}$ ), ABT-263 alone (100 mg/kg), and combined BTdCPU+ABT-263. Mice were treated daily for 14 days. (A) Immunohistochemistry from spleens of mice bearing BCRABL+ B-ALL treated with either vehicle or BTdCPU + ABT-263 at the time of sacrifice, stained as indicated. (B) Bone marrow harvested from mice treated with either vehicle or the combination of BTdCPU and ABT-263 was harvested and re-cultured with BTdCPU, ABT-263, or a combination of the two. After 24h, viability was measured by Annexin-V and propidium iodide staining. Two-way ANOVA with Bonferroni multiple comparison indicates significance $\mathrm{P}<0.0001 * * * *$ between DHA or BTdCPU alone (0 nM ABT-263) and $160 \mathrm{nM}$ ABT-263 at indicated doses of DHA or BTdCPU. The combination of BTdCPU+ABT-263 showed synergistic response on cells recovered from mice treated with vehicle $(\alpha=0.863, p=3.05 \mathrm{e}-10)$, BTdCPU+ABT-263 $(\alpha=2.58, p=2.36 \mathrm{e}-01)$ and in BCR-ABL ${ }^{+}$B-ALL cells $(\alpha=0.697, p=1.39 \mathrm{e}-13)$. 


\section{CHAPTER 4. DISCUSSION}

\section{Targeting MCL-1 in Cancer}

MCL-1 is among the most frequently amplified genes in human cancer [ $[5]$. This coupled with the role it plays in many other cellular processes, makes MCL-1 an attractive therapeutic target; however, there may be toxicities associated with full inhibition of the protein. MCL-1's structure makes development of specific inhibitors challenging, due to lack of surface features suitable for targeting. Although in recent years there has been progress in the development of MCL-1 inhibitors by structure guided drug discovery, there have also been setbacks. One problem with evaluating the safety and efficacy of these drugs is due to the difference in affinity for mouse and human MCL-1. The first MCL-1 inhibitor to show in vivo activity, S63845, binds to human MCL-1 with an affinity of around 6 times greater than that of mouse MCL-1 [20]. So, although there seem to be no toxicities associated with treating mice with this MCL-1 inhibitor, it doesn't necessarily translate directly to what we may see when humans are treated. One possible way to get by this problem is to evaluate safety in the newly developed humanized MCL1 mouse. This mouse has the mouse Mcll gene replaced with the human MCL1 gene, allowing MCL-1 inhibitors to be evaluated for safety in a way that more accurately represents human treatment with these drugs [99].

A common theory as to how direct MCL-1 inhibitors may be used safely involves the identification of an appropriate "therapeutic window". This is the idea that although genetic deletion of MCL1 may be toxic to many organs, including the heart, inhibiting the protein for more brief periods of time may not have the same detrimental effects. This may be true, and the humanized mouse model may certainly aid in determining what an appropriate level or time of inhibition could be; however, thus far, MCL-1 inhibitor trials have had setbacks. Both Amgen MCL-1 inhibitor clinical trials were placed on hold due to cardiac toxicities, indicating that even though these drugs are inhibiting the protein for therapeutic windows, we still may not be able to avoid cardiac toxicities associated with direct inhibition of MCL-1. These problems stress the importance of finding alternate ways to target this protein.

\section{Dihydroartemisinin}

DHA has been shown previously to be a way to alternatively target MCL-1 [32]. DHA represses MCL-1 protein translation in $\mathrm{BCR}-\mathrm{ABL}^{+} \mathrm{B}-\mathrm{ALL}$, a cancer known to depend on MCL-1 for survival. Our data now reveal that this is through an HRIdependent induction of the integrated stress response. We show that DHA disrupts the interaction between heme and HRI, which allows HRI to become an active kinase, phosphorylate eIF2 $\alpha$, and ultimately shut down global cap-dependent protein translation. Due to the rapid turnover properties of MCL-1, this allows for a repression of MCL-1, and not the other BCL-2 family members. It is possible that other short-lived proteins in 
the cell may be affected similarly to MCL-1, and this remains an open question for future studies.

The repression of MCL-1 through the mechanism of HRI activation (by DHA or BTdCPU) appears to be well tolerated. DHA combined with ABT-263 was previously shown to be well tolerated and effective in the treatment of mice bearing $\mathrm{BCR}-\mathrm{ABL}^{+} \mathrm{B}-$ ALL [32], and here we show that BTdCPU combined with ABT-263 gives a similar result. This mechanism of MCL-1 repression, rather than direct inhibition of the BH3binding pocket, may represent a safer way to target the protein. This has major implications in the field of cancer biology; MCL-1 plays a role in so many types of cancer and the direct inhibition appears to be too toxic to tolerate in patients. The repression of the protein by DHA may be a safe way to target the protein, while still allowing enough expression to fulfill its functions in the body aside from preventing apoptosis in cancer cells.

\section{Cellular Stress Response Pathway}

\section{Heme Sensing Pathway}

This study represents the first time the cellular heme pathway has been linked to MCL-1. By genome-wide CRISPR screening, we show that heme synthesis is required for the induction of apoptosis by DHA. This led us to find that DHA acts to repress MCL-1 by disrupting the interaction between heme and HRI, thus activating HRI. We further show that the activation of HRI, by DHA and the direct activator BTdCPU, can lead to an MCL-1 translational repression.

Heme Levels in Cancer Cells. In addition to the possibility of targeting MCL-1 by the HRI heme-sensing pathway being safer, it may also be more selective to cancer cells compared to other cells. It is known that some cancer cells have increased heme synthesis and expression of genes involved in the heme synthesis pathway, [85] including both solid tumors and hematological malignancies $[\underline{85}, \underline{100]}$. One example is seen in pediatric AML cells where it was shown that these patients exhibit increased expression of genes involved in heme biosynthesis, including ALAD, HMBS, UROS, UROD, CPOX, PPOX, and FECH [85]. Additionally, UROD expression predicted poor overall survival and earlier death among adult AML patients. Although we have not yet tested the efficacy of DHA in killing AML cells, this study would suggest that DHA could be efficacious and more selective to AML cells, because they have higher expression of heme synthesis genes.

An example of solid tumor cells having increased heme synthesis can be seen in lung cancer [100]. It has been shown that non-small cell lung cancer cells have increased heme synthesis and elevated ALAS1 expression compared to normal lung epithelial cells [100]. Based on our data showing that DHA more potently targets cells with higher amounts of heme synthesis, one could imagine that DHA may be selective for these cells, 
or any type of cancer cells that have higher heme levels, compared to non-cancerous cells. This again speaks to the potential safety of DHA; it may be more toxic to cancer cells due to their higher heme levels, and; therefore, safer to use because it may not affect normal cells as potently.

Targeting the Heme Sensing Pathway. In addition to DHA, there could other ways to target this heme sensing pathway. Although BTdCPU is a small molecule activator of HRI, it is not necessarily a drug, it provides evidence that the activation of HRI by other compounds leads to the same result of MCL-1 repression. There is interest in generating drugs which activate HRI specifically, and there have been recent advances in the development of HRI activators with improved biophysical properties, based on cLogPs [101]. Though these new drugs were shown to phosphorylate eIF2 $\alpha$ and induce CHOP in melanoma cells, there is currently no in vivo data available. The authors of this drug discovery study argue that HRI activators would be useful in the context of hemoglobin disorders, such as $\beta$-thalassemia. Our data provides evidence for the use of these in the context of $\mathrm{Ph}^{+}$and $\mathrm{Ph}$-like B-ALL, and potentially any other cancers dependent on MCL-1. Furthermore, our data highlights the importance of further development of HRI activators because of the possibility of such a wide range of use. MCL-1 has been shown to be important in such a broad range of cancers so one can imagine the impact of the development of a specific activator of HRI that could be used in the clinic. Additionally, we show that the combination of HRI-mediated MCL-1 repression synergizes with BH3-mimetics, including FDA approved ABT-199. This again highlights the impact of this discovery that HRI activation can lead to synergy with approved drugs, by implying how useful an HRI specific activator could be.

Another way HRI could possibly be targeted is through decreasing heme levels, such as with SA. We show that the treatment with SA does lead to an MCL-1 repression, though it does take longer than repression by DHA (Figure 3-5D). In fact, we are not the first to show a relationship between SA and the BCL-2 family. Lin, et al [102] showed through a CRISPR screen that the loss of heme synthesis sensitizes cells to venetoclax. They went on to show that SA combined with venetoclax kills AML cells in a synergistic manner. Although they speculated that this effect was due to mitochondrial defects and electron transport chain dysfunction, they did not investigate the role of MCL-1 in this process. We would argue that the sensitization to venetoclax by SA is due to a reduction of heme, which activates HRI and represses MCL-1, thus sensitizing the cells to the BCL-2 inhibition. The combination of results from our studies could be impactful in multiple ways. First, if it is true that SA sensitizes cells to other drugs by repressing MCL-1, it highlights a way we can target MCL-1 (through heme synthesis inhibition). Second, these studies suggest that the combination of HRI activation and BH3-mimetics is synergistic in AML cells. This is important, as it adds to the potential types of cancer this pathway could be exploited in. 


\section{Targeting Other Kinases}

In addition to HRI, there are three other kinases of eIF2 $\alpha$. We would speculate that activation of any of the other three would lead to a similar MCL-1 repression and synergy with BH3-mimetics. We, and others, have shown that thapsigargin, an activator of PERK through ER stress, induces MCL-1 repression (Figure 3-8B) [103].

Thapsigargin is an investigational tool, not an actual drug, but it does support the thought that other methods of ISR activation could repress MCL-1. There are other drugs being developed to target this pathway. One recent study tested MK-28, which is a specific PERK activator, suggested to interact with the PERK activation loop, and was shown to have favorable pharmacokinetics and penetrate the mouse blood brain barrier [104]. Again, this drug was not generated with the idea of using it to repress MCL-1 but we would argue that MCL-1 repression could be induced by it.

Other PERK activators have been shown to induce apoptosis in cancer cells. CCT020312, a selective PERK activator, was shown to induce eIF2 $\alpha$ phosphorylation, induction of and ATF4 and CHOP, and induced apoptosis in a human breast cancer cell line [105]. This study also suggested the PERK activator led to an induction of BAX and a repression of BCL-2, though they did not speculate on a mechanism, and they showed no data on MCL-1 expression. The results of our work highlight the importance of further investigation and development of these agents for potential use as drugs to target MCL-1 through alternative mechanisms.

\section{Potential for Combinatorial Uses}

Another possible impact of our work is the potential for DHA to be used in combination with other therapeutics. DHA is an already approved anti-malarial drug used around the world; therefore, one can imagine it would be safe to use for treatment of other diseases. We have demonstrated the synergistic effect of combining DHA with BH3-mimetics, this is important because it could reduce toxicities associated with these drugs, and it could prevent MCL-1 from generating resistance to these drugs. We would also speculate that DHA could be used in combination with other approved drugs which MCL-1 is known to cause resistance in.

\section{BH3-Mimetics}

Venetoclax is an FDA approved BCL-2 inhibitor for which MCL-1 is a known resistance factor [106]. Our data shows that the combination of DHA synergizes with venetoclax due to the repression of MCL-1. Treating patients with this combination may avoid the development of resistant disease because MCL-1 will be unable to compensate for the BCL-2 inhibition. When cells are exposed to venetoclax the upregulation of MCL-1 is believed to generate resistance [107]; by combining the inhibition of MCL-1 by DHA with venetoclax the potential for MCL-1 upregulation should be prevented and the development of resistance should be less-likely. 
Navitoclax is an inhibitor of BCL-2, BCL-XL, and BCL-W, and although it is potent in killing cancer cells, it has associated toxicities. On-target inhibition of BCL-XL leads to thrombocytopenia; thus, limiting the clinical viability of navitoclax. Our data strongly supports the idea that navitoclax would be potent in the clinic when combined with DHA. This synergistic effect may be useful because it allows navitoclax to be used at a much lower concentration than what would be effective as a single agent, which may be low enough to avoid causing toxicities. This could be incredibly impactful because much work has been done on the development and pre-clinical studies of effectiveness across many types of cancer, and by combining DHA with navitoclax it may allow this drug to safely reach the clinic and effectively treat patients.

\section{Approved Therapeutics}

Again, MCL-1 is a known resistance factor of many cancer therapeutics. This highlights potential uses of DHA in combination with many drugs. It has been shown that silencing MCL1 (by siRNA) can sensitize various types of cancer cells to approved therapeutics including vemurafenib, imatinib, gefitinib, crizotinib, and lapatinib, and that treatment with these drugs induces a dependence on MCL-1 [11]. The combination of DHA with these drugs may be beneficial because, again, the DHA would repress MCL-1 and prevent development of resistance due to the increased dependence on its expression. It is also important to note that these drugs are approved; so you would imagine that the combination of DHA with any of these should be safe, as each are safe as single agents, though this would need to be evaluated for confirmation.

\section{Therapeutic Impact}

We show improved efficacious treatment of both $\mathrm{Ph}^{+}$and $\mathrm{Ph}$-like $\mathrm{B}-\mathrm{ALL}$ with DHA combined with BH3 mimetics. Both subtypes need new therapeutic options, due to emergent resistance and treatment challenges. Though tyrosine kinase inhibitors are a promising treatment option for $\mathrm{Ph}^{+} \mathrm{ALL}$, resistance does emerge; and Ph-like ALL has the additional challenge of understanding what kinase to target. Our data demonstrate that DHA could be a valid option for both poor-prognosis leukemia subtypes. The treatment of these cancers with the combination of DHA and $\mathrm{BH} 3$-mimetics should have a major impact on the ALL field. DHA and BH3-mimetics would be a broader treatment option which would potentially prevent the emergence of resistance. The broad treatment by DHA and BH3-mimetics may also be more applicable to the Ph-like subtype because it does not come with the burden of figuring out what to target in these cells. We have shown this combination is effective against cells with the Pax5-Jak2, RCSD1-ABL1, and $R S C D 1-A B L 2$ fusions, as well as PDX cells with the EBF1-PDGFRB fusion. This is important as it highlights the notion that this treatment is effective in Ph-like ALL cells harboring a variety of the mutations seen in this disease. 


\section{CHAPTER 5. FUTURE DIRECTIONS}

\section{Spectrum of Cancers Sensitive to DHA}

One aspect of this project that remains to be investigated is the diverse range of cancers in which this treatment could be effective. Based on the amount of cancers that are known to be dependent on MCL-1, either for initiation or survival, one could imagine DHA being an effective treatment strategy in many cancers; however, this is yet to be confirmed. Additionally, it would be interesting to note if this would be effective in both solid tumors and hematological malignancies, or if it is limited to one tumor type.

\section{AML}

AML is a disease in which the survival rate is much poorer than that of B-ALL. The BCL-2 family has long been associated with AML and is known to be involved in disease pathogenesis and resistance to therapeutics. Studies have shown that the combination of MCL-1 inhibition with BH3-mimetics is effective in killing AML cells [22] so it is easy to imagine that DHA combined with venetoclax would be an effective treatment. Additionally, it has been shown that the combination of heme synthesis inhibition with venetoclax is toxic to AML cells [102]. Though this study did not investigate the role of MCL-1 expression, we would argue that the heme synthesis inhibition would repress MCL-1, and the combination of DHA with BH3-mimetics would give the same result. We do have evidence of DHA leading to an MCL-1 repression and ATF4 induction in a panel of human AML cell lines, indicating that the results presented in B-ALL cells hold true in this cancer as well; however, the synergy of DHA and BH3-mimetics remains to be experimentally evaluated (Figure 5-1).

\section{Multiple Myeloma}

Multiple myeloma is another disease in which it would be interesting to evaluate the effects of DHA treatment. The response rate for newly diagnosed patients to frontline treatment, dexamethasone, is roughly $42 \%$, highlighting the importance of finding new therapeutics for this disease [108]. It is known that some multiple myeloma cell lines, H929 cells for example, are dependent on MCL-1 for survival. This would indicate that repression of MCL-1 by DHA could be an effective treatment. Additionally, it has been shown that dexamethasone resistant multiple myeloma cells are sensitive to BTdCPU [108]. Again, this study did not comment on MCL-1 expression, but we would argue this sensitivity could be due to MCL-1 repression. BTdCPU is not an approved drug but it would be interesting to see if DHA provided the same sensitivity, and if the combination of DHA with BH3-mimetics would be effective in this type of cancer. 


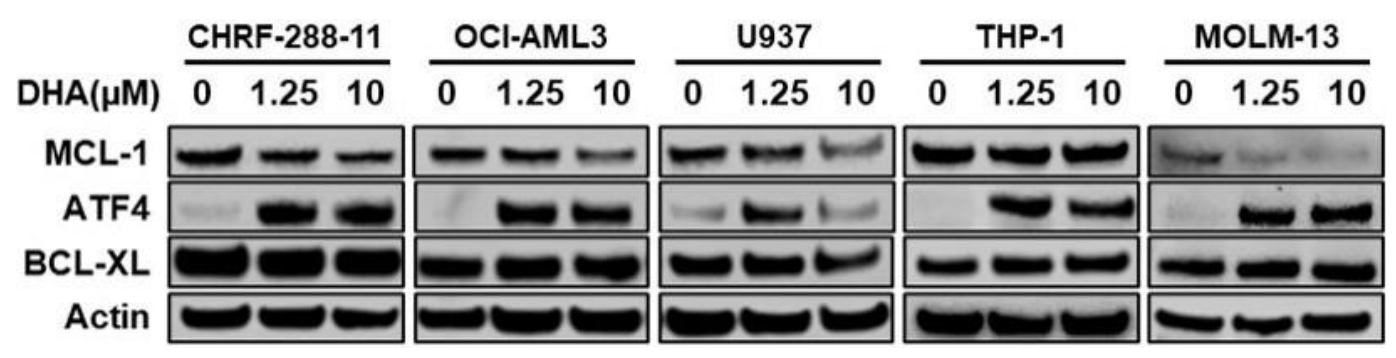

Figure 5-1. DHA represses MCL-1 in a panel of human AML cell lines Human AML cell lines (CHRF-288-11, OCI-AML3, U937, THP-1, MOLM-13) were treated with $1.25 \mu \mathrm{M}$ or $10 \mu \mathrm{M}$ DHA for $24 \mathrm{~h}$ and protein expression was determined by immunoblotting with indicated antibodies. 


\section{Breast Cancer}

A third cancer that would be interesting to evaluate the effects of DHA in would be breast cancer. It has been shown that MCL-1 plays a role in the development of chemotherapy resistance in breast cancer stem cells [109], so it would be easy to imagine that DHA could be effective in treating breast cancer. Additionally, MCL-1 inhibitors have been shown to be effective in some breast cancer cells as a single agent or in combination with other available therapies $[20,110]$. It would also be interesting to see if MCL-1 is repressed in solid tumor cells in vivo by DHA, or other HRI activators. We show that MCL-1 is repressed in bone marrow taken from leukemia bearing mice treated with BTdCPU, but it has not been examined if solid tumor cells taken from mice treated with DHA or BTdCPU would have a repression of MCL-1. If this were found to be true, it would broaden the scope of potential uses of DHA dramatically.

\section{The Separation of ISR Activation and Apoptosis}

Our genome-wide CRISPR screen revealed that DHA requires both heme synthesis and intrinsic apoptosis to kill BCR-ABL ${ }^{+} \mathrm{B}-\mathrm{ALL}$ cells. We have thoroughly investigated the connection between heme and the HRI pathway leading to MCL-1 repression; however, we still do not know what role certain genes of the apoptotic pathway are playing. DHA represses MCL-1 at much lower concentrations than what it needs to induce apoptosis, indicating that the way DHA influences the two pathways may be different. We also know that both NOXA and PUMA are induced by DHA, but neither were a hit in the CRISPR screen, indicating that as a single gene knockout they may not be required for DHA to induce apoptosis.

As an anti-malarial, DHA acts by inducing ROS; one could hypothesize that to kill cancer cells, DHA generates ROS, which leads to DNA damage, activation of p53 and induction of its downstream targets NOXA and PUMA. We have shown that ROS induction plays no role in the induction of apoptosis in these cells or the repression of MCL-1 (Figure 5-2A, B) [32]. DHA does induce ROS, which can be scavenged using the superoxide dismutase mimetic, MnTMPyP; however, this provides no protection to DHA induced apoptosis (Figure 5-2A, B). Additionally, in the presence of ROS inhibitors, we still see p53 and PUMA induction, as well as $\gamma \mathrm{H} 2 \mathrm{AX}$, which is indicative of DNA double stranded breaks (Figure 5-2C, D). Therefore, it is possible that DHA is inducing DNA damage independent of ROS induction. This DNA damage could be responsible for a p53 and subsequent NOXA and PUMA induction. Investigating this mechanism of DNA damage could provide more insight into how DHA kills cells as a single agent, as compared to how it works synergistically through the repression of MCL1 .

In addition to the hypothesis that DNA damage is responsible for the p53 induction, there is also a possibility that p53 is being activated through a pathway involving heme. A recent publication shows that p53 can be activated by a decrease in heme [111]. The authors claim that excess cellular iron levels lead to increased heme 
A.

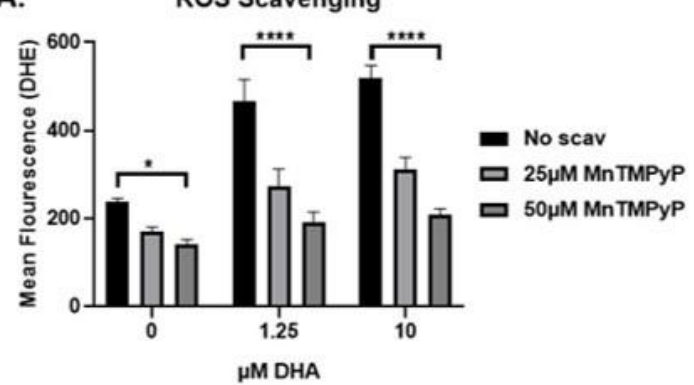

B.

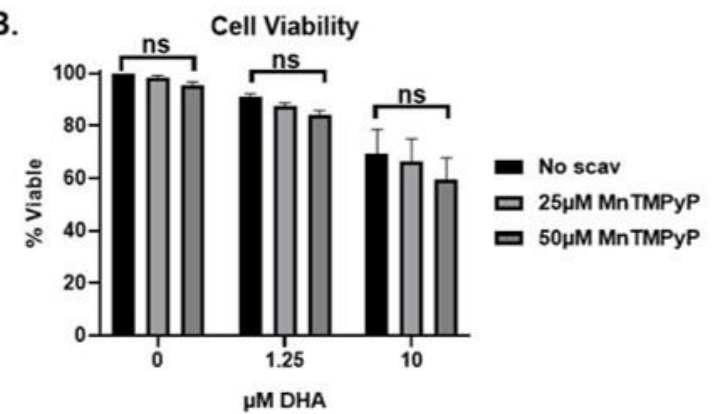

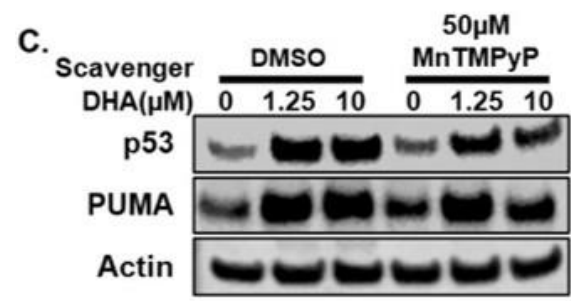

D.
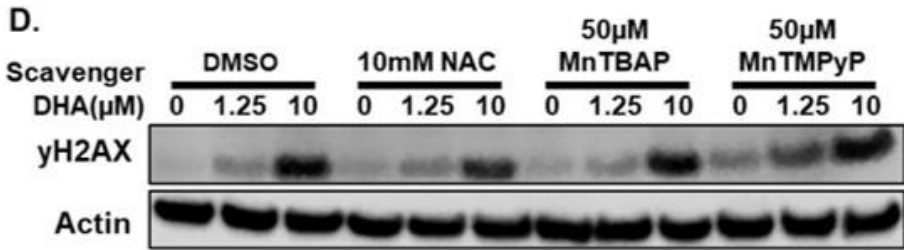

Figure 5-2. DHA-induced apoptosis is ROS independent

$\mathrm{BCR}-\mathrm{ABL}^{+}$cells were pre-treated with the indicated doses of ROS scavengers

(MnTMPyP, NAC, MnTBAP) for 2h, DHA was then added to the cells for 16h. (A) ROS was measured by staining with DHE and analyzing by flow cytometry. Two-way ANOVA with Bonferroni multiple comparison indicates significance $\mathrm{p}<0.05^{*}$, $\mathrm{p}<0.0001 * * * *$ between 50 $\mu \mathrm{M}$ MnTMPYP and no scavenger controls. (B) Viability was measured by Annexin-V and propidium iodide staining. Two-way ANOVA with Bonferroni multiple comparison indicates no significant differences (ns). (C-D) Protein expression was determined by immunoblotting with indicated antibodies. 
levels; this heme can then bind p53 and trigger its nuclear export, through CRM1, and ubiquitin mediated degradation. Conversely, during iron deprivation, p53 is not bound to heme or degraded; therefore, it is upregulated and its tumor suppressor function is increased. If this heme dependent regulation of p53 is true, then it is possible that DHA binding to heme prevents the heme-mediated degradation of $\mathrm{p} 53$, allowing it to be stabilized and active. To test this hypothesis, a similar experiment to that of the heme and HRI spectroscopy experiment could be performed. If heme is generally leading to the degradation of p53 by direct binding, when we add heme and p53 together we would expect to see a Soret peak formed. Then, if DHA is disrupting the binding which is allowing p53 to be stabilized and active when we add DHA to the p53 and heme we should see a loss of the Soret peak. This would indicate that DHA is inducing p53 activity through prevention of its binding to heme and subsequent degradation.

Another interesting aspect of signaling downstream of DHA treatment is the induction of NOXA and PUMA. Although p53 can induce NOXA and PUMA, they can also both be induced because of ISR induction. NOXA and PUMA are both proapoptotic proteins which are known to be able to bind to MCL-1. Because BCR-ABL ${ }^{+}$ B-ALL cells are known to be dependent on MCL-1 for survival it is reasonable to hypothesize that as a single agent, DHA induces the ISR, which leads to an induction of NOXA and PUMA, allowing them to increase binding with MCL-1, ultimately leading to cell death. Though we have not tested this hypothesis, it would be interesting to see if DHA treatment increased the binding of NOXA and PUMA to MCL-1 by immunoprecipitation. Furthermore, repeating the experiment on Eif2akl KO cells would shed light on the hypothesis that the ISR induction could be responsible for the NOXA and PUMA induction.

\section{Potential Effects of DHA}

We have focused most on the mechanism by which DHA acts to repress MCL-1; however, there is the potential for other effects of DHA that we have not uncovered. We have shown that HRI activation represses MCL-1 translation. This effect is made possible by the short-lived nature of the MCL-1 protein. It is rational to imagine that any other short-lived protein may be affected like MCL-1. We have shown that the other anti-apoptotic members of the BCL-2 family are unaffected; however, it is known that they have a much longer half-life. It would be interesting to investigate if any other short-lived proteins are repressed, and based on their function, possibly try to exploit their repression as a therapeutic.

Another potential effect of using HRI activation, by DHA or other drugs, as a cancer therapeutic is the possibility that it will affect non-cancerous cells. Both DHA and BTdCPU in combination with ABT-263 extended survival in leukemia bearing mice without any overt toxicities. This is encouraging; however, we did not look at ISR activation in non-cancerous cells. It is possible, that DHA more selectively targets cancer cells due to higher heme levels, however, we did not fully investigate this possibility. It would be interesting to note if $\mathrm{CHOP}$ and ATF4 are induced in normal hematopoietic 
cells in response to DHA and determine if MCL-1 is repressed in those cells. Even if MCL-1 is repressed in normal cells, we would predict that it would still not be as toxic as a direct inhibitor of MCL-1. We saw no evidence of toxicities in mice treated with DHA or BTdCPU including that of cardiac failure, but it would still be important to note if there is ISR induction in non-cancerous cells after DHA treatment.

\section{DHA and Heme Interaction}

Another open question relates to how exactly DHA interacts with heme to disrupt the heme and HRI interaction. We showed by spectroscopy on purified proteins that DHA disrupts the interaction between heme and HRI; however, we did not investigate how this is taking place. Based on its mechanism as an anti-malarial, it is possible that DHA binds to the iron in heme which may block the heme and HRI interaction site. One can imagine that to test this hypothesis, an iron chelator could be used to sequester cellular iron, and if this prevents any induction of ISR activation by DHA, you could conclude that DHA must interact with the iron from heme to block the HRI and heme interaction. Determining how exactly DHA blocks the interaction of heme with HRI could be useful in drug development. Rather than trying to generate a drug that directly binds to and activates HRI, a drug could be designed to bind heme and/or iron to prevent the HRI-heme interaction, thus activating HRI. It is unclear as to if this would be safer or more effective than direct HRI activation, but it could be worth exploring.

\section{Investigation of Potential Drug Combinations}

An interesting aspect of DHA, or HRI activation by other mechanisms, that we have not yet explored is the potential number of drugs with which it could synergize. Thus far, we have only looked at synergy between DHA and BH3-mimetics, but we would predict that these are not the only class of drugs with which DHA may synergize.

It would be interesting to see if DHA could synergize with any frontline treatments which are already approved and currently used in the clinic. Due to the dependence of $\mathrm{Ph}^{+} \mathrm{B}-$ ALL on MCL-1 and the development of resistance to TKIs, the combination of HRI activation and TKIs could be an effective therapeutic option. It has previously been shown that the combination of DHA and dasatinib is effective in treating mice bearing BCR-ABL leukemia; including BCR-ABL ${ }^{\mathrm{T} 315 \mathrm{I}}$ which is resistant to dasatinib as a single agent [40]. This supports the idea that HRI activation could be effective in combination with FDA approved TKIs. Another potential therapeutic combination could be DHA and prednisolone. Silencing MCL-1 has been shown to play a role in sensitizing ALL cells to prednisolone, therefore it would be reasonable to hypothesize that repressing MCL-1 by DHA would give the same sensitization [112]. Due to the role of MCL-1 in drug resistance it would be reasonable to evaluate the synergy between DHA and any drug in which MCL-1 is known to confer resistance to. This would be an interesting avenue to explore, because if synergy is found between DHA and any other approved agents you would imagine that this combinatorial treatment would be able to move rapidly to the clinic due to both agents being approved drugs. Also, if it is found that DHA synergizes 
with many agents it could be used as a standard chemotherapeutic in a wide array of cancer types. 


\section{LIST OF REFERENCES}

1. Youle, R.J. and A. Strasser, The BCL-2 protein family: opposing activities that mediate cell death. Nat Rev Mol Cell Biol, 2008. 9(1): p. 47-59. https://doi.org/10.1038/nrm2308.

2. Pihan, P., A. Carreras-Sureda, and C. Hetz, BCL-2 family: integrating stress responses at the ER to control cell demise. Cell Death Differ, 2017. 24(9): p. 1478-1487. https://doi.org/10.1038/cdd.2017.82.

3. Certo, M., et al., Mitochondria primed by death signals determine cellular addiction to antiapoptotic BCL-2 family members. Cancer Cell, 2006. 9(5): p. 351-65. https://doi.org/10.1016/j.ccr.2006.03.027.

4. Li, K., Interdiction at a protein-protein interface: MCL-1 inhibitors for oncology. Bioorg Med Chem Lett, 2021. 32: p. 127717. https://doi.org/10.1016/j.bmcl.2020.127717.

5. Beroukhim, R., et al., The landscape of somatic copy-number alteration across human cancers. Nature, 2010. 463(7283): p. 899-905. https://doi.org/10.1038/nature08822.

6. Hanahan, D. and R.A. Weinberg, Hallmarks of cancer: the next generation. Cell, 2011. 144(5): p. 646-74. https://doi.org/10.1016/j.cell.2011.02.013.

7. Vaux, D.L., S. Cory, and J.M. Adams, Bcl-2 gene promotes haemopoietic cell survival and cooperates with c-myc to immortalize pre-B cells. Nature, 1988. 335(6189): p. 440-2. https://doi.org/10.1038/335440a0.

8. Delbridge, A.R., et al., Thirty years of BCL-2: translating cell death discoveries into novel cancer therapies. Nat Rev Cancer, 2016. 16(2): p. 99-109. https://doi.org/10.1038/nrc.2015.17.

9. Weiss, L.M., et al., Molecular analysis of the 1 (14;18) chromosomal translocation in malignant lymphomas. N Engl J Med, 1987. 317(19): p. 1185-9.

https://doi.org/10.1056/NEJM198711053171904.

10. Wendel, H.G., et al., Dissecting eIF4E action in tumorigenesis. Genes Dev, 2007. 21(24): p. 3232-7. https://doi.org/10.1101/gad.1604407.

11. Montero, J., et al., Destabilization of NOXA mRNA as a common resistance mechanism to targeted therapies. Nat Commun, 2019. 10(1): p. 5157. https://doi.org/10.1038/s41467-019-12477-y.

12. Lestini, B.J., et al., Mcl1 downregulation sensitizes neuroblastoma to cytotoxic chemotherapy and small molecule Bcl2-family antagonists. Cancer Biol Ther, 2009. 8(16): p. 1587-95. https://doi.org/10.4161/cbt.8.16.8964.

13. Osaki, S., et al., Ablation of MCL1 expression by virally induced microRNA-29 reverses chemoresistance in human osteosarcomas. Sci Rep, 2016. 6: p. 28953. https://doi.org/10.1038/srep28953.

14. Tahir, S.K., et al., Potential mechanisms of resistance to venetoclax and strategies to circumvent it. BMC Cancer, 2017. 17(1): p. 399.

https://doi.org/10.1186/s12885-017-3383-5.

15. Oltersdorf, T., et al., An inhibitor of Bcl-2 family proteins induces regression of solid tumours. Nature, 2005. 435(7042): p. 677-81. https://doi.org/10.1038/nature03579. 
16. Suvarna, V., V. Singh, and M. Murahari, Current overview on the clinical update of Bcl-2 anti-apoptotic inhibitors for cancer therapy. Eur J Pharmacol, 2019. 862: p. 172655. https://doi.org/10.1016/j.ejphar.2019.172655.

17. Wilson, W.H., et al., Navitoclax, a targeted high-affinity inhibitor of BCL-2, in lymphoid malignancies: a phase 1 dose-escalation study of safety, pharmacokinetics, pharmacodynamics, and antitumour activity. Lancet Oncol, 2010. 11(12): p. 1149-59. https://doi.org/10.1016/S1470-2045(10)70261-8.

18. Drugs.com. Venclexta FDA Approval History. 2021 [cited 2021.

19. Hird, A.W. and A.E. Tron, Recent advances in the development of Mcl-1 inhibitors for cancer therapy. Pharmacol Ther, 2019. 198: p. 59-67. https://doi.org/10.1016/j.pharmthera.2019.02.007.

20. Kotschy, A., et al., The MCL1 inhibitor S63845 is tolerable and effective in diverse cancer models. Nature, 2016. 538(7626): p. 477-482. https://doi.org/10.1038/nature19830.

21. Yi, X., et al., AMG-176, an Mcl-1 Antagonist, Shows Preclinical Efficacy in Chronic Lymphocytic Leukemia. Clin Cancer Res, 2020. 26(14): p. 3856-3867. https://doi.org/10.1158/1078-0432.CCR-19-1397.

22. Tron, A.E., et al., Discovery of Mcl-1-specific inhibitor AZD5991 and preclinical activity in multiple myeloma and acute myeloid leukemia. Nat Commun, 2018. 9(1): p. 5341. https://doi.org/10.1038/s41467-018-07551-w.

23. Wang, X., et al., Deletion of MCL-1 causes lethal cardiac failure and mitochondrial dysfunction. Genes Dev, 2013. 27(12): p. 1351-64. https://doi.org/10.1101/gad.215855.113.

24. Thomas, R.L., et al., Loss of MCL-1 leads to impaired autophagy and rapid development of heart failure. Genes Dev, 2013. 27(12): p. 1365-77. https://doi.org/10.1101/gad.215871.113.

25. Opferman, J.T., et al., Obligate role of anti-apoptotic MCL-1 in the survival of hematopoietic stem cells. Science, 2005. 307(5712): p. 1101-4. https://doi.org/10.1126/science.1106114.

26. Opferman, J.T., et al., Development and maintenance of B and T lymphocytes requires antiapoptotic MCL-1. Nature, 2003. 426(6967): p. 671-6. https://doi.org/10.1038/nature02067.

27. Vikstrom, I., et al., Mcl-1 is essential for germinal center formation and B cell memory. Science, 2010. 330(6007): p. 1095-9. https://doi.org/10.1126/science.1191793.

28. Huntington, N.D., et al., Interleukin 15-mediated survival of natural killer cells is determined by interactions among Bim, Noxa and Mcl-1. Nat Immunol, 2007. 8(8): p. 856-63. https://doi.org/10.1038/ni1487.

29. Vick, B., et al., Knockout of myeloid cell leukemia-1 induces liver damage and increases apoptosis susceptibility of murine hepatocytes. Hepatology, 2009. 49(2): p. 627-36. https://doi.org/10.1002/hep.22664.

30. Rooswinkel, R.W., et al., Antiapoptotic potency of Bcl-2 proteins primarily relies on their stability, not binding selectivity. Blood, 2014. 123(18): p. 2806-15. https://doi.org/10.1182/blood-2013-08-519470. 
31. Cidado, J., et al., AZD4573 Is a Highly Selective CDK9 Inhibitor That Suppresses MCL-1 and Induces Apoptosis in Hematologic Cancer Cells. Clin Cancer Res, 2020. 26(4): p. 922-934. https://doi.org/10.1158/1078-0432.CCR-19-1853.

32. Budhraja, A., et al., Modulation of Navitoclax Sensitivity by DihydroartemisininMediated MCL-1 Repression in BCR-ABL(+) B-Lineage Acute Lymphoblastic Leukemia. Clin Cancer Res, 2017. 23(24): p. 7558-7568. https://doi.org/10.1158/1078-0432.CCR-17-1231.

33. Gutman, J., et al., Safety, tolerability, and efficacy of repeated doses of dihydroartemisinin-piperaquine for prevention and treatment of malaria: a systematic review and meta-analysis. Lancet Infect Dis, 2017. 17(2): p. 184-193. https://doi.org/10.1016/S1473-3099(16)30378-4.

34. Meshnick, S.R., Artemisinin: mechanisms of action, resistance and toxicity. Int J Parasitol, 2002. 32(13): p. 1655-60. https://doi.org/10.1016/s00207519(02)00194-7.

35. Bottineau, M.-C., et al., Essential drugs - practical guidelines. DIHYDROARTEMISININ/PIPERAQUINE = DHA/PPQ oral. Médecins Sans Frontières.

36. Meshnick, S.R., et al., Artemisinin (qinghaosu): the role of intracellular hemin in its mechanism of antimalarial action. Mol Biochem Parasitol, 1991. 49(2): p. 181-9. https://doi.org/10.1016/0166-6851(91)90062-b.

37. Hong, Y.L., Y.Z. Yang, and S.R. Meshnick, The interaction of artemisinin with malarial hemozoin. Mol Biochem Parasitol, 1994. 63(1): p. 121-8. https://doi.org/10.1016/0166-6851(94)90014-0.

38. Yang, Y.Z., B. Little, and S.R. Meshnick, Alkylation of proteins by artemisinin. Effects of heme, pH, and drug structure. Biochem Pharmacol, 1994. 48(3): p. 56973. https://doi.org/10.1016/0006-2952(94)90287-9.

39. Slezakova, S. and J. Ruda-Kucerova, Anticancer Activity of Artemisinin and its Derivatives. Anticancer Res, 2017. 37(11): p. 5995-6003. https://doi.org/10.21873/anticanres.12046.

40. Singh, H., et al., A screening-based approach to circumvent tumor microenvironment-driven intrinsic resistance to BCR-ABL+ inhibitors in Ph+ acute lymphoblastic leukemia. J Biomol Screen, 2014. 19(1): p. 158-67. https://doi.org/10.1177/1087057113501081.

41. Wang, Z., et al., Dihydroartemisinin induces autophagy and inhibits the growth of iron-loaded human myeloid leukemia K562 cells via ROS toxicity. FEBS Open Bio, 2012. 2: p. 103-12. https://doi.org/10.1016/j.fob.2012.05.002.

42. Du, J., et al., DHA inhibits proliferation and induces ferroptosis of leukemia cells through autophagy dependent degradation of ferritin. Free Radic Biol Med, 2019. 131: p. 356-369. https://doi.org/10.1016/j.freeradbiomed.2018.12.011.

43. Chen, Y., et al., Dihydroartemisinin-induced unfolded protein response feedback attenuates ferroptosis via PERK/ATF4/HSPA5 pathway in glioma cells. J Exp Clin Cancer Res, 2019. 38(1): p. 402. https://doi.org/10.1186/s13046-019-1413-7.

44. Chen, H., et al., Dihydroartemisinin Sensitizes Human Lung Adenocarcinoma A549 Cells to Arsenic Trioxide via Apoptosis. Biol Trace Elem Res, 2017. 179(2): p. 203-212. https://doi.org/10.1007/s12011-017-0975-5. 
45. Yang, S., et al., Dihydroartemisinin increases gemcitabine therapeutic efficacy in ovarian cancer by inducing reactive oxygen species. J Cell Biochem, 2019.

120(1): p. 634-644. https://doi.org/10.1002/jcb.27421.

46. Lu, J.J., et al., Dihydroartemisinin induces apoptosis in HL-60 leukemia cells dependent of iron and 38 mitogen-activated protein kinase activation but independent of reactive oxygen species. Cancer Biol Ther, 2008. 7(7): p. 1017-23. https://doi.org/10.4161/cbt.7.7.6035.

47. Chen, H., et al., Artesunate inhibiting angiogenesis induced by human myeloma RPMI8226 cells. Int J Hematol, 2010. 92(4): p. 587-97. https://doi.org/10.1007/s12185-010-0697-3.

48. Tran, K.Q., A.S. Tin, and G.L. Firestone, Artemisinin triggers a G1 cell cycle arrest of human Ishikawa endometrial cancer cells and inhibits cyclin-dependent kinase-4 promoter activity and expression by disrupting nuclear factor-kappaB transcriptional signaling. Anticancer Drugs, 2014. 25(3): p. 270-81. https://doi.org/10.1097/CAD.0000000000000054.

49. Hirschhorn, T. and B.R. Stockwell, The development of the concept of ferroptosis. Free Radic Biol Med, 2019. 133: p. 130-143. https://doi.org/10.1016/j.freeradbiomed.2018.09.043.

50. Lin, R., et al., Dihydroartemisinin (DHA) induces ferroptosis and causes cell cycle arrest in head and neck carcinoma cells. Cancer Lett, 2016. 381(1): p. 16575. https://doi.org/10.1016/j.canlet.2016.07.033.

51. Pakos-Zebrucka, K., et al., The integrated stress response. EMBO Rep, 2016. 17(10): p. 1374-1395. https://doi.org/10.15252/embr.201642195.

52. Taniuchi, S., et al., Integrated stress response of vertebrates is regulated by four eIF2alpha kinases. Sci Rep, 2016. 6: p. 32886. https://doi.org/10.1038/srep32886.

53. Bertolotti, A., et al., Dynamic interaction of BiP and ER stress transducers in the unfolded-protein response. Nat Cell Biol, 2000. 2(6): p. 326-32. https://doi.org/10.1038/35014014.

54. Anda, S., R. Zach, and B. Grallert, Activation of Gcn2 in response to different stresses. PLoS One, 2017. 12(8): p. e0182143. https://doi.org/10.1371/journal.pone.0182143.

55. Lemaire, P.A., et al., Mechanism of PKR Activation by dsRNA. J Mol Biol, 2008. 381(2): p. 351-60. https://doi.org/10.1016/j.jmb.2008.05.056.

56. Rafie-Kolpin, M., A.P. Han, and J.J. Chen, Autophosphorylation of threonine 485 in the activation loop is essential for attaining eIF2alpha kinase activity of HRI. Biochemistry, 2003. 42(21): p. 6536-44. https://doi.org/10.1021/bi034005v.

57. Rafie-Kolpin, M., et al., Two heme-binding domains of heme-regulated eukaryotic initiation factor-2alpha kinase. $N$ terminus and kinase insertion. J Biol Chem, 2000. 275(7): p. 5171-8. https://doi.org/10.1074/jbc.275.7.5171.

58. McCormick, C. and D.A. Khaperskyy, Translation inhibition and stress granules in the antiviral immune response. Nat Rev Immunol, 2017. 17(10): p. 647-660. https://doi.org/10.1038/nri.2017.63.

59. Lee, Y.Y., R.C. Cevallos, and E. Jan, An upstream open reading frame regulates translation of GADD34 during cellular stresses that induce eIF alpha phosphorylation. J Biol Chem, 2009. 284(11): p. 6661-73. https://doi.org/10.1074/jbc.M806735200. 
60. Chan, C.P., et al., Internal ribosome entry site-mediated translational regulation of ATF4 splice variant in mammalian unfolded protein response. Biochim Biophys Acta, 2013. 1833(10): p. 2165-75. https://doi.org/10.1016/j.bbamcr.2013.05.002.

61. Han, A.P., et al., Heme-regulated eIF2alpha kinase (HRI) is required for translational regulation and survival of erythroid precursors in iron deficiency. EMBO J, 2001. 20(23): p. 6909-18. https://doi.org/10.1093/emboj/20.23.6909.

62. Novoa, I., et al., Feedback inhibition of the unfolded protein response by GADD34-mediated dephosphorylation of eIF2alpha. J Cell Biol, 2001. 153(5): p. 1011-22. https://doi.org/10.1083/jcb.153.5.1011.

63. Puthalakath, H., et al., ER stress triggers apoptosis by activating BH3-only protein Bim. Cell, 2007. 129(7): p. 1337-49. https://doi.org/10.1016/j.cell.2007.04.027.

64. Jin, S., et al., 5-Azacitidine Induces NOXA to Prime AML Cells for VenetoclaxMediated Apoptosis. Clin Cancer Res, 2020. 26(13): p. 3371-3383. https://doi.org/10.1158/1078-0432.CCR-19-1900.

65. Pike, L.R., et al., ATF4 orchestrates a program of BH3-only protein expression in severe hypoxia. Mol Biol Rep, 2012. 39(12): p. 10811-22. https://doi.org/10.1007/s11033-012-1975-3.

66. Jabbour, E., et al., New insights into the pathophysiology and therapy of adult acute lymphoblastic leukemia. Cancer, 2015. 121(15): p. 2517-28. https://doi.org/10.1002/cncr.29383.

67. Institute, N.N.C., Cancer Stat Facts: Leukemia - Acute Lymphocytic Leukemia (ALL).

68. Mullighan, C.G., How advanced are we in targeting novel subtypes of ALL? Best Pract Res Clin Haematol, 2019. 32(4): p. 101095. https://doi.org/10.1016/j.beha.2019.101095.

69. Koss, B., et al., Requirement for antiapoptotic MCL-1 in the survival of BCR-ABL B-lineage acute lymphoblastic leukemia. Blood, 2013. 122(9): p. 1587-98. https://doi.org/10.1182/blood-2012-06-440230.

70. Clark, S.S., et al., Unique forms of the abl tyrosine kinase distinguish Ph1-positive CML from Ph1-positive ALL. Science, 1987. 235(4784): p. 85-8. https://doi.org/10.1126/science.3541203.

71. Abou Dalle, I., et al., Philadelphia chromosome-positive acute lymphoblastic leukemia at first relapse in the era of tyrosine kinase inhibitors. Am J Hematol, 2019. 94(12): p. 1388-1395. https://doi.org/10.1002/ajh.25648.

72. Cilloni, D. and G. Saglio, Molecular pathways: BCR-ABL. Clin Cancer Res, 2012. 18(4): p. 930-7. https://doi.org/10.1158/1078-0432.CCR-10-1613.

73. Roberts, K.G., Why and how to treat Ph-like ALL? Best Pract Res Clin Haematol, 2018. 31(4): p. 351-356. https://doi.org/10.1016/j.beha.2018.09.003.

74. Roberts, K.G., et al., High Frequency and Poor Outcome of Philadelphia Chromosome-Like Acute Lymphoblastic Leukemia in Adults. J Clin Oncol, 2017. 35(4): p. 394-401. https://doi.org/10.1200/JCO.2016.69.0073.

75. Nicolini, F.E., et al., Epidemiologic study on survival of chronic myeloid leukemia and Ph(+) acute lymphoblastic leukemia patients with BCR-ABL T315I mutation. Blood, 2009. 114(26): p. 5271-8. https://doi.org/10.1182/blood-2009-04-219410. 
76. Zhang, Y., et al., PDGFRB mutation and tyrosine kinase inhibitor resistance in Ph-like acute lymphoblastic leukemia. Blood, 2018. 131(20): p. 2256-2261. https://doi.org/10.1182/blood-2017-11-817510.

77. Smith, K.H., et al., The Heme-Regulated Inhibitor Pathway Modulates Susceptibility of Poor Prognosis B-Lineage Acute Leukemia to BH3-Mimetics. Mol Cancer Res, 2020. https://doi.org/10.1158/1541-7786.MCR-20-0586.

78. $\quad$ Roberts, K.G., et al., Targetable kinase-activating lesions in Ph-like acute lymphoblastic leukemia. N Engl J Med, 2014. 371(11): p. 1005-15. https://doi.org/10.1056/NEJMoa1403088.

79. Doench, J.G., et al., Optimized sgRNA design to maximize activity and minimize off-target effects of CRISPR-Cas9. Nat Biotechnol, 2016. 34(2): p. 184-191. https://doi.org/10.1038/nbt.3437.

80. Li, W., et al., Quality control, modeling, and visualization of CRISPR screens with MAGeCK-VISPR. Genome Biol, 2015. 16: p. 281. https://doi.org/10.1186/s13059-015-0843-6.

81. Chen, E.Y., et al., Enrichr: interactive and collaborative HTML5 gene list enrichment analysis tool. BMC Bioinformatics, 2013. 14: p. 128. https://doi.org/10.1186/1471-2105-14-128.

82. Kuleshov, M.V., et al., Enrichr: a comprehensive gene set enrichment analysis web server 2016 update. Nucleic Acids Res, 2016. 44(W1): p. W90-7. https://doi.org/10.1093/nar/gkw377.

83. Connelly, J.P. and S.M. Pruett-Miller, CRIS.py: A Versatile and High-throughput Analysis Program for CRISPR-based Genome Editing. Sci Rep, 2019. 9(1): p. 4194. https://doi.org/10.1038/s41598-019-40896-w.

84. Stewart, D.P., et al., Ubiquitin-independent degradation of antiapoptotic MCL-1. Mol Cell Biol, 2010. 30(12): p. 3099-110. https://doi.org/10.1128/MCB.01266$\underline{09}$.

85. Fukuda, Y., et al., Upregulated heme biosynthesis, an exploitable vulnerability in MYCN-driven leukemogenesis. JCI Insight, 2017. 2(15). https://doi.org/10.1172/jci.insight.92409.

86. Greco, W.R., H.S. Park, and Y.M. Rustum, Application of a new approach for the quantitation of drug synergism to the combination of cis-

diamminedichloroplatinum and 1-beta-D-arabinofuranosylcytosine. Cancer Res, 1990. 50(17): p. 5318-27.

87. Minto, C.F., et al., Response surface model for anesthetic drug interactions. Anesthesiology, 2000. 92(6): p. 1603-16. https://doi.org/10.1097/00000542$200006000-00017$.

88. Jonker, D.M., et al., Towards a mechanism-based analysis of pharmacodynamic drug-drug interactions in vivo. Pharmacol Ther, 2005. 106(1): p. 1-18. https://doi.org/10.1016/j.pharmthera.2004.10.014.

89. Churchman, M.L., et al., Efficacy of Retinoids in IKZF1-Mutated BCR-ABLI Acute Lymphoblastic Leukemia. Cancer Cell, 2015. 28(3): p. 343-56. https://doi.org/10.1016/j.ccell.2015.07.016.

90. Notta, F., et al., Evolution of human BCR-ABL1 lymphoblastic leukaemiainitiating cells. Nature, 2011. 469(7330): p. 362-7. https://doi.org/10.1038/nature09733. 
91. Rowe, J.M., et al., Induction therapy for adults with acute lymphoblastic leukemia: results of more than 1500 patients from the international ALL trial: MRC UKALL XII/ECOG E2993. Blood, 2005. 106(12): p. 3760-7. https://doi.org/10.1182/blood-2005-04-1623.

92. Tse, C., et al., ABT-263: a potent and orally bioavailable Bcl-2 family inhibitor. Cancer Res, 2008. 68(9): p. 3421-8. https://doi.org/10.1158/0008-5472.CAN-075836.

93. Chiabrando, D., et al., Heme in pathophysiology: a matter of scavenging, metabolism and trafficking across cell membranes. Front Pharmacol, 2014. 5: p. 61. https://doi.org/10.3389/fphar.2014.00061.

94. Baird, T.D. and R.C. Wek, Eukaryotic initiation factor 2 phosphorylation and translational control in metabolism. Adv Nutr, 2012. 3(3): p. 307-21. https://doi.org/10.3945/an.112.002113.

95. Crosby, J.S., et al., Regulation of hemoglobin synthesis and proliferation of differentiating erythroid cells by heme-regulated eIF-2alpha kinase. Blood, 2000. 96(9): p. 3241-8.

96. Sidrauski, C., et al., The small molecule ISRIB reverses the effects of eIF2alpha phosphorylation on translation and stress granule assembly. Elife, 2015. 4. https://doi.org/10.7554/eLife.05033.

97. Chen, T., et al., Chemical genetics identify eIF2alpha kinase heme-regulated inhibitor as an anticancer target. Nat Chem Biol, 2011. 7(9): p. 610-6. https://doi.org/10.1038/nchembio.613.

98. Lam, M., et al., Evidence that BCL-2 represses apoptosis by regulating endoplasmic reticulum-associated Ca2+ fluxes. Proc Natl Acad Sci U S A, 1994. 91(14): p. 6569-73. https://doi.org/10.1073/pnas.91.14.6569.

99. Brennan, M.S., et al., Humanized Mcl-1 mice enable accurate preclinical evaluation of MCL-1 inhibitors destined for clinical use. Blood, 2018. 132(15): p. 1573-1583. https://doi.org/10.1182/blood-2018-06-859405.

100. Hooda, J., et al., Enhanced heme function and mitochondrial respiration promote the progression of lung cancer cells. PLoS One, 2013. 8(5): p. e63402. https://doi.org/10.1371/journal.pone.0063402.

101. Zhang, Q., et al., New activators of eIF2alpha Kinase Heme-Regulated Inhibitor (HRI) with improved biophysical properties. Eur J Med Chem, 2020. 187: p. 111973. https://doi.org/10.1016/j.ejmech.2019.111973.

102. Lin, K.H., et al., Systematic Dissection of the Metabolic-Apoptotic Interface in AML Reveals Heme Biosynthesis to Be a Regulator of Drug Sensitivity. Cell Metab, 2019. 29(5): p. 1217-1231 e7. https://doi.org/10.1016/j.cmet.2019.01.011.

103. Gomez-Bougie, P., et al., Repression of Mcl-1 and disruption of the Mcl-1/Bak interaction in myeloma cells couple ER stress to mitochondrial apoptosis. Cancer Lett, 2016. 383(2): p. 204-211. https://doi.org/10.1016/j.canlet.2016.09.030.

104. Ganz, J., et al., A novel specific PERK activator reduces toxicity and extends survival in Huntington's disease models. Sci Rep, 2020. 10(1): p. 6875. https://doi.org/10.1038/s41598-020-63899-4.

105. Li, X., et al., CCT020312 Inhibits Triple-Negative Breast Cancer Through PERK Pathway-Mediated G1 Phase Cell Cycle Arrest and Apoptosis. Front Pharmacol, 2020. 11: p. 737. https://doi.org/10.3389/fphar.2020.00737. 
106. Punnoose, E.A., et al., Expression Profile of BCL-2, BCL-XL, and MCL-1

Predicts Pharmacological Response to the BCL-2 Selective Antagonist Venetoclax in Multiple Myeloma Models. Mol Cancer Ther, 2016. 15(5): p. 113244. https://doi.org/10.1158/1535-7163.MCT-15-0730.

107. Li, Z., S. He, and A.T. Look, The MCL1-specific inhibitor S63845 acts synergistically with venetoclax/ABT-199 to induce apoptosis in T-cell acute lymphoblastic leukemia cells. Leukemia, 2019. 33(1): p. 262-266. https://doi.org/10.1038/s41375-018-0201-2.

108. Burwick, N., et al., The eIF2-alpha kinase HRI is a novel therapeutic target in multiple myeloma. Leuk Res, 2017. 55: p. 23-32. https://doi.org/10.1016/j.leukres.2017.01.007.

109. Lee, K.M., et al., MYC and MCL1 Cooperatively Promote ChemotherapyResistant Breast Cancer Stem Cells via Regulation of Mitochondrial Oxidative Phosphorylation. Cell Metab, 2017. 26(4): p. 633-647 e7. https://doi.org/10.1016/j.cmet.2017.09.009.

110. Merino, D., et al., Synergistic action of the MCL-1 inhibitor S63845 with current therapies in preclinical models of triple-negative and HER2-amplified breast cancer. Sci Transl Med, 2017. 9(401). https://doi.org/10.1126/scitranslmed.aam7049.

111. Shen, J., et al., Iron metabolism regulates p53 signaling through direct heme-p53 interaction and modulation of p53 localization, stability, and function. Cell Rep, 2014. 7(1): p. 180-93. https://doi.org/10.1016/j.celrep.2014.02.042.

112. Zhou, M., et al., Targeting of the deubiquitinase USP9X attenuates B-cell acute lymphoblastic leukemia cell survival and overcomes glucocorticoid resistance. Biochem Biophys Res Commun, 2015. 459(2): p. 333-339. https://doi.org/10.1016/j.bbrc.2015.02.115. 


\section{VITA}

Kaitlyn Smith was born in Gastonia, NC in 1994. She completed a Bachelor of Science degree in biochemistry from Mississippi State University in May of 2016. She enrolled in the Biomedical Sciences Ph.D. program at the University of Tennessee Health Science Center in 2016 and joined the Opferman laboratory at St. Jude in 2017. On April $1^{\text {st }}, 2017$ she married Chase Smith, and they welcomed their first child, Liam Smith, in December of 2020. She is expected to graduate in March 2021.

\section{Publications}

1. Meghan E. Turnis, Ewa Kaminska, Kaitlyn H. Smith, Brittany J. Kartchner, Peter Vogel, Jonathan D. Laxton, Richard A. Ashmun, Paul A. Ney, Joseph T. Opferman. Requirement for Anti-Apoptotic MCL-1 during Early Erythropoiesis. Blood. 2021.

2. Kaitlyn H Smith*, Amit Budhraja*, John Lynch, Kathryn Roberts, John C Panetta, Jon P Connelly, Meghan E Turnis, Shondra M Pruett-Miller, John D Schuetz, Charles G Mullighan, Joseph T Opferman. The Heme-Regulated Inhibitor Pathway Modulates Susceptibility of Poor Prognosis B-Lineage Acute Leukemia to BH3Mimetics. Molecular Cancer Research. 2020. *equal contribution

3. Timothy B Johnstone, Kaitlyn H Smith, Cynthia J Koziol-White, Fengying Li, Austin G Kazarian, Maia L Corpuz, Maya Shumyatcher, Frederick J Ehlert, Blanca E Himes, Reynold A Panettieri Jr, Rennolds S Ostrom. PDE8 Is Expressed in Human Airway Smooth Muscle and Selectively Regulates cAMP Signaling by $\beta_{2}$-Adrenergic Receptors and Adenylyl Cyclase 6. American Journal of Respiratory Cell and Molecular Biology. 2017. 